\title{
Climatic and Ecohydrologic Linkages of Net Ecosystem Exchanges (NEE) across Diverse Ecosystems
}

Mohammed Tamim Zaki

Follow this and additional works at: https://researchrepository.wvu.edu/etd

\section{Recommended Citation}

Zaki, Mohammed Tamim, "Climatic and Ecohydrologic Linkages of Net Ecosystem Exchanges (NEE) across Diverse Ecosystems" (2017). Graduate Theses, Dissertations, and Problem Reports. 7022. https://researchrepository.wvu.edu/etd/7022

This Thesis is protected by copyright and/or related rights. It has been brought to you by the The Research Repository @ WVU with permission from the rights-holder(s). You are free to use this Thesis in any way that is permitted by the copyright and related rights legislation that applies to your use. For other uses you must obtain permission from the rights-holder(s) directly, unless additional rights are indicated by a Creative Commons license in the record and/ or on the work itself. This Thesis has been accepted for inclusion in WVU Graduate Theses, Dissertations, and Problem Reports collection by an authorized administrator of The Research Repository @ WVU. For more information, please contact researchrepository@mail.wvu.edu. 


\title{
Climatic and Ecohydrologic Linkages of Net Ecosystem Exchanges (NEE) across Diverse Ecosystems
}

\author{
Mohammed Tamim Zaki
}

\author{
Thesis submitted to the \\ Benjamin M. Statler College of Engineering and Mineral Resources at \\ West Virginia University
}

in partial fulfillment of the requirements for the degree of

Master of Science in

Civil Engineering

\author{
Omar I. Abdul-Aziz, Ph.D., Chair \\ Kakan Dey, Ph.D. \\ Khandker S. Ishtiaq, Ph.D.
}

Department of Civil and Environmental Engineering

\author{
Morgantown, West Virginia \\ 2017
}

Keywords:

Net ecosystem exchange, climatic-ecohydrologic drivers, data analytics, diverse ecosystems, multi-temporal linkages

Copyright 2017, Mohammed Tamim Zaki 


\section{ABSTRACT \\ Climatic and Ecohydrologic Linkages of Net Ecosystem Exchanges (NEE) across Diverse Ecosystems}

Mohammed Tamim Zaki

Net ecosystem exchange (NEE) indicates the balance between photosynthetic uptake and respiration of $\mathrm{CO}_{2}$ in a biological system. A large set of climatic and ecohydrological variables drives the dynamics of NEE through complex, interacting biophysical processes. The relative climatic and ecohydrologic linkages of NEE were determined in this study for different temporal scales (i.e., 1-hour, 1-day, 8-day, 15-day, and 30-day) by utilizing a systematic data analytics methodology. Pearson correlation matrix and factor analysis were employed to identify the interrelations and grouping patterns among the biophysical variables. The climatic and ecohydrologic linkages of NEE were then estimated using bootstrapped (1000 iterations) partial least squares regression by appropriately resolving multicollinearity among the drivers. Four biophysical components of NEE were identified based on hourly datasets from 57 AmeriFlux sites, representing six diverse ecosystems (wetland, cropland, grassland, evergreen, deciduous, and mixed forests) across North America. The multi-temporal (1-day to 30-day) dataset for the Florida Everglades short-hydroperiod marsh also indicated similar groupings. In general, NEE was strongly linked with the 'radiation-energy' (RE) component, while having a moderate linkage with the 'temperature-hydrology' (TH) component across the temporal scales. The 'aerodynamic' (AD) component was weakly linked to NEE; however, a moderate AD control on NEE was observed in the short canopy ecosystems at the 1-hour scale. The 'atmospheric $\mathrm{CO}_{2}$ ' (AC) component exhibited moderate to weak linkages with NEE across the temporal scales. RE and TH had a decreasing trend with the increasing time-scales (1-30 day) at the Everglades short-hydroperiod site. In contrast, the linkages of AD and AC components increased from 1-day to 8-day scales, and then remained relatively unchanged at the longer scales of aggregation. The estimated linkages provided valuable insights into the biophysical process components and drivers of ecosystem carbon across variable spatial and temporal scales. The findings would also help to develop robust, low-dimensional models to reliably predict ecosystem $\mathrm{CO}_{2}$ fluxes at variable times and space scales. 


\section{ACKNOWLEDGEMENTS}

I would like to express my sincere gratitude to my supervisor, Dr. Omar I. Abdul-Aziz, for his continuous and valuable guidance, counselling and inspiration throughout my research work. Without his active interest, it would have been difficult for me to fulfill the objectives of my research. I would like to thank Dr. Kakan Dey and Dr. Khandker S. Ishtiaq for being my defense committee members and providing me with their utmost advice, support and encouragement.

I would genuinely like to thank all of my lab-mates and their families for their support and guidance to fuel me with courage during challenging times faced throughout my academic and research work.

I would like to gratefully acknowledge funding awarded to Dr. Omar I. Abdul-Aziz from National Science Foundation (NSF) CBET Environmental Sustainability (Award No. 1705941), National Oceanic and Atmospheric Administration (NOAA) (Grant No. NA14NOS4190145) and WVU Faculty Start-up funding.

Lastly, and most importantly I would like to dedicate my thesis to my parents for instilling in me the importance of education all through my life and my sisters for their limitless support. 


\section{Table of Contents}

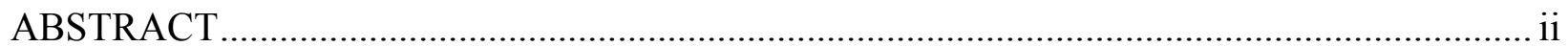

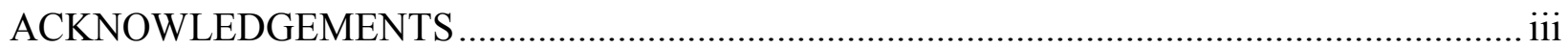

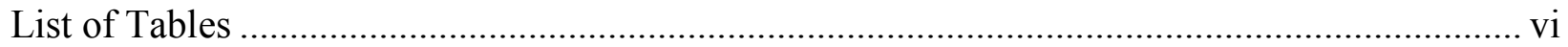

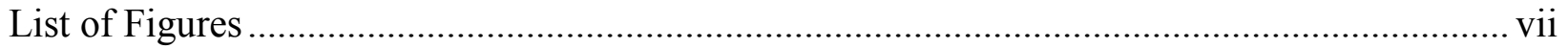

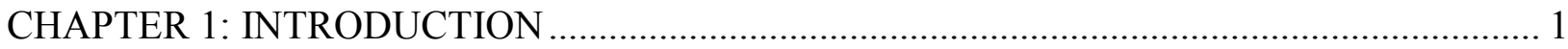

1.1 Background and motivation ........................................................................................ 1

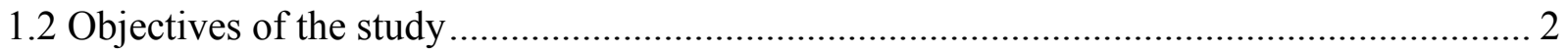

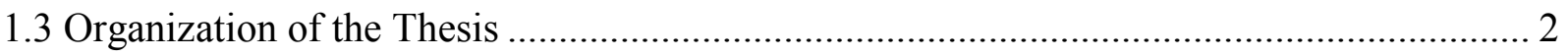

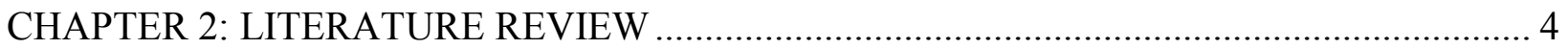

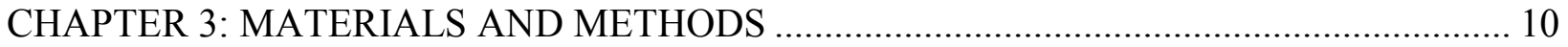

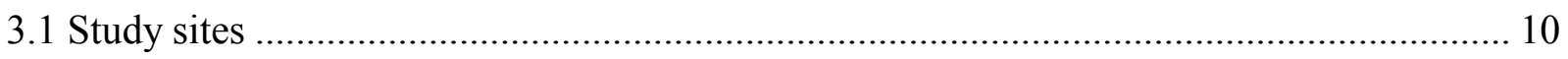

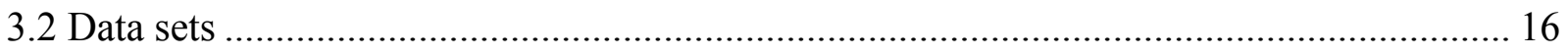

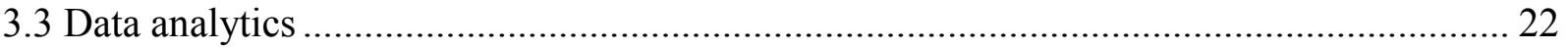

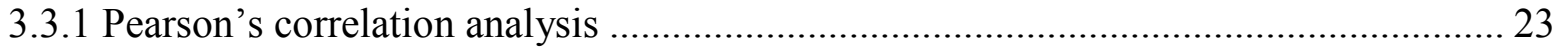

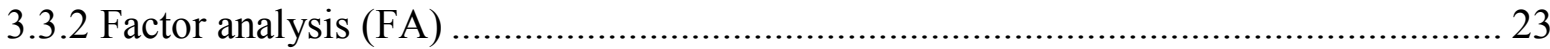

3.3.3 Partial least squares regression (PLSR) modeling....................................................... 24

CHAPTER 4: RESULTS OF HOURLY LINKAGES OF NEE ACROSS DIVERSE

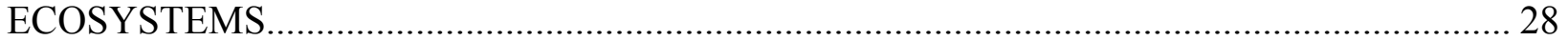

4.1 Linear relationships from correlation analysis ............................................................ 28

4.2 Multivariate interrelations and grouping patterns from FA ………................................. 31

4.3 Formation of bio-physical process components ............................................................. 34

4.4 Estimation of the linkages across diverse ecosystems .................................................... 35

CHAPTER 5: RESULTS OF MULTI-TEMPORAL LINKAGES OF NEE AT THE

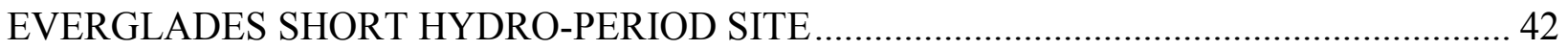

5.1 Linear relationships from correlation analysis ................................................................. 42

5.2 Multivariate interrelations and grouping patterns from FA ………………………......... 44

5.3 Formation of bio-physical process components........................................................... 47

5.4 Estimation of the multi-temporal linkages at the Florida Everglades short hydro-period .. 47 


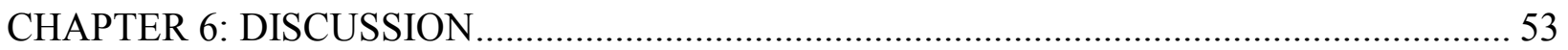

6.1 On the hourly linkages across diverse ecosystems..................................................... 53

6.2 On the multi-temporal linkages at the Everglades short hydro-period site....................... 55

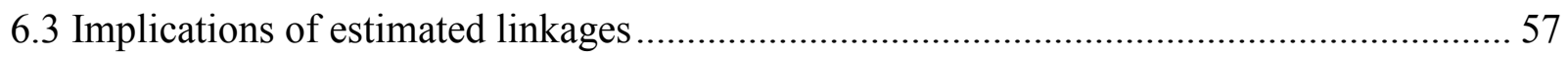

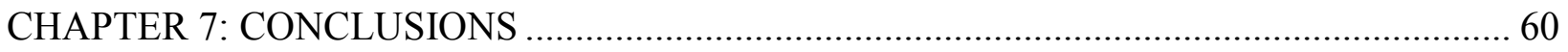

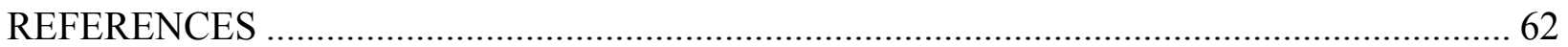

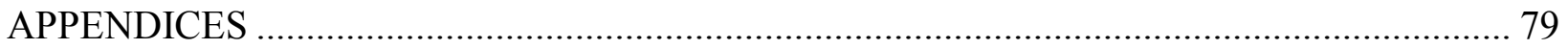




\section{List of Tables}

Table 3.1: Biophysical characteristics of the 57 AmeriFlux study sites representing six diverse ecosystem.

Table 3.2: Data summary of observed hourly NEE, climatic, and ecohydrological variables by ecosystem type.

Table 3.3: Data summary of NEE, climatic, and ecohydrological variables at the different temporal scales for the Florida Everglades short hydro-period marsh site.

Table 4.1: Mean bivariate Pearson's correlation coefficients $(r)$ between NEE and the climatic and ecohydrological variables across diverse ecosystems.

Table 4.2: Dominant hidden factors and ensemble mean loadings of the participatory variables across the diverse ecosystems.

Table 4.3: Ensemble means and standard deviations (in parenthesis) of the Z-score PLSR model coefficients $(\beta)$, representing the relative linkages of NEE under different ecosystem types. The ecosystem type-specific statistics were computed from the corresponding site-specific $\beta$, as obtained from 1000 Monte-Carlo estimations.

Table 5.1: Pearson's correlation coefficients $(r)$ between NEE and climatic and ecohydrological variables at different temporal scales.

Table 5.2: Percent variance explained by each factor at the different temporal scales for the Florida Everglades short hydro-period site.

Table 5.3: Dominant latent factors and optimized loadings of NEE, climatic, and ecohydrological variables at different temporal scales.

Table 5.4: Z-score PLSR model parameters $(\beta)$ of NEE and standard deviations (in parenthesis) generated from 1000 Monte-Carlo estimations at the four temporal scales. 50 


\section{List of Figures}

Figure 3.1: Locations of the 57 AmeriFlux study sites representing six diverse ecosystem........ 10

Figure 3.2: Location of the case study site at Florida everglades short hydro-period marsh. ...... 16

Figure 3.3: Stepwise representation of the data analytics approach to determine the climatic and

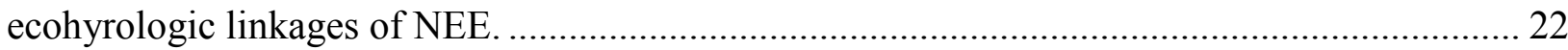

Figure 4.1: Mean correlation coefficients among NEE and climatic and ecohydrological variables for the diverse ecosystem represented in a triangular map.

Figure 4.2: Percent variance explained by each factor for the six diverse ecosystems comprising 57 sites at the hourly scale.

Figure 4.3: Factor loadings of the participatory variables across the 57 study sites for the first three common factors summarized.

Figure 4.4: Boxplots of cross-validated (a) NSE of NEE, (b) normalized AIC, and (c) eigenvalues of the data matrix with the number of partial least squares (PLS) components across the 57 sites

Figure 4.5: Summary of the estimated PLSR model coefficients $(\beta)$ for the diverse ecosystems.37

Figure 4.6: Aggregated linkages of NEE with the major biophysical process components across different ecosystems. ENF, DBF, MF, CRO, GRA, and WET refer to evergreen needleleaf forests, deciduous broadleaf forests, mixed forests, croplands, grasslands, and wetlands, respectively. Error bars represent 95\% upper and lower confidence limits of the aggregated linkages.

Figure 5.1: Triangular map representing correlation coefficients of NEE, climatic, and ecohydrological variables for the different temporal scales.

Figure 5.2: Observed vs. predicted Z-scores of NEE at different temporal scales. NEE was predicted using the Z-score PLSR model parameters $(\beta)$ obtained from 1000 Monte-Carlo estimations. The dashed line represents the 1:1 line.

Figure 5.3: Aggregated linkages of NEE with the major biophysical components at different temporal scales. Error bars represent $95 \%$ confidence limits of the aggregated linkages. 


\section{CHAPTER 1: INTRODUCTION}

\subsection{Background and motivation}

The land-atmospheric exchanges of carbon dioxide $\left(\mathrm{CO}_{2}\right)$ substantially contributes to the terrestrial carbon balance (Stoy et al., 2009, Shi et al., 2017). The net ecosystem exchange (NEE) of $\mathrm{CO}_{2}$ is an important indicator of the carbon balance representing the difference between carbon assimilated in terrestrial ecosystems as a result of photosynthetic fixation and carbon released into the atmosphere by ecosystem respiratory processes (autotrophic and heterotrophic respiration) (Law et al., 2002, Heskel et al., 2013, Baldocchi et al., 2017). The variation of NEE is largely sensitive to the variations of climatic and ecohydrological variables across space and time (Stoy et al., 2005, Baldocchi et al., 2017). The linkages between NEE and its climatic and ecohydrological drivers can provide information on the sensitivities of land carbon sink and source. Furthermore, proper representation of these linkages is crucial to understand the complex interactions between the carbon cycle and the climate system (Fang and Michalak, 2015). Previous studies identified the climatic and ecohydrologic influences of NEE at different spatial and temporal scales (i.e., Law et al., 2002, Xiao et al., 2008, Schmidt et al., 2011, Xiao et al., 2014). However, the critical question of whether these influences are similar across spatiotemporal scales is yet to be answered. The spatiotemporal similarity of the linkages would imply the requirement of minimum sitespecific calibration, therefore, would provide guidance toward the development of spatiotemporally robust $\mathrm{CO}_{2}$ flux models. The study aimed at investigating the spatiotemporal similarity of the climatic and ecohyrological linkages of NEE. A systematic data analytics methodology comprising of Pearson's correlation analysis, factor analysis (FA), and partial least squares regression (PLSR) was employed to determine these linkages and explore their spatiotemporal similarities. Correlation analysis and FA are pattern recognition techniques that 
can detect hidden information in large datasets and help identify meaningful linkage patterns. Moreover, PLSR quantifies the true linkages of the variables that drive NEE through resolving multicollinearity. Therefore, the entire data analytics methodology provide an appropriate representation of the climatic and ecohydrologic linkages of NEE across spatiotemporal scales.

\subsection{Objectives of the study}

The primary objective of the study was to investigate the similarity-based linkages of NEE with the climatic and ecohydrological drivers across spatiotemporal scales. The Pearson's correlation, FA, and PLSR were sequentially utilized to identify the interrelations, grouping patterns, and linkages of the climatic and ecohydrological variables with NEE.

The specific objectives of the study are as follows:

- Investigate the climatic and ecohydrologic linkages of NEE across six diverse ecosystems of continental North America at the 1-hour scale.

- Investigate the climatic and ecohydrologic linkages of NEE from 1-day to 30-day scale for the Florida Everglades short hydro-period marsh site.

\subsection{Organization of the Thesis}

The thesis is composed of seven chapters. The current chapter delivers a brief introduction and motivation of the study along with the research objectives, and the organization of the thesis. Chapter 2 provides a detailed literature review of the studies conducted to identify climatic and environmental/ecohydrological controls of NEE. Chapter 3 describes the study sites, data sets, and detailed methodology involved in the research. Chapter 4 reports the results obtained from the hourly linkage analysis of NEE across diverse ecosystems. Chapter 5 provides the results attained from the multi-temporal linkage analysis of NEE at the Everglades short hydro-period marsh site. 
Chapter 6 comprises of detailed discussion on the linkages estimated at the hourly scale across diverse ecosystems and the multi-temporal linkages attained for the case study site. This chapter also includes the implications of the quantified linkages about understanding the functions of development of predictive models and ecosystem metabolism. Chapter 7 is the conclusions where significant findings from the research were summarized. 


\section{CHAPTER 2: LITERATURE REVIEW}

The variation of NEE is regulated by two biological processes (photosynthesis and respiration), exhibiting dynamic variations across ecosystems in response to the effects of various climatic and ecohydrological drivers (Stoy et al., 2009, Wagle et al., 2017). The development of comprehensive records of carbon, energy, and water fluxes along with their concurrent climatic and ecohydrological observations collected from numerous eddy-covariance flux towers under the AmeriFlux network has created prospects for scientists and researchers to investigate the intricate interactions between the biosphere and the atmosphere (Baldocchi et al., 2001). By utilizing this enormous data repository, many studies have been conducted over the past few decades to identify the roles of important climatic and ecohydrological/environmental drivers that drive ecosystem fluxes of $\mathrm{CO}_{2}$.

The environmental drivers of NEE at a Central American tropical wet forest located in the Caribbean lowlands of Costa Rica were studied by Loescher et al. (2003). Using observations from 1998 to 2000, the study showed moderate to strong relationships of daytime NEE with photosynthetically active radiation, air temperature, and vapor pressure deficit for the tropical wet forest. Huxman et al. (2003) investigated the control of air temperature on NEE in a high elevation evergreen needleleaf forest using path analysis evaluation that was similar to multiple regressions analysis. The growing season data from 1999 and 2000 of the Niwot ridge forest showed that NEE was strongly linked with relatively low air temperature during the growing season. A study by Schedlbauer et al. (2010) in the sites of Florida Everglades showed that photosynthetically active radiation and air temperature responded to NEE significantly differently in variable seasons. The study also revealed the importance of water level as a significant driver of NEE in those sites. 
Another study conducted by Barr et al. (2010) reported that NEE variability was controlled by photosynthetically active radiation along with air temperature and further suggested that tidal activity was also a significant driver for wetland ecosystem $\mathrm{CO}_{2}$ flux. Schedlbauer et al. (2012) showed that air temperature and vapor pressure deficit were important drivers of NEE in the Everglades short-hydroperiod marsh. The importance of water level as a driver of NEE in wetland sites was reflected by a study conducted by Jimenez et al. (2012). The study further showed significant effects of photosynthetically active radiation, air temperature, and vapor pressure deficit on NEE.

Large-scale studies focused on determining the key drivers that influence terrestrial $\mathrm{CO}_{2}$ fluxes using multiple sites representing different vegetation types. Law et al. (2002) investigated the variations of $\mathrm{CO}_{2}$ exchange in response to climatic and environmental drivers across 35 sites of evergreen needleleaf, evergreen broadleaf, deciduous broadleaf and mixed forests as well as grasslands, tundra, and croplands all over North America and Europe using observational data for the period of 1992-1999. The study explored the control of photosynthetically active radiation at cloudy and clear conditions across different ecosystems. Photosynthetically active radiation exhibited strong linkages with $\mathrm{CO}_{2}$ exchange for the ecosystems under consideration whereas air temperature showed comparatively moderate linkages. By combining 8-day resolution MODIS (Moderate Resolution Imaging Spectroradiometer) and NEE data during the period of 2000-2006 for 42 AmeriFlux sites across evergreen, deciduous, mixed forests, grasslands, croplands, and savannas of the conterminous United States, Xiao et al. (2008) developed a predictive model of NEE using the modified regression tree approach. Explanatory variables from MODIS used in this model were land surface temperature (LST), enhanced vegetation index (EVI), and fraction of photosynthetically active radiation (fPAR). Another study conducted by Schmidt et al. (2011) 
identified major environmental and meteorological drivers influencing $\mathrm{CO}_{2}$ flux from different ecosystem types consisting of 56 AmeriFlux sites. Applying a self-organizing feature map (SOFM) neural network procedure on daily averaged data during 1992-2009, the study revealed net radiation and photosynthetically active radiation as the most important drivers of ecosystem $\mathrm{CO}_{2}$ flux followed by vegetation type. The importance of vapor pressure deficit, soil temperature, precipitation, and wind speed followed next. Xiao et al. (2014) developed site level predictive models of $\mathrm{CO}_{2}$ fluxes for 94 sites across the United States and Canada, where the explanatory variables involved photosynthetically active radiation, LST, EVI, normalized difference water index (NDWI), stand age, and aboveground biomass. An atmospheric-inversion-based framework was applied by Fang and Michalak (2015) for four different ecosystems across North America to identify relationships of NEE with its enviroclimatic drivers. The study found that shortwave radiation was the strongest driver in regulating NEE. Specific humidity and precipitation also played key roles in driving NEE. A comprehensive review of published $\mathrm{CO}_{2}$ flux modeling studies was conducted by Baldocchi et al. (2017). The study summarized that a set of climatic and ecohydrological factors, such as, light, temperature, rainfall, phenology plays important roles in driving the variability of net ecosystem $\mathrm{CO}_{2}$ fluxes.

It is evident that current literature on ecosystem $\mathrm{CO}_{2}$ flux studies focuses on determining the linkages of important climatic and environmental/ecohydrological drivers of NEE, and ounfolding their complex interactions and underlying biological processes. These identified linkages are very important to indicate the major biophysical process variables of NEE to better inform the existing earth-system models. However, an important question that remained unanswered with the availability of rich databases (i.e., AmeriFlux, Fluxnet 2015) of terrestrial $\mathrm{CO}_{2}$ fluxes and its concurrent climatic and ecohydrological variables across diverse ecosystem 
types and large periods of time is that whether these linkages are similar over spatiotemporal scales. The spatiotemporal similarity of linkages would simply indicate that the model parameters calibrated for a particular site would be transferable to other sites. Therefore, large-scale sitespecific calibration would be minimized that would lead to the development of spatiotemporally robust ecosystem $\mathrm{CO}_{2}$ flux models. Ishtiaq and Abdul-Aziz (2015), showed similar climatic and environmental linkages of NEE for multiple sites under eight deciduous forests of the United States. This study builds on the results of the aforementioned paper to investigate that these linkages are similar across spatial scale comprising six diverse ecosystem types of the continental North America.

The investigation of climatic and ecohydrologic linkages of NEE across temporal scales is a relatively unexplored territory compared to the linkages across spatial scales. A wetland ecosystem site, Florida Everglades short hydro-period marsh was selected as a case study site to explore the multi-temporal climatic and ecohydrologic linkages of NEE in this study. This site is considered as one of the most dynamic wetlands under the AmeriFlux network, as it is characterized by shallow-water sheet flow, annual recurrences of wet and dry seasons, occasional occurrences of prolonged droughts and floods as well as seasonal hurricanes and frosts (Miao and Sklar, 1997). Furthermore, the anoxic wet conditions in this site offer optimum natural environment that aid in the higher sequestration of carbon (Mitsch et al., 2013, Morin et al., 2017). An in-depth understanding of the drivers of NEE at the multi-temporal scales in such a dynamic site would certainly help develop knowledge on the characteristics of the linkages for the sites under the other ecosystems.

Data-driven modeling approaches have increasingly been used in the ecological and environmental community because of its highly desired property to combine variable selection and 
data mining tool with interpretable model results (Wylie et al., 2007). Data-driven approaches are simple, involving empirical and statistical techniques that are trained by observations of fluxes and their predictor variables (Xiao et al., 2012). Process-based modeling on the other hand, involves complex structures combined with hypotheses of intricate manner and requires high computational resources at the same time (Ishtiaq and Abdul-Aziz, 2015). Appropriate application of data-driven models can effectively capture the true relationships that exists in a data matrix. There are certain limitations to data-driven modeling. The developed models might be overfitted due to capturing noise and would not state meaningful relationships. Proper implementation of data filtering techniques can minimize such limitations. A number of recent studies have utilized the AmeriFlux network database to establish enhanced understanding of the exchanges of carbon, water, and energy fluxes using various data-driven models (i.e., Xiao et al., 2008, Jung et al., 2011, Stoy et al., 2013, Xiao et al., 2014). These models were calibrated with site-specific flux observations but the similarity of these parameters across different sites are yet to be explored.

To determine a general set of parameters for robustly modeling ecosystem $\mathrm{CO}_{2}$ flux across a diverse set of ecosystems, an appropriate framework comprised of data-driven techniques and modeling approaches is necessary. Correlation analysis is suitable for investigating bivariate linear relationships between a variable and its drivers as well as understanding the multicollinear structure of the dataset. Factor analysis (FA) on the other hand, is a more fitting technique to identify multivariate relationships among the participatory variables taking care of the multicollinearity among the variables (Ryan et al., 2015). Partial least squares regression (PLSR) modeling as a data-driven modeling approach, has been an effective tool to extract information from observational data and systematically develop quantitative linkages of the driver variables with their predictors. Matthes et al. (2015) applied the PLSR modeling technique with the 
hyperspectral canopy reflectance data set to quantify the responses of NEE at half-hourly to monthly temporal scales. Therefore, multivariate data analytics approach combining pattern recognition techniques (i.e., correlation analysis, FA etc.) and empirical modeling (i.e., PLSR) is a useful tool to process large and complex datasets of ecosystem $\mathrm{CO}_{2}$ fluxes and its drivers to appropriately quantify their linkages. Data analytics have been successfully applied in the field of ecological and environmental sciences. Ishtiaq and Abdul-Aziz (2015) utilized a combination of correlation analysis, principal component analysis (PCA), FA, and PLSR modeling to relatively link half-hourly $\mathrm{CO}_{2}$ fluxes with climatic and environmental variables for eight United States deciduous forests. Similar techniques were employed by Abdul-Aziz and Ahmed (2017) to compute the relative linkages of quarterly stream water quality with land use and hydrologic drivers for the coastal-urban watersheds in southeast Florida. Building on the methodology used in these studies, the current study applied a data analytics methodology to investigate the spatiotemporal similarity of climatic and ecohydrologic drivers of NEE. 


\section{CHAPTER 3: MATERIALS AND METHODS}

\subsection{Study sites}

Eddy covariance flux towers under the AmeriFlux network provide continuous observations of

NEE and the concurrent climatic and ecohydrological variables for a variety of ecosystem types.

The study was conducted for 57 AmeriFlux sites located in the United States (48 sites) and Canada

(9 sites) (Figure 3.1 and Table 3.1).

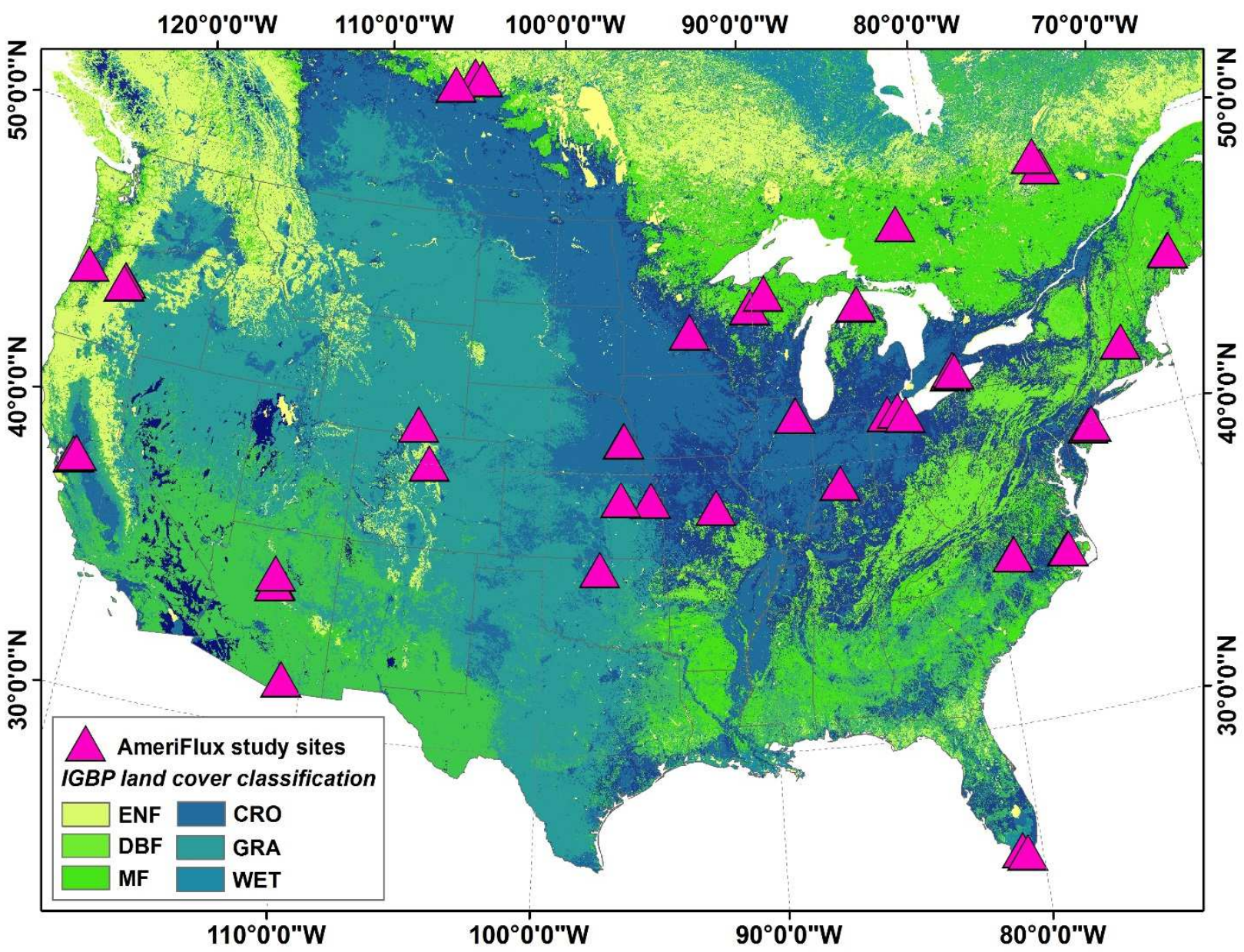

Figure 3.1: Locations of the 57 AmeriFlux study sites representing six diverse ecosystems. 
Table 3.1: Biophysical characteristics of the 57 AmeriFlux study sites representing six diverse ecosystems.

\begin{tabular}{|c|c|c|c|c|c|c|c|c|}
\hline Site name & $\begin{array}{l}\text { AmeriFlux } \\
\text { ID }\end{array}$ & $\begin{array}{l}\text { State/ } \\
\text { Province }\end{array}$ & Climate type & $\begin{array}{l}\text { Mean annual } \\
\text { precipitation } \\
(\mathrm{mm})\end{array}$ & $\begin{array}{l}\text { Mean annual } \\
\text { temperature } \\
\left({ }^{\circ} \mathrm{C}\right)\end{array}$ & $\begin{array}{l}\text { Sample } \\
\text { size }\end{array}$ & Period & Reference \\
\hline \multicolumn{9}{|l|}{ Evergreen Needleleaf Forest (ENF) } \\
\hline Duke Forest - loblolly pine & US-Dk3 & $\mathrm{NC}$ & $\begin{array}{l}\text { Humid } \\
\text { subtropical }\end{array}$ & 1170 & 14.36 & 13,529 & $2003-05$ & Stoy et al. 2009 \\
\hline Flagstaff - Managed Forest & US-Fmf & $\mathrm{AZ}$ & $\begin{array}{l}\text { Temperate } \\
\text { mediterranean }\end{array}$ & 546 & 9.50 & 18,278 & $2006-09$ & Dore et al. 2012 \\
\hline Flagstaff - Unmanaged Forest & US-Fuf & $\mathrm{AZ}$ & $\begin{array}{l}\text { Temperate } \\
\text { mediterranean }\end{array}$ & 562 & 8.70 & 16,891 & $2006-10$ & Kolb et al. 2013 \\
\hline GLEES & US-GLE & WY & $\begin{array}{l}\text { Subarctic } \\
\text { climate }\end{array}$ & 1200 & 0.80 & 10,698 & $2012-14$ & $\begin{array}{l}\text { Frank et al. } \\
2014\end{array}$ \\
\hline Howland Forest (main tower) & US-Ho1 & $\mathrm{ME}$ & $\begin{array}{l}\text { Temperate } \\
\text { continental }\end{array}$ & 1070 & 5.27 & 26,304 & $2002-13$ & $\begin{array}{l}\text { Davidson et al. } \\
2006\end{array}$ \\
\hline Howland Forest (west tower) & US-Ho2 & ME & $\begin{array}{l}\text { Temperate } \\
\text { mediterranean }\end{array}$ & 1064 & 5.13 & 24,977 & $2000-08$ & $\begin{array}{l}\text { Davidson et al. } \\
2006\end{array}$ \\
\hline Howland Forest (harvest site) & US-Ho3 & $\mathrm{ME}$ & $\begin{array}{l}\text { Temperate } \\
\text { mediterranean }\end{array}$ & 1072 & 5.31 & 8,375 & $2003-08$ & $\begin{array}{l}\text { Davidson et al. } \\
2006\end{array}$ \\
\hline $\begin{array}{l}\text { Metolius-intermediate aged } \\
\text { ponderosa pine }\end{array}$ & US-Me2 & OR & $\begin{array}{l}\text { Temperate } \\
\text { mediterranean }\end{array}$ & 523 & 6.28 & 43,662 & $2002-14$ & $\begin{array}{l}\text { Vickers et al. } \\
2012\end{array}$ \\
\hline Metolius-second young aged pine & US-Me3 & OR & $\begin{array}{l}\text { Temperate } \\
\text { mediterranean }\end{array}$ & 719 & 7.07 & 17,845 & $2004-08$ & $\begin{array}{l}\text { Vickers et al. } \\
2009\end{array}$ \\
\hline Metolius-first young aged pine & US-Me5 & OR & $\begin{array}{l}\text { Temperate } \\
\text { mediterranean }\end{array}$ & 591 & 6.47 & 12,473 & $2000-02$ & $\begin{array}{l}\text { Irvine et al. } \\
2004\end{array}$ \\
\hline Metolius Young Pine Burn & US-Me6 & OR & $\begin{array}{l}\text { Temperate } \\
\text { mediterranean }\end{array}$ & 494 & 7.59 & 7,348 & $2010-13$ & $\begin{array}{l}\text { Ruehr et al. } \\
2012\end{array}$ \\
\hline Mary's River (Fir) site & US-MRf & OR & $\begin{array}{l}\text { Temperate } \\
\text { mediterranean }\end{array}$ & 1820 & 10.24 & 13,878 & $2006-11$ & $*$ \\
\hline NC_Clearcut & US-NC1 & $\mathrm{NC}$ & $\begin{array}{l}\text { Humid } \\
\text { subtropical }\end{array}$ & 1320 & 16.6 & 15,388 & $2006-09$ & $\begin{array}{l}\text { Noormets et al. } \\
2012\end{array}$ \\
\hline NC_Loblolly Plantation & US-NC2 & $\mathrm{NC}$ & $\begin{array}{l}\text { Humid } \\
\text { subtropical }\end{array}$ & 1320 & 16.6 & 14,581 & $2006-08$ & $\begin{array}{l}\text { Noormets et al. } \\
2010\end{array}$ \\
\hline Niwot Ridge Forest & US-NR1 & $\mathrm{CO}$ & $\begin{array}{l}\text { subarctic } \\
\text { climate }\end{array}$ & 800 & 1.50 & 44,715 & $2006-14$ & $\begin{array}{l}\text { Desai et al. } \\
2011\end{array}$ \\
\hline Ontario - Turkey Point 2002 & CA-TP1 & $\mathrm{ON}$ & $\begin{array}{l}\text { Temperate } \\
\text { continental }\end{array}$ & 1036 & 8.00 & 27,410 & $2005-15$ & $\begin{array}{l}\text { Peichl et al. } \\
2010\end{array}$ \\
\hline
\end{tabular}


Table 3.1: continued

\begin{tabular}{|c|c|c|c|c|c|c|c|c|}
\hline Site name & $\begin{array}{l}\text { AmeriFlux } \\
\text { ID }\end{array}$ & $\begin{array}{l}\text { State/ } \\
\text { Province }\end{array}$ & Climate type & $\begin{array}{l}\text { Mean annual } \\
\text { precipitation } \\
(\mathrm{mm})\end{array}$ & $\begin{array}{l}\text { Mean annual } \\
\text { temperature } \\
\left({ }^{\circ} \mathrm{C}\right)\end{array}$ & $\begin{array}{l}\text { Sample } \\
\text { size }\end{array}$ & Period & Reference \\
\hline \multicolumn{9}{|l|}{ Evergreen Needleleaf Forest (ENF) } \\
\hline Ontario - Turkey Point 1989 & CA-TP2 & $\mathrm{ON}$ & $\begin{array}{l}\text { Temperate } \\
\text { continental }\end{array}$ & 1036 & 8.00 & 2,366 & $2002-03$ & $\begin{array}{l}\text { Peichl et al. } \\
2007\end{array}$ \\
\hline Ontario - Turkey Point 1939 & CA-TP4 & $\mathrm{ON}$ & $\begin{array}{l}\text { Temperate } \\
\text { continental }\end{array}$ & 1036 & 8.00 & 69,633 & $2002-15$ & $\begin{array}{l}\text { Arain and } \\
\text { Restrepo } 2005\end{array}$ \\
\hline $\begin{array}{l}\text { Quebec - Black Spruce/Jack Pine } \\
\text { Cutover }\end{array}$ & CA-Qcu & QC & $\begin{array}{l}\text { Subarctic } \\
\text { climate }\end{array}$ & 950 & 0.13 & 14,619 & $2005-10$ & $\begin{array}{l}\text { Coursolle et al. } \\
2012\end{array}$ \\
\hline Quebec - Mature Black Spruce & CA-Qfo & QC & $\begin{array}{l}\text { Subarctic } \\
\text { climate }\end{array}$ & 962 & -0.36 & 32,747 & $2004-10$ & $\begin{array}{l}\text { Bergeron et al. } \\
2007\end{array}$ \\
\hline Saskatchewan - Mature Black Spruce & CA-Obs & SK & $\begin{array}{l}\text { Subarctic } \\
\text { climate }\end{array}$ & 406 & 0.79 & 8,242 & $2001-10$ & $\begin{array}{l}\text { Amiro et al. } \\
2006\end{array}$ \\
\hline Saskatchewan - Mature Jack Pine & CA-Ojp & SK & $\begin{array}{l}\text { Subarctic } \\
\text { climate }\end{array}$ & 431 & 0.12 & 7,387 & $2004-10$ & $\begin{array}{l}\text { Amiro et al. } \\
2006\end{array}$ \\
\hline \multicolumn{9}{|l|}{ Deciduous Broadleaf Forest (DBF) } \\
\hline Duke Forest-hardwoods & US-Dk2 & $\mathrm{NC}$ & $\begin{array}{l}\text { Humid } \\
\text { subtropical }\end{array}$ & 1169 & 14.36 & 16,240 & $2003-05$ & $\begin{array}{l}\text { Novick et al. } \\
2009\end{array}$ \\
\hline Harvard Forest EMS Tower & US-Ha1 & MA & $\begin{array}{l}\text { Temperate } \\
\text { continental }\end{array}$ & 1071 & 6.62 & 23,366 & $2000-15$ & $\begin{array}{l}\text { Urbanski et al. } \\
2007\end{array}$ \\
\hline Morgan Monroe State Forest & US-MMS & IN & $\begin{array}{l}\text { Humid } \\
\text { subtropical }\end{array}$ & 1032 & 10.85 & 53,503 & $2001-14$ & $\begin{array}{l}\text { Dragoni et al. } \\
2011\end{array}$ \\
\hline Missouri Ozark Site & US-MOz & MO & $\begin{array}{l}\text { Humid } \\
\text { subtropical }\end{array}$ & 986 & 12.11 & 18,358 & $2010-14$ & Gu et al. 2006 \\
\hline Oak Openings & US-Oho & $\mathrm{OH}$ & $\begin{array}{l}\text { Warm } \\
\text { continental }\end{array}$ & 849 & 10.10 & 29,725 & $2006-13$ & Xie et al. 2014 \\
\hline Saskatchewan - Mature Aspen & CA-Oas & SK & $\begin{array}{l}\text { Subarctic } \\
\text { climate }\end{array}$ & 429 & 0.34 & 45,732 & $2000-10$ & $\begin{array}{l}\text { Amiro et al. } \\
2006\end{array}$ \\
\hline Silas Little & US-Slt & NJ & $\begin{array}{l}\text { Warm } \\
\text { continental }\end{array}$ & 1138 & 11.04 & 13,391 & $2011-14$ & $\begin{array}{l}\text { Clark et al. } \\
2014\end{array}$ \\
\hline $\begin{array}{l}\text { Univ. of Mich. Biological Station } \\
\text { (UMBS) }\end{array}$ & US-UMB & MI & $\begin{array}{l}\text { Temperate } \\
\text { continental }\end{array}$ & 803 & 5.83 & 42,232 & $2002-14$ & $\begin{array}{l}\text { Gough et al. } \\
2013\end{array}$ \\
\hline UMBS Disturbance & US-UMd & MI & $\begin{array}{l}\text { Temperate } \\
\text { continental }\end{array}$ & 803 & 5.83 & 14,768 & $2008-10$ & $\begin{array}{l}\text { Gough et al. } \\
2013\end{array}$ \\
\hline Willow Creek & US-WCr & WI & $\begin{array}{l}\text { Temperate } \\
\text { continental }\end{array}$ & 787 & 4.02 & 14,849 & $2001-06$ & Desai 2010 \\
\hline
\end{tabular}


Table 3.1: continued

\begin{tabular}{|c|c|c|c|c|c|c|c|c|}
\hline Site name & $\begin{array}{l}\text { AmeriFlux } \\
\text { ID }\end{array}$ & $\begin{array}{l}\text { State/ } \\
\text { Province }\end{array}$ & Climate type & $\begin{array}{l}\text { Mean annual } \\
\text { precipitation } \\
(\mathrm{mm})\end{array}$ & $\begin{array}{l}\text { Mean annual } \\
\text { temperature } \\
\left({ }^{\circ} \mathrm{C}\right)\end{array}$ & $\begin{array}{l}\text { Sample } \\
\text { size }\end{array}$ & Period & Reference \\
\hline \multicolumn{9}{|l|}{ Mixed Forest (MF) } \\
\hline Ontario - Boreal Mixedwood Forest & CA-Gro & $\mathrm{ON}$ & $\begin{array}{l}\text { Temperate } \\
\text { continental }\end{array}$ & 831 & 1.30 & 7,499 & $2006-12$ & $\begin{array}{l}\text { McCaughey et } \\
\text { al. } 2006\end{array}$ \\
\hline Sylvania Wilderness Area & US-Syv & MI & $\begin{array}{l}\text { Temperate } \\
\text { continental }\end{array}$ & 826 & 3.81 & 13,433 & $2003-06$ & Desai 2010 \\
\hline \multicolumn{9}{|l|}{ Cropland (CRO) } \\
\hline $\begin{array}{l}\text { ARM Southern Great Plains site- } \\
\text { Lamont }\end{array}$ & US-ARM & $\mathrm{OK}$ & $\begin{array}{l}\text { Humid } \\
\text { subtropical }\end{array}$ & 843 & 14.76 & 8,733 & $2010-13$ & $\begin{array}{l}\text { Raz-Yaseef et } \\
\text { al. } 2015\end{array}$ \\
\hline Curtice Walter-Berger cropland & US-CRT & $\mathrm{OH}$ & $\begin{array}{l}\text { Warm } \\
\text { continental }\end{array}$ & 849 & 10.10 & 12,860 & $2011-13$ & Chu et al. 2014 \\
\hline Fermi - Batavia (Agricultural site) & US-IB1 & IL & $\begin{array}{l}\text { Warm } \\
\text { continental }\end{array}$ & 929 & 9.18 & 28,067 & $2006-11$ & $\begin{array}{l}\text { Matamala et al. } \\
2008\end{array}$ \\
\hline Mead - irrigated continuous maize & US-Ne1 & $\mathrm{NE}$ & $\begin{array}{l}\text { Warm } \\
\text { continental }\end{array}$ & 790 & 10.07 & 44,881 & $2002-11$ & $\begin{array}{l}\text { Suyker et al. } \\
2005\end{array}$ \\
\hline $\begin{array}{l}\text { Mead - irrigated maize-soybean } \\
\text { rotation }\end{array}$ & US-Ne2 & $\mathrm{NE}$ & $\begin{array}{l}\text { Warm } \\
\text { continental }\end{array}$ & 789 & 10.08 & 37,565 & $2005-12$ & $\begin{array}{l}\text { Suyker et al. } \\
2005\end{array}$ \\
\hline $\begin{array}{l}\text { Mead - rainfed maize-soybean } \\
\text { rotation }\end{array}$ & US-Ne3 & $\mathrm{NE}$ & $\begin{array}{l}\text { Warm } \\
\text { continental }\end{array}$ & 784 & 10.11 & 38,068 & $2005-12$ & $\begin{array}{l}\text { Suyker et al. } \\
2005\end{array}$ \\
\hline Twitchell Corn & US-Tw2 & CA & $\begin{array}{l}\text { Warm } \\
\text { mediterranean }\end{array}$ & 421 & 15.50 & 4,286 & $2012-13$ & $\begin{array}{l}\text { Knox et al. } \\
2015\end{array}$ \\
\hline Twitchell Alfalfa & US-Tw3 & $\mathrm{CA}$ & $\begin{array}{l}\text { Warm } \\
\text { mediterranean }\end{array}$ & 421 & 15.60 & 13,052 & $2013-15$ & $*$ \\
\hline Twitchell Island & US-Twt & $\mathrm{CA}$ & $\begin{array}{l}\text { Warm } \\
\text { mediterranean }\end{array}$ & 421 & 15.60 & 26,217 & $2009-15$ & $\begin{array}{l}\text { Knox et al. } \\
2016\end{array}$ \\
\hline \multicolumn{9}{|l|}{ Grassland (GRA) } \\
\hline Duke Forest-open field & US-Dk1 & $\mathrm{NC}$ & $\begin{array}{l}\text { Humid } \\
\text { subtropical }\end{array}$ & 1170 & 14.36 & 18,148 & $2003-06$ & $\begin{array}{l}\text { Novick et al. } \\
2004\end{array}$ \\
\hline Flagstaff - Wildfire & US-Fwf & $\mathrm{AZ}$ & $\begin{array}{l}\text { Temperate } \\
\text { mediterranean }\end{array}$ & 557 & 8.40 & 24,971 & $2005-10$ & Dore et al. 2012 \\
\hline Fermi - Batavia (Prairie site) & US-IB2 & IL & $\begin{array}{l}\text { Warm } \\
\text { continental }\end{array}$ & 930 & 9.04 & 23,013 & $2007-11$ & $\begin{array}{l}\text { Matamala et al. } \\
2008\end{array}$ \\
\hline Kansas Field Station & US-KFS & KS & $\begin{array}{l}\text { Humid } \\
\text { subtropical }\end{array}$ & 1014 & 12.00 & 4,840 & $2009-10$ & $*$ \\
\hline
\end{tabular}


Table 3.1: continued

\begin{tabular}{|c|c|c|c|c|c|c|c|c|}
\hline Site name & $\begin{array}{l}\text { AmeriFlux } \\
\text { ID }\end{array}$ & $\begin{array}{l}\text { State/ } \\
\text { Province }\end{array}$ & Climate type & $\begin{array}{l}\text { Mean annual } \\
\text { precipitation } \\
(\mathrm{mm})\end{array}$ & $\begin{array}{l}\text { Mean annual } \\
\text { temperature } \\
\left({ }^{\circ} \mathrm{C}\right)\end{array}$ & $\begin{array}{l}\text { Sample } \\
\text { size }\end{array}$ & Period & Reference \\
\hline \multicolumn{9}{|l|}{ Grassland (GRA) } \\
\hline Konza Prairie & US-Kon & $\mathrm{KS}$ & $\begin{array}{l}\text { Humid } \\
\text { subtropical }\end{array}$ & 867 & 12.77 & 21,635 & $2006-12$ & $\begin{array}{l}\text { Danner et al. } \\
2003\end{array}$ \\
\hline KUOM Turfgrass Field & US-KUT & $\mathrm{MN}$ & $\begin{array}{l}\text { Warm } \\
\text { continental }\end{array}$ & 777 & 7.90 & 8,312 & $2007-09$ & $\begin{array}{l}\text { Hiller et al. } \\
2011\end{array}$ \\
\hline Sherman Island & US-Snd & $\mathrm{CA}$ & $\begin{array}{l}\text { Warm } \\
\text { mediterranean }\end{array}$ & 358 & 15.60 & 33,343 & $2007-14$ & $\begin{array}{l}\text { Hatala et al. } \\
2012\end{array}$ \\
\hline Santa Rita Grassland & US-SRG & $\mathrm{AZ}$ & Semi-arid & 420 & 17.00 & 47,178 & $2008-15$ & Scott et al. 2015 \\
\hline \multicolumn{9}{|l|}{ Wetland (WET) } \\
\hline Everglades (long hydroperiod marsh) & US-Elm & FL & Tropical & 1430 & 23.90 & 14,808 & $2008-13$ & $\begin{array}{l}\text { Schedlbauer et } \\
\text { al. } 2012\end{array}$ \\
\hline $\begin{array}{l}\text { Everglades (short hydroperiod } \\
\text { marsh) }\end{array}$ & US-Esm & FL & Tropical & 1430 & 23.90 & 16,417 & $2008-13$ & $\begin{array}{l}\text { Schedlbauer et } \\
\text { al. } 2012\end{array}$ \\
\hline Mayberry Wetland & US-Myb & $\mathrm{CA}$ & $\begin{array}{l}\text { Warm } \\
\text { mediterranean }\end{array}$ & 338 & 15.90 & 18,442 & $2013-15$ & $\begin{array}{l}\text { Matthes et al. } \\
2015\end{array}$ \\
\hline Twitchell Wetland West Pond & US-Tw1 & $\mathrm{CA}$ & $\begin{array}{l}\text { Warm } \\
\text { mediterranean }\end{array}$ & 421 & 15.50 & 12,949 & $2012-15$ & $\begin{array}{l}\text { Miller et al. } \\
2010\end{array}$ \\
\hline Twitchell East End Wetland & US-Tw4 & CA & $\begin{array}{l}\text { Warm } \\
\text { mediterranean }\end{array}$ & 421 & 15.60 & 8,644 & $2014-15$ & $*$ \\
\hline Winous Point North Marsh & US-WPT & $\mathrm{OH}$ & $\begin{array}{l}\text { Warm } \\
\text { continental }\end{array}$ & 849 & 10.10 & 14,319 & $2011-13$ & Chu et al. 2015 \\
\hline
\end{tabular}


The study sites represented six diverse ecosystems; evergreen needleleaf forest (ENF), deciduous broadleaf forest (DBF), mixed forest (MF), cropland (CRO), grassland (GRA), and wetland (WET). The ecosystems are classified according to the International Geosphere-Biosphere Programme (IGBP) for land cover classification. ENF, DBF, and MF are defined as the terrestrial land surfaces dominated by $>60 \%$ of woody vegetation having plant heights exceeding 2 meters. ENF exhibits green foliage all year round whereas the plants in DBF are characterized by the annual cycle of leaf-on and leaf-off periods. MF combines the characteristics of both ENF and DBF. CRO represents land covered with crops that go through harvesting at certain periods of the year. GRA is herbaceous land cover that comprises $<10 \%$ of trees and shrubs. WET comprises of a combination of water, herbaceous, and woody vegetation (Kim, 2013).

The Florida Everglades short hydro-period marsh (US-ESM) was selected for investigating the multi-temporal linkages of NEE as a case study site. The site is located within the Taylor slough region of the Everglades national park (ENP) (Figure 3.2) and is classified as a permanent wetland having a yearlong growing season (AmeriFlux, 2016, Schedlbauer et al., 2011). The study site is an oligotrophic freshwater marsh ecosystem that is seasonally flooded approximately 5 months in each year (Obeysekera et al., 1999, Schedlbauer et al., 2012). The seasonal flooding of this site along with the year round productivity contribute to its highly dynamic characteristics. 


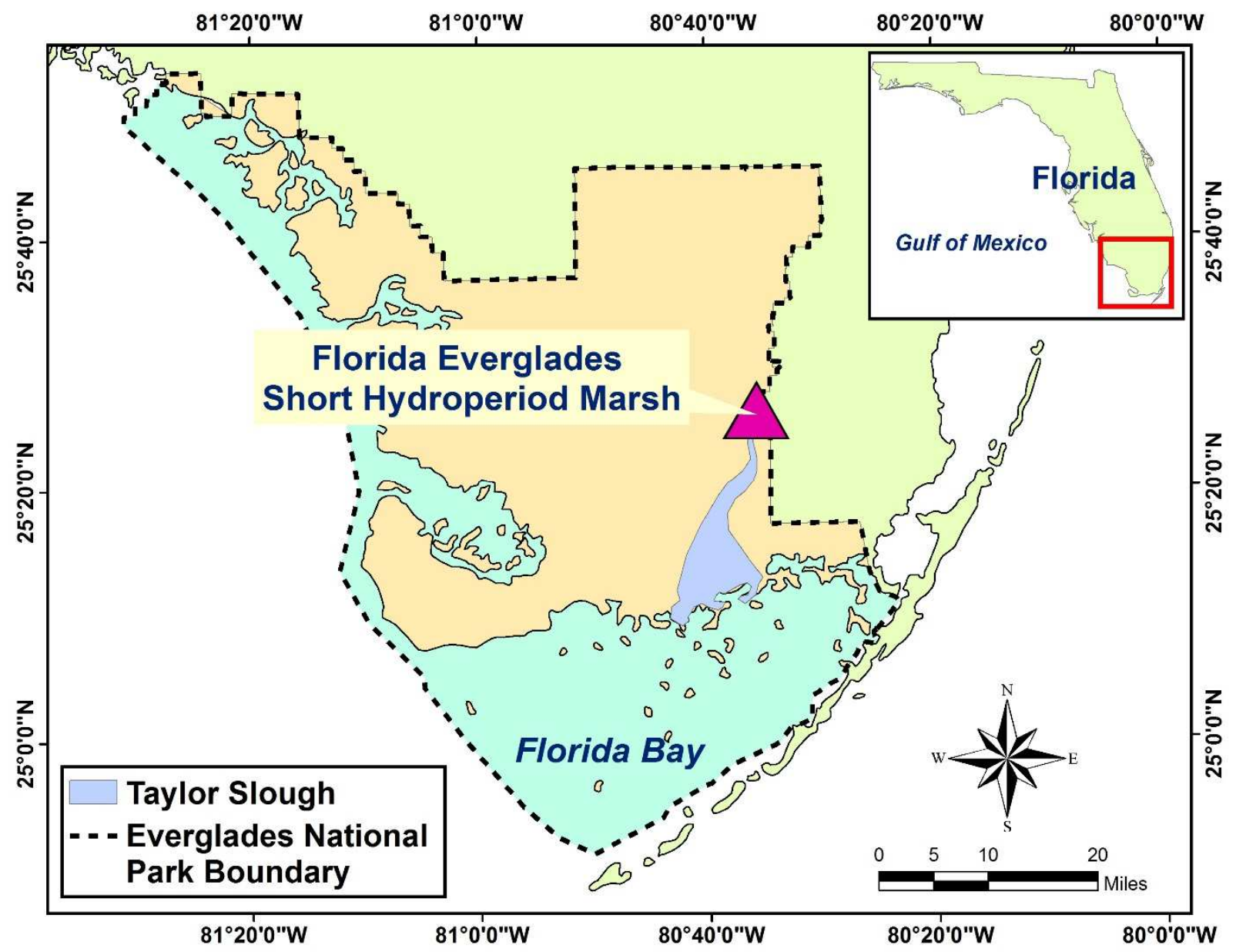

Figure 3.2: Location of the case study site at Florida everglades short hydro-period marsh.

\subsection{Data sets}

Fine resolution (hourly or half-hourly average) observations of NEE and the corresponding climatic and ecohydrological variables were obtained from the AmeriFlux network (AmeriFlux, 2016) for the period of 2000-2015. Among the 57 sites, six of the sites (Harvard forest, Morgan Monroe forest, University Michigan biological station, Mead - irrigated continuous maize, Mead - irrigated maize-soybean rotation, and Mead - rainfed maize-soybean rotation) reported hourly data whereas the remaining sites reported half-hourly data. For the final data set to maintain temporal uniformity across all the sites, hourly time-series data were determined for the remaining 
51 sites by averaging every two half-hourly observations. The final data set for each site therefore consisted hourly observations of NEE along with the 12 climatic and ecohydrological variables: net radiation (Rn), photosynthetically active radiation (PAR), latent heat flux (LE), sensible heat flux $(H)$, ground heat flux $(\mathrm{G})$, air temperature (TA), soil temperature (TS), vapor pressure deficit (VPD), soil water content (SWC), wind speed (WS), friction velocity (USTAR), and ambient atmospheric $\mathrm{CO}_{2}$ concentration $\left(\mathrm{CO}_{2}\right)$. SWC was selected here as a substitute of precipitation as it lacks consistent variation at the hourly temporal scale. Signs of the flux variables followed AmeriFlux conventions; where NEE, LE, and H denoted upward flux (leaving the system/release) when positive and downward flux (entering the system/uptake) when negative. On the contrary, positive values of Rn, PAR, and G indicated downward fluxes when negative and vice versa. Considering the importance of water level (WL) in the Everglades short hydro-period site as discussed in chapter 2, it was included in the multi-temporal linkage analysis. Hourly time series observational WL data was collected from the Everglades depth estimation network (EDEN) database (EDEN, 2016) and merged with the other 12 climatic and ecohyrdological variables of the Everglades short hydro-period marsh site to form a complete data set for the multi-temporal linkage analysis.

In the study, Level 2 AmeriFlux data with gaps was used. The data passed through quality control and assurance checks. Data with gaps were used for this study to avoid the potential synthetic biases associated with gap-filling. The following two-layered data-filtering approach was implemented to remove erroneous data (i.e., systematic errors occurring at nighttime stable conditions) and extreme outliers: 
Layer-1: Hourly NEE observations were filtered for nighttime low turbulence conditions. Steps recommended by Aubinet et al. (2012) were followed to determine the nighttime USTAR threshold separately for each year of each site. This layer of filtering involved the following steps.

Step-1: Available nighttime hourly NEE observations were sorted by the ascending order of USTAR.

Step-2: Correlation between USTAR and the major respiration-driving variable (TA) was computed and checked against a threshold correlation coefficient of 0.40 to identify any notable covariation of the two. For all the years at each site of this study, the computed correlations were below the threshold value; so additional treatment (normalization by temperature) of USTAR data was not necessary.

Step-3: The sorted USTAR data were grouped into 30 classes and then the mean NEE was calculated for each class.

Step-4: Mean NEE in each USTAR class was compared with the average of mean NEE at all higher classes. A particular class of USTAR was identified as the threshold class when the corresponding mean NEE was substantially different from the average of mean NEE for all the higher classes. The difference between mean NEE for a particular class and the mean NEE of all higher classes was considered substantial when it represented the 75 percentile of total 29 differences representing the 30 classes of USTAR.

Step-5: The median of USTAR values from the identified threshold class was taken as the USTAR threshold. If the computed USTAR threshold was below $0.1 \mathrm{~m} / \mathrm{s}$, it was used as the minimum threshold to filter nighttime NEE. 
Layer-2: $1^{\text {st }}$ and $99^{\text {th }}$ percentile values were used as identifiers of extreme outliers. Therefore, extreme outliers for each variable were removed below their $1^{\text {st }}$ percentile and above their $99^{\text {th }}$ percentile thresholds for each year at a particular site.

After the two-layered data filtering procedure was applied, if the data matrix of a site consisted of gaps along the 1-hour, 1-day, 8-day, 15-day, and 30-day observational panel that entire panel was removed to maintain a complete data matrix. Among the 57 sites, $23 \%$ of the sites were missing G, 14\% missing SWC, and $11 \%$ of the sites were missing both G and SWC. The sample size of the data sets of the 57 sites varied between 2,366 and 69,633. Mean NEE of the sites under each ecosystem represented $\mathrm{CO}_{2}$ uptake signal at the 1-hour scale (Table 3.2). 
Table 3.2: Data summary of observed hourly NEE, climatic, and ecohydrological variables by ecosystem type.

\begin{tabular}{|c|c|c|c|c|c|c|c|c|c|c|c|c|}
\hline \multirow{2}{*}{$\begin{array}{l}\text { Ecosystems } \\
\text { Variables }\end{array}$} & \multicolumn{2}{|c|}{ ENF } & \multicolumn{2}{|c|}{$\mathrm{DBF}$} & \multicolumn{2}{|c|}{ MF } & \multicolumn{2}{|c|}{$\mathrm{CRO}$} & \multicolumn{2}{|c|}{ GRA } & \multicolumn{2}{|c|}{ WET } \\
\hline & Mean & $\begin{array}{l}\text { Standard } \\
\text { deviation }\end{array}$ & Mean & $\begin{array}{l}\text { Standard } \\
\text { deviation }\end{array}$ & Mean & $\begin{array}{l}\text { Standard } \\
\text { deviation }\end{array}$ & Mean & $\begin{array}{l}\text { Standard } \\
\text { deviation }\end{array}$ & Mean & $\begin{array}{l}\text { Standard } \\
\text { deviation }\end{array}$ & Mean & $\begin{array}{l}\text { Standard } \\
\text { deviation }\end{array}$ \\
\hline $\mathrm{NEE}\left(\mu \mathrm{mol} / \mathrm{m}^{2} / \mathrm{s}\right)$ & -1.36 & -5.36 & -1.83 & -6.89 & -0.28 & -4.2 & -1.13 & -8.46 & -0.32 & -4.53 & -0.72 & -4.58 \\
\hline $\mathrm{Rn}\left(\mathrm{W} / \mathrm{m}^{2}\right)$ & 109.97 & -204.26 & 110.6 & -191.15 & 63.37 & -157.6 & 112.31 & -194.32 & 95.38 & -197.08 & 159.17 & -252.95 \\
\hline $\operatorname{PAR}\left(\mu \mathrm{mol} / \mathrm{m}^{2} / \mathrm{s}\right)$ & 411.31 & -526.64 & 393.66 & -491.45 & 287.02 & -440.45 & 440.79 & -535.06 & 453.76 & -566.42 & 514.38 & -602.46 \\
\hline $\mathrm{LE}\left(\mathrm{W} / \mathrm{m}^{2}\right)$ & 41.49 & -59.89 & 44.55 & -76.17 & 24.52 & -47.39 & 64.61 & -89.66 & 48.28 & -68.18 & 90.46 & -87.47 \\
\hline $\mathrm{H}\left(\mathrm{W} / \mathrm{m}^{2}\right)$ & 45.02 & -110.01 & 37.55 & -87.8 & 21.54 & -73.11 & 23.37 & -71.94 & 30.77 & -85.04 & 25 & -68.88 \\
\hline $\mathrm{G}\left(\mathrm{W} / \mathrm{m}^{2}\right)$ & 0.76 & -11.19 & 0.86 & -8.15 & -0.83 & -13.73 & 5.24 & -38.94 & 2.22 & -37.71 & 2.63 & -18.25 \\
\hline TA $\left({ }^{\circ} \mathrm{C}\right)$ & 8.07 & -10.58 & 8.79 & -11.42 & 3.93 & -11.77 & 12.6 & -10.98 & 15.03 & -9.57 & 19.67 & -8.14 \\
\hline $\operatorname{TS}\left({ }^{\circ} \mathrm{C}\right)$ & 8.67 & -8.12 & 9.34 & -7.33 & 6.6 & -6.77 & 12.91 & -9.53 & 15.12 & -8.54 & 19.34 & -6.55 \\
\hline VPD $(\mathrm{KPa})$ & 0.51 & -0.55 & 0.47 & -0.5 & 0.3 & -0.39 & 0.65 & -0.67 & 0.96 & -0.84 & 0.86 & -0.61 \\
\hline SWC (\%) & 17.16 & -10.78 & 24.43 & -13.62 & 27.59 & -11.39 & 30.96 & -14.36 & 26.83 & -15.65 & 78.73 & -15.3 \\
\hline $\mathrm{WS}(\mathrm{m} / \mathrm{s})$ & 2.87 & -1.96 & 3.16 & -1.36 & 3.3 & -1.14 & 3.87 & -2.04 & 3.18 & -1.85 & 2.99 & -1.5 \\
\hline $\operatorname{USTAR}(\mathrm{m} / \mathrm{s})$ & 0.51 & -0.35 & 0.55 & -0.25 & 0.52 & -0.26 & 0.31 & -0.16 & 0.32 & -0.17 & 0.35 & -0.18 \\
\hline $\mathrm{CO}_{2}(\mu \mathrm{mol} / \mathrm{mol})$ & 383.88 & -17.64 & 388.79 & -16.47 & 386.35 & -10.79 & 388.11 & -19.87 & 384.79 & -16.61 & 393.5 & -15.5 \\
\hline
\end{tabular}

Notes: ENF, DBF, MF, CRO, GRA, and WET, refer to evergreen needleleaf forests, deciduous broadleaf forests, mixed forests, grasslands, croplands, and wetlands, respectively. 
The hourly filtered data set of the Everglades short hydro-period marsh site was averaged over the hours to obtain the data at the four different temporal scales (1-day, 8-day, 15-day, and 30-day). Mean NEE in all the temporal scales represented $\mathrm{CO}_{2}$ uptake for the Florida Everglades short hydro-period site (Table 3.3).

Table 3.3: Data summary of NEE, climatic, and ecohydrological variables at the different temporal scales for the Florida Everglades short hydro-period marsh site.

\begin{tabular}{|c|c|c|c|c|c|c|c|c|}
\hline \multirow{2}{*}{$\begin{array}{l}\text { Time scale } \\
\text { Variables }\end{array}$} & \multicolumn{2}{|c|}{$\begin{array}{c}\text { 1-day } \\
\mathrm{N}=1443\end{array}$} & \multicolumn{2}{|c|}{$\begin{array}{c}\text { 8-day } \\
\mathrm{N}=202\end{array}$} & \multicolumn{2}{|c|}{$\begin{array}{l}\text { 15-day } \\
\mathrm{N}=113\end{array}$} & \multicolumn{2}{|c|}{$\begin{array}{l}\text { 30-day } \\
\mathrm{N}=59\end{array}$} \\
\hline & Mean & $\begin{array}{l}\text { Standard } \\
\text { deviation }\end{array}$ & Mean & $\begin{array}{l}\text { Standard } \\
\text { deviation }\end{array}$ & Mean & $\begin{array}{l}\text { Standard } \\
\text { deviation }\end{array}$ & Mean & $\begin{array}{l}\text { Standard } \\
\text { deviation }\end{array}$ \\
\hline $\mathrm{NEE}\left(\mu \mathrm{mol} / \mathrm{m}^{2} / \mathrm{s}\right)$ & -1.08 & 1.05 & -1.04 & 0.86 & -1.01 & 0.79 & -0.97 & 0.76 \\
\hline $\mathrm{Rn}\left(\mathrm{W} / \mathrm{m}^{2}\right)$ & 210.42 & 84.79 & 200.10 & 59.08 & 197.11 & 53.31 & 192.51 & 50.36 \\
\hline $\operatorname{PAR}\left(\mu \mathrm{mol} / \mathrm{m}^{2} / \mathrm{s}\right)$ & 599.30 & 211.58 & 573.02 & 143.61 & 566.65 & 127.49 & 554.37 & 119.77 \\
\hline $\mathrm{LE}\left(\mathrm{W} / \mathrm{m}^{2}\right)$ & 98.20 & 39.54 & 97.96 & 30.53 & 97.14 & 29.37 & 94.30 & 27.45 \\
\hline $\mathrm{H}\left(\mathrm{W} / \mathrm{m}^{2}\right)$ & 72.15 & 53.17 & 72.19 & 43.54 & 73.20 & 42.37 & 71.22 & 39.99 \\
\hline $\mathrm{G}\left(\mathrm{W} / \mathrm{m}^{2}\right)$ & 5.83 & 12.83 & 5.11 & 10.04 & 4.62 & 9.22 & 3.97 & 8.72 \\
\hline $\mathrm{TA}\left({ }^{\circ} \mathrm{C}\right)$ & 25.12 & 4.55 & 24.95 & 4.09 & 24.72 & 3.93 & 24.40 & 3.81 \\
\hline $\mathrm{TS}\left({ }^{\circ} \mathrm{C}\right)$ & 24.02 & 4.38 & 24.01 & 4.28 & 23.84 & 4.20 & 23.62 & 4.16 \\
\hline VPD (KPa) & 0.94 & 0.29 & 0.92 & 0.20 & 0.91 & 0.17 & 0.90 & 0.15 \\
\hline $\mathrm{WL}(\mathrm{ft})$ & 2.84 & 1.31 & 2.84 & 1.33 & 2.79 & 1.34 & 2.79 & 1.32 \\
\hline SWC (\%) & 68.15 & 15.71 & 67.85 & 15.86 & 67.14 & 16.10 & 67.07 & 15.69 \\
\hline $\mathrm{WS}(\mathrm{m} / \mathrm{s})$ & 2.78 & 0.82 & 2.79 & 0.57 & 2.80 & 0.50 & 2.80 & 0.43 \\
\hline USTAR (m/s) & 0.31 & 0.08 & 0.31 & 0.05 & 0.32 & 0.05 & 0.32 & 0.04 \\
\hline $\mathrm{CO}_{2}(\mu \mathrm{mol} / \mathrm{mol})$ & 392.87 & 11.79 & 392.72 & 8.68 & 392.54 & 7.70 & 392.28 & 6.60 \\
\hline
\end{tabular}

Note: 'N' represents the sample size at each of the temporal scales. 


\subsection{Data analytics}

The framework of data analytics implemented in this study, involved a systematic and sequential application of three methods; Pearson's correlation analysis, factor analysis (FA) (Jolliffe, 2002, Mahbub et al., 2010), and partial least squares regression (PLSR) modeling (Wold et al., 2001). The correlation analysis determined the linear relationships of the participatory variables along with the multicollinear structure of the data matrix at each site. FA on the other hand, provided multivariate interrelations and grouping patterns among the predictor and response variables unaffected by multicollinearity. Finally, the application of optimal PLSR modeling resulted in the appropriate estimations of climatic and ecohyrologic linkages of NEE. The data-analytics methodology is summarized in the following flow diagram (Figure 3.3).

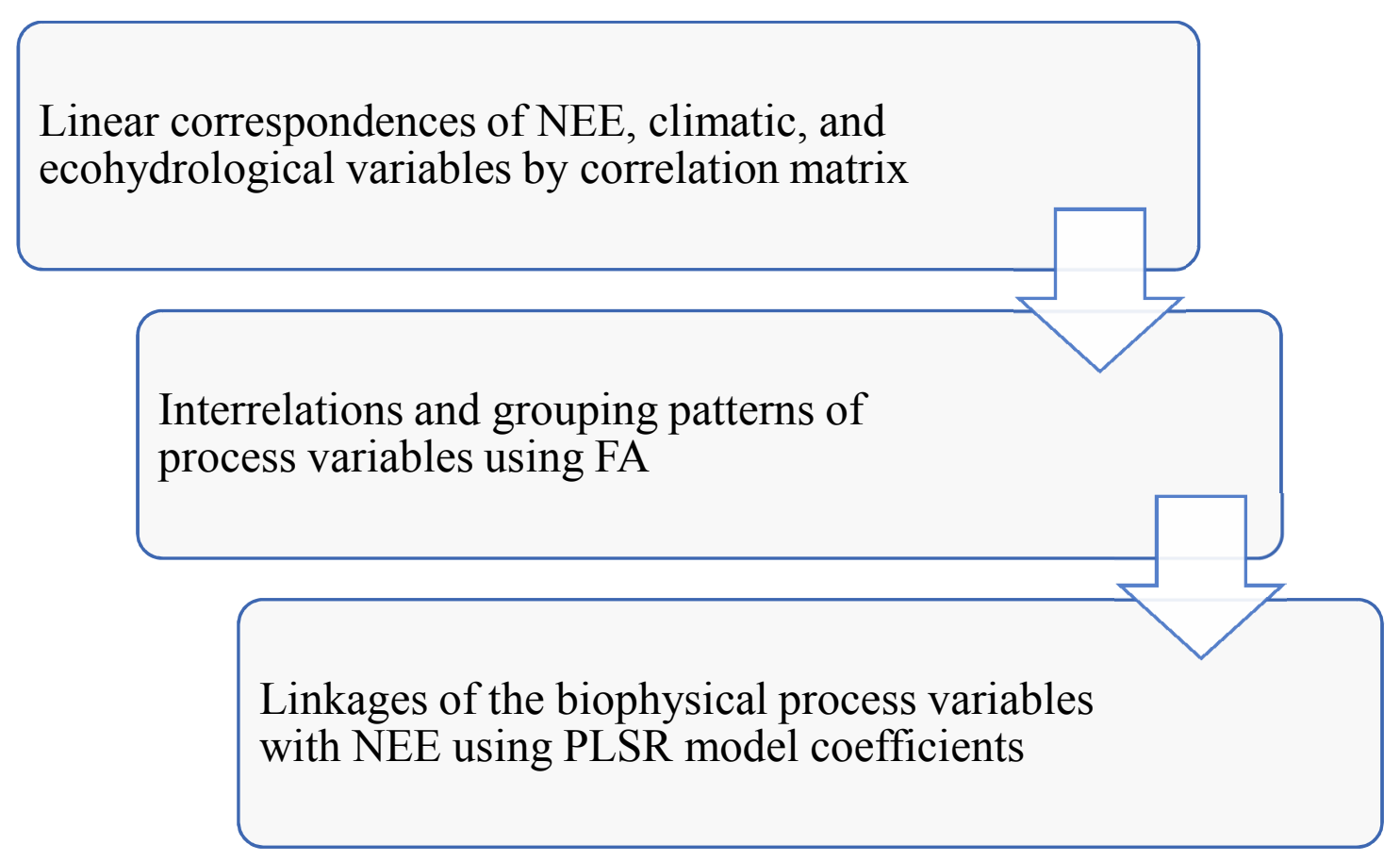

Figure 3.3: Stepwise representation of the data analytics approach to determine the climatic and ecohyrologic linkages of NEE. 
Before applying the data analytics approach, data matrix of each site, which was Ztransformed $\left(Z=(X-\bar{X}) / S_{X}\right.$, where $Z=$ standard normal variate, $X=$ original variable, $\bar{X}=$ site-specific mean of $X$ over the study period, and $S_{X}=$ standard deviation of X) to bring the different units of the different variables into a standardized domain. All the steps of the analysis was performed in MATLAB (R2016a).

\subsubsection{Pearson's correlation analysis}

Pearson's correlation determines the strength of the linear relationship between two quantitative variables. The correlation coefficients of NEE, climatic, and ecohydrological variables were determined to gather background information about the bivariate linear relationships among the participatory variables across different ecosystems. Pearson's correlation coefficient of a predictor variable (i.e., climatic/ecohydrological variable) did not represent its true relationship with the response variable (i.e., NEE) as that predictor was subjected to multicollinearity with other predictors. Multicollinearity would result in unreliable estimates of model parameters. Therefore, triangular correlation matrices of the participatory variables across the diverse ecosystems were determined to develop an initial understanding of their multicollinear structure.

\subsubsection{Factor analysis (FA)}

FA has been applied successfully in various data mining and analysis studies (Panda et al. 2006, Dragon, 2006). In this study, FA was employed to identify multivariate interrelations and grouping patterns of the participatory variables across diverse ecosystems. It decomposed the actual set of variables (i.e., NEE, climatic, and ecohydrological variables) into a smaller set of hidden quantities in orthogonal planes known as factors. This method resolved multicollinearity through orthogonal transformations by means of "varimax" rotation and delivered optimized loadings of the variables in each of the factors (Jolliffe, 2002). Therefore, each of the actual variables were indeed linear 
function of the hidden factors. If $p$ denoted the number of actual variables $\left(X_{1}, X_{2}, \ldots, X_{p}\right)$ and $m$ denoted the number of the hidden factors $\left(F_{1}, F_{2}, \ldots, F_{m}\right)$ then,

$$
X_{j}=a_{j 1} F_{1}+a_{j 2} F_{2}+\ldots \ldots+a_{j m} F_{m}
$$

Where, $j=1,2, \ldots, p$ and $a_{j 1}, a_{j 2}, \ldots, a_{j m}$ denoted the factor loadings. Here, $m$ should be less than or equal to $p$ (Jolliffe, 2002).

An eigenvalue criteria (eigenvalue $\geq 1$ ) was used to extract the least number of factors representing the most system variance of the data matrix combining the predictor and response variables (Ishtiaq and Abdul-Aziz, 2015). The knowledge of multivariate interrelations and grouping patterns obtained from FA were then synthesized to form biophysical process components of NEE.

\subsubsection{Partial least squares regression (PLSR) modeling}

PLSR is a sophisticated empirical modeling approach that can explain the linear relationships between response (i.e., y) and predictor (i.e., $\mathrm{x}$ ) variables by eliminating the instability of the model parameter ( $\beta$ coefficients) approximations caused by multicollinearity among the predictor variables. Using PLSR modeling NEE was estimated with the optimal number of partial least squares (PLS) components. Optimization of the PLSR models was necessary to reliably estimate $\beta$ coefficients by resolving multicollinearity among the predictor variables (Kuhn and Johnson, 2013).

There might be some debate as to why PLSR was preferred for the analysis of this study instead of applying the conventional principal component regression (PCR) as both the methods could handle multicollinearity in a data matrix. There are some fundamental differences between PCR and PLSR that made PLSR distinct for its application in the data analytics approach for large 
data sets. In PCR, the most relevant information present in the predictor variables (i.e., climatic and ecohydrological variables) are extracted by performing principal component analysis on the predictors and no information regarding the response variable (i.e., NEE) is taken into account. Therefore, PCR only pursues the variability that exists among the predictors. If that variability is associated with the variability of the response variable, then PCR could establish a successful prediction. However, if the variability identified in the predictor space could not recognize the variability of the response variable, then PCR would have difficulty to identify satisfactory prediction. In contrast, the components in PLSR is calculated by maximally establishing covariance between both the predictor and response variables. Therefore, in PLSR the information present in the response variable is also used in the initial stage of the algorithm with the predictors. Due to such inherent advantage, PLSR was chosen over PCR for the data analytics.

To quantify the climatic and ecohydrologic linkages of NEE, dimensionless PLSR models were developed using the Z-transformed data. The standardization of the data led to models with zero intercepts. The SIMPLS algorithm coupled with a 10-fold cross-validation was implemented to obtain robust estimations of the optimized PLSR model parameters (De Jong, 1993, Kuhn and Johnson, 2013). The SIMPLS algorithm is the simple modification of the PLS algorithm NIPALS (nonlinear iterative partial least squares) improved by De Jong (1993). In general, the perspective of the NIPALS algorithm shifted towards the deflation of the covariance matrix between the predictor and response variables instead of deflating the predictor matrix and response vector. A three-fold criterion based synthesis was executed to determine the minimum number of PLS components to attain optimal PLSR models. The three-fold criterion consisted of maximum NashSutcliffe efficiency (NSE), minimum Akaike Information Criterion (AIC) (Akaike, 1974, AbdulAziz and Ishtiaq, 2014), and the eigenvalue $\geq 1$ criteria. Due to the different sample sizes at 
different sites, normalized AIC was determined to maintain equilibrium at all sites. Therefore, AIC was defined as,

$$
A I C_{N E E}(p)=\ln \left(\frac{S S E_{N E E}}{N}\right)+\frac{2 p}{N}
$$

Here, $p$ was the number of PLS components, $N$ was the number of observations (sample size), and $S S E_{N E E}$ was the total sum of squared error upon estimation of NEE.

An important part of empirical modeling in this study was determining the uncertainty of $\beta$. The uncertainty of $\beta$ estimations also gave idea about the global sensitivities at the same time. Monte-Carlo based uncertainty analysis has become a universal tool for assessing such sensitivity of models (Janssen, 2013). For the uncertainty analysis, optimal PLSR models at each site (for multi-temporal analysis, at each temporal scale) were reiterated 1000 times through bootstrap resampling. Therefore, the total number of observations at a particular site after bootstrapping was equal to 1000 times the original sample size of that site. The bootstrap Monte-Carlo process resulted in 1000 approximations of optimized PLSR model $\beta$. The mean of the estimated 1000 set of $\beta$ for each variable represented their linkages with NEE. Their sign (i.e., positive or negative) revealed the nature of their influence on $\mathrm{CO}_{2}$ exchange between the biosphere and the atmosphere (i.e., positive for influence on $\mathrm{CO}_{2}$ release and negative for influence on $\mathrm{CO}_{2}$ uptake). Standard deviations of each of the estimated 1000 set of $\beta$ exhibited the parameter uncertainty for each variable. The fitting efficiencies of the developed models were measured using NSE (NashSutcliffe efficiency) and the accuracy of model predictions by ratio of root-mean-square error to the standard deviation of observations (RSR): 


$$
\begin{gathered}
N S E=1-\frac{\sum_{i=1}^{N}\left(N E E_{i, o b s}-N E E_{i, \mathrm{mod}}\right)^{2}}{\sum_{i=1}^{N}\left(N E E_{i, o b s}-\overline{N E E_{o b s}}\right)^{2}} \\
R S R=\sqrt{\sum_{i=1}^{N}\left(N E E_{i, o b s}-N E E_{i, \text { mod }}\right)^{2}} / \sigma_{N E E, o b s}
\end{gathered}
$$

Where, $N$ is the number of observations, $N E E_{i, o b s}$ and $N E E_{i, \bmod }$ are the $i^{\text {th }}$ observed and predicted (respectively) Z-scores of NEE, and $N E E_{i, o b s}=0$ and, $\sigma_{N E E, o b s}=1.0$ are the average and standard deviation (respectively) of the observed Z-scores of NEE. The performance of the PLSR models were assessed by comparing the observed and modeled NEE. The RSR criteria described by Moriasi et al. (2007) was used here to evaluate the accuracies of the estimated models. According to the aforementioned paper, a perfect to very good model has an RSR between 0 and 0.50, a good model has an RSR between 0.50 and 0.60 , and a satisfactory model has an RSR between 0.60 and $0.70 ;$ RSR $>0.70$ indicated an unsatisfactory model.

Coupled with the information gathered from data analytics and process-based understandings of biological systems, the PLSR model $\beta$ of the NEE drivers were aggregated to attain the linkages of different biophysical process components. Each of the aggregated linkages represented the strength of that component to drive NEE. The 95\% upper and lower confidence limits of the aggregated linkages at each site were determined to express the uncertainty of the biophysical process component linkages. 


\section{CHAPTER 4: RESULTS OF HOURLY LINKAGES OF NEE ACROSS DIVERSE ECOSYSTEMS}

\subsection{Linear relationships from correlation analysis}

Pearson's correlation matrices for the 57 sites were computed to explore the bivariate linear relationships of the participatory variables across the diverse ecosystems at the 1-hour temporal scale. The correlation matrix at each site also provided information on the multicollinear structure of their respective data matrix. The correlation coefficients of the variables were averaged under their respective ecosystems (Table 4.1). The radiation variables exhibited moderate to high mean correlations ( $\mathrm{Rn} ; r=-0.57$ to -0.73 and PAR; $r=-0.55$ to -0.72 ) with NEE. The energy and heat flux variables showed both weak and strong correlations across the diverse ecosystems, where LE was correlated highly ( $r=-0.63$ to -0.79$), \mathrm{H}$ moderately to highly ( $r=-0.23$ to -0.62 ), and $\mathrm{G}$ weakly to moderately ( $r=-0.02$ to -0.58$)$ with NEE. The temperature variables along with VPD showed weak to moderate correlations (TA; $r=-0.23$ to -0.42 , TS; $r=-0.11$ to -0.41 , and VPD; $r$ $=-0.29$ to -0.43 ) with NEE.

Table 4.1: Mean Pearson's correlation coefficients $(r)$ between NEE and the climatic and ecohydrological variables across diverse ecosystems.

\begin{tabular}{lllllllllllll}
\hline Ecosystems & Rn & PAR & LE & $\mathrm{H}$ & $\mathrm{G}$ & TA & TS & VPD & SWC & WS & USTAR & $\mathrm{CO}_{2}$ \\
\hline ENF & -0.73 & -0.72 & -0.72 & -0.62 & -0.46 & -0.28 & -0.19 & -0.36 & 0.01 & -0.09 & -0.22 & 0.24 \\
DBF & -0.66 & -0.65 & -0.79 & -0.37 & -0.30 & -0.42 & -0.41 & -0.40 & 0.20 & 0.13 & -0.04 & 0.40 \\
MF & -0.69 & -0.68 & -0.76 & -0.51 & -0.58 & -0.23 & -0.22 & -0.35 & 0.09 & 0.03 & -0.16 & 0.42 \\
CRO & -0.57 & -0.55 & -0.72 & -0.23 & -0.34 & -0.28 & -0.21 & -0.29 & 0.03 & 0.06 & -0.14 & 0.33 \\
GRA & -0.68 & -0.66 & -0.72 & -0.51 & -0.48 & -0.24 & -0.12 & -0.35 & 0.06 & -0.06 & -0.15 & 0.29 \\
WET & -0.68 & -0.69 & -0.63 & -0.58 & -0.02 & -0.34 & -0.11 & -0.43 & -0.01 & -0.11 & -0.22 & 0.24 \\
\hline
\end{tabular}

Notes: ENF, DBF, MF, CRO, GRA, and WET refer to evergreen needleleaf forests, deciduous broadleaf forests, mixed forests, grasslands, croplands, and wetlands, respectively. 
Ambient atmospheric $\mathrm{CO}_{2}$ also showed weak to moderate $(r=0.24$ to 0.42$)$ correlations with NEE. Comparatively weak correlations were observed for the aerodynamic variables (WS; $r$ $=-0.11$ to 0.13 and USTAR; $r=-0.04$ to -0.22$)$ and SWC $(r=-0.01$ to 0.20$)$.

Triangular correlation matrices comprised of mean correlation coefficients of the participatory variables under each ecosystem provided information on the multicollinearity among those variables (Figure 4.1). The radiation variables ( $\mathrm{Rn}$ and PAR) exhibited very strong ( $r=0.97$ to 0.99 ) interrelations among themselves whereas energy and heat flux variables (LE, H, and G) showed weak to strong interrelations ( $r=0.15$ to 0.68$)$ across the diverse ecosystems. The temperature variables (TA and TS) were interrelated very strongly ( $r=0.71$ to 0.91$)$. Comparatively moderate to strong interrelations ( $r=0.33$ to 0.78 ) were observed between the temperature variables and VPD. The aerodynamic variables (WS and USTAR) were also highly interrelated ( $r=0.77$ to 0.87$)$ among themselves. The interrelations between the radiation variables with the energy and heat flux variables (LE and $\mathrm{H})$ were also high $(r=0.73$ to 0.91$)$. Notable interrelations were observed between Rn, PAR, LE, and VPD that varied between moderate to strong ( $r=0.46$ to 0.72$)$ across the different ecosystems. These interrelations indicated the existence of substantial multicollinearity in the climatic and ecohydrological data matrices. 

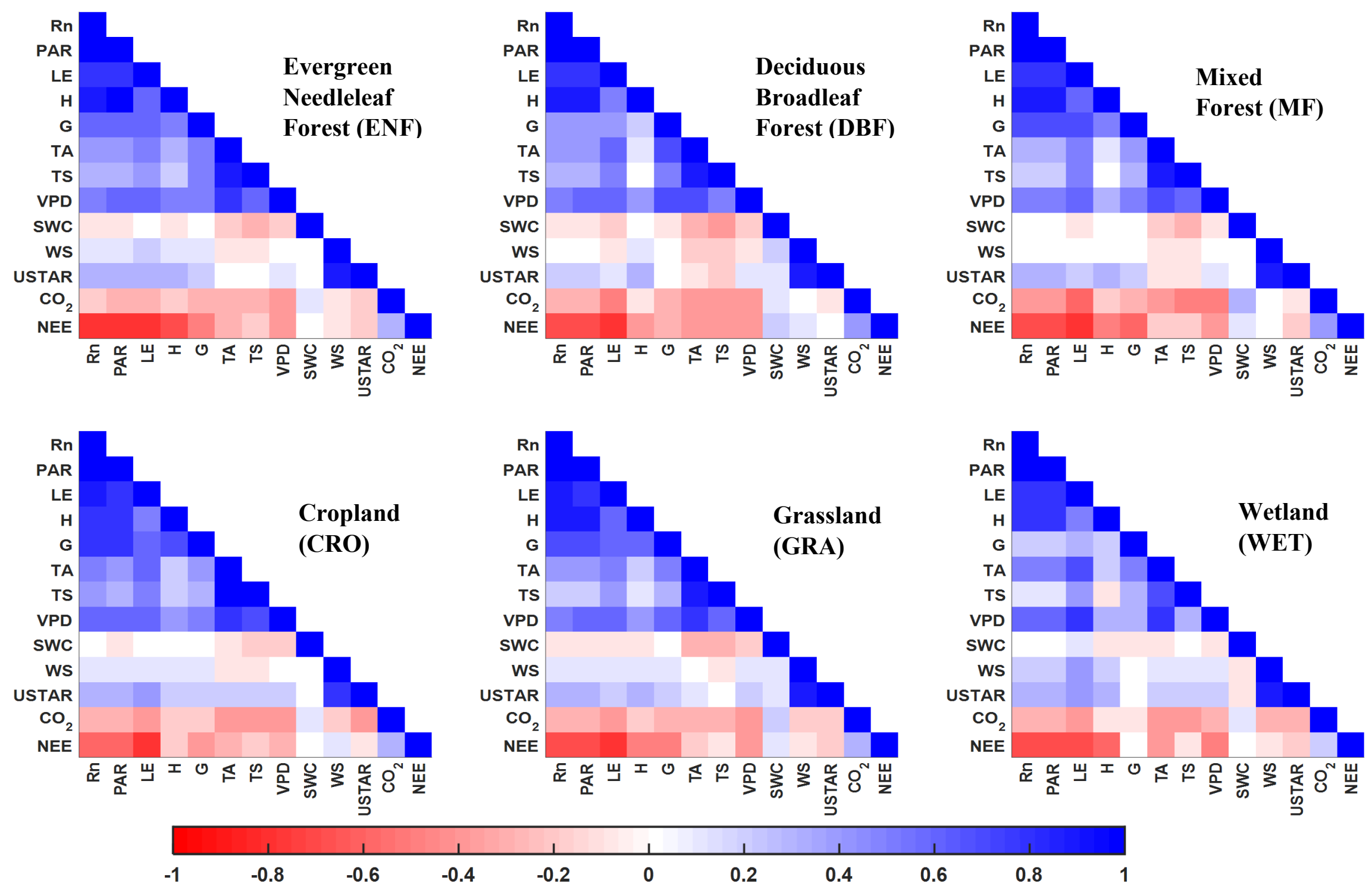

Figure 4.1: Mean correlation coefficients among NEE and climatic and ecohydrological variables for the diverse ecosystems represented in triangular maps. 


\subsection{Multivariate interrelations and grouping patterns from FA}

FA provided multivariate interrelations and grouping patterns of the participatory variables across the diverse ecosystems. By implementing the eigenvalue $\geq 1$ criteria, first three factors were extracted that maximally explained the total variance of a data matrix at each site (Figure 4.2). The data matrices combined both the predictor and response variables.

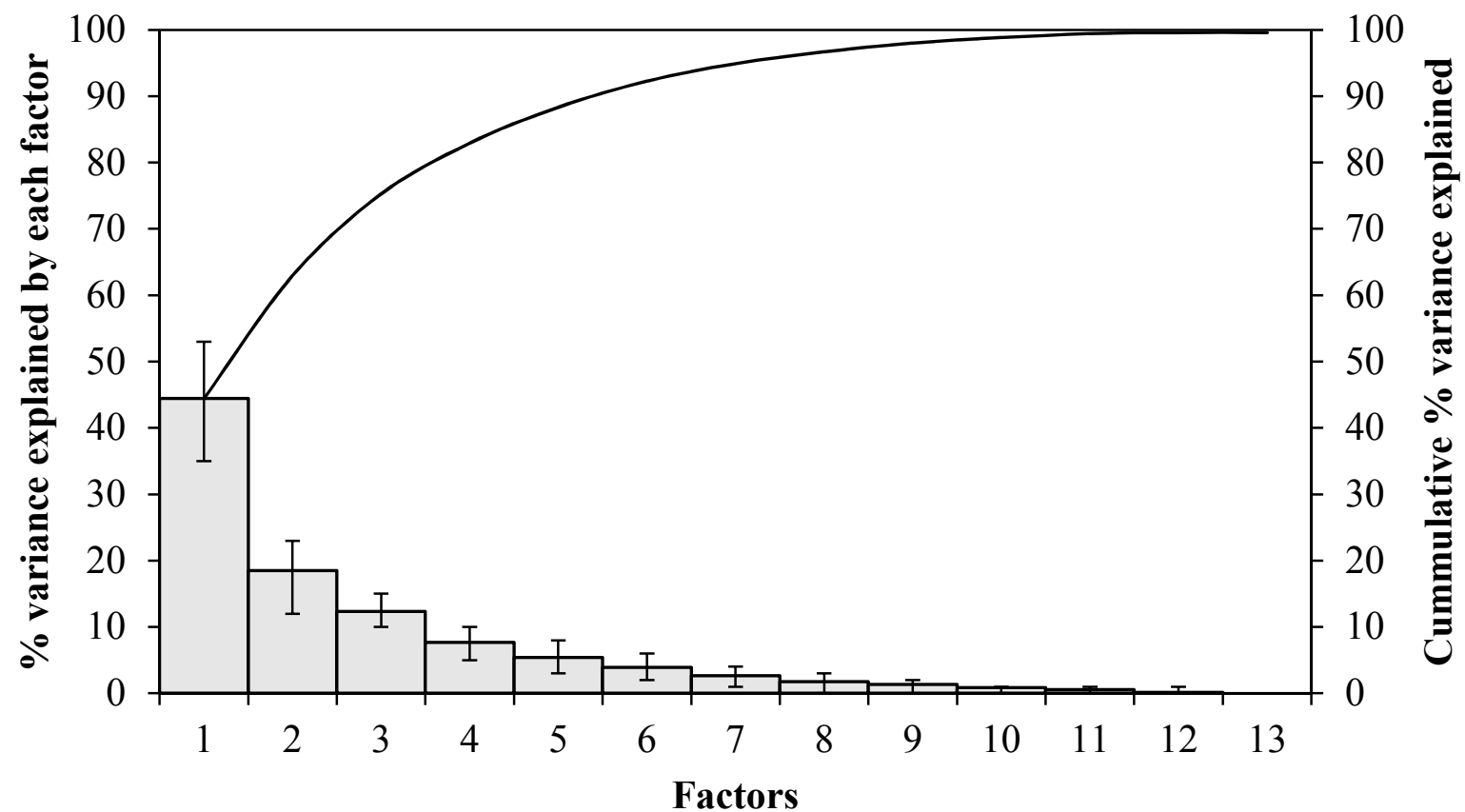

Figure 4.2: Percent variance explained by each factor for the six diverse ecosystems comprising 57 sites at the hourly scale.

The radiation, energy, and heat flux variables (Rn, PAR, LE, and $\mathrm{H}$ ) loaded highly on factor 1 (F1), temperature variables (TA, TS, and VPD) loaded highly on factor 2 (F2), and aerodynamic variables (WS and UST) loaded highly on factor 3 (F3) across the 57 sites (Figure $4.3)$. 

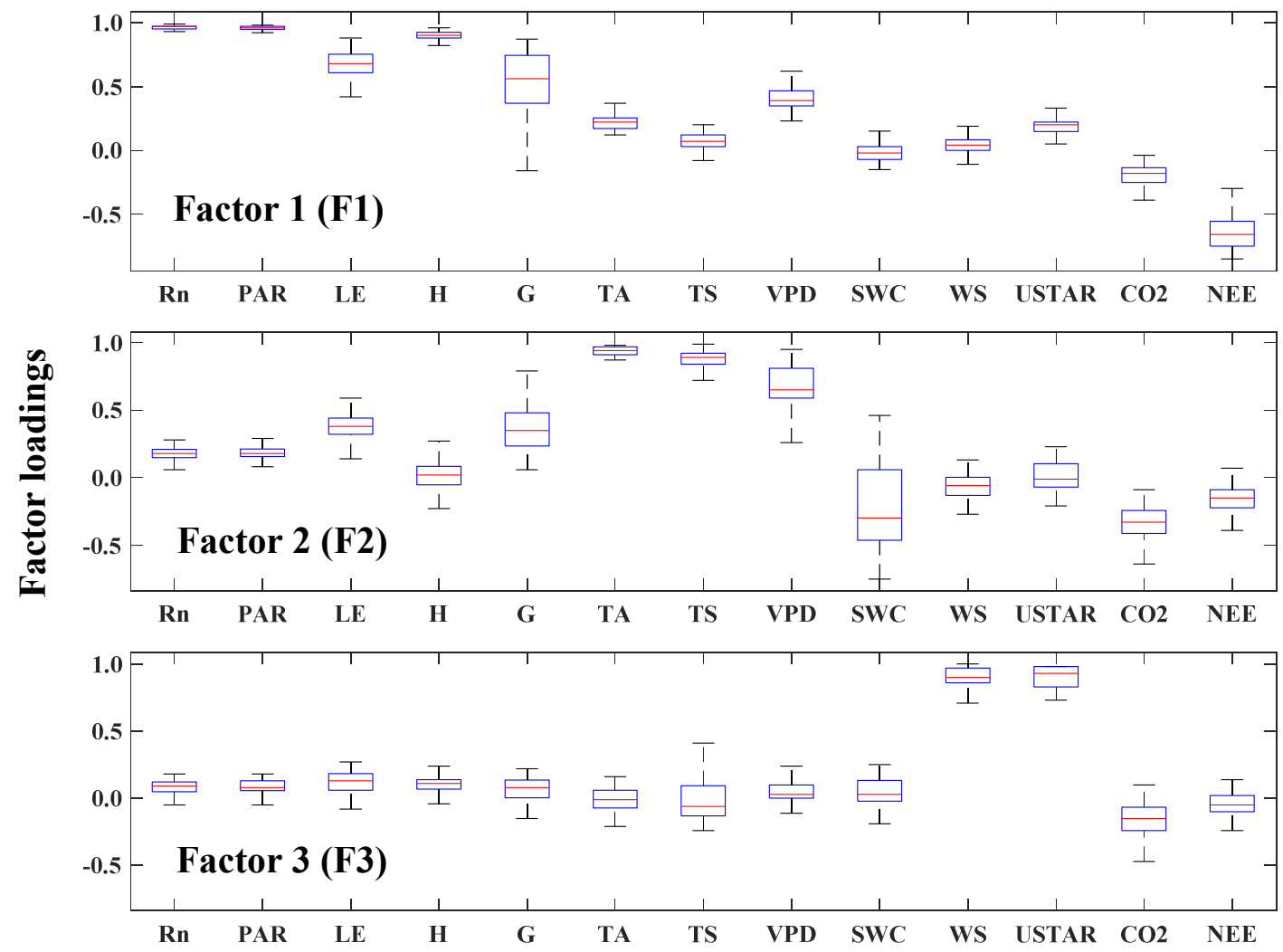

Figure 4.3: Factor loadings of the participatory variables across the 57 study sites for the first three factors.

Mean loadings of the variables under different ecosystems for the first three factors reported similar grouping patterns in all the ecosystems (Table 4.2). Both the radiation variables along with the energy and heat flux variables loaded highly (0.60 to 0.98$)$ on F1 across the different ecosystems corresponding to the highest loadings of NEE (-0.42 to -0.71$)$ among the three factors. The radiation variables represented the highest loadings ( 0.96 to 0.98$)$ on $\mathrm{F} 1$ followed by $\mathrm{H}$ that showed high loadings ranging from 0.78 to 0.92 . LE also exhibited relatively high loadings $(0.63$ to 0.73$)$. $G$ on the other hand, showed high loadings on F2 for DBF (0.68) and WET (0.48). G loaded highly ( 0.49 to 0.74$)$ on F1 for the remaining ecosystems. These loadings echoed the fact that radiation and flux variables had strong linkages with NEE and the linkage patterns were similar across different ecosystems. 
Table 4.2: Dominant hidden factors and ensemble mean loadings of the participatory variables across the diverse ecosystems.

\begin{tabular}{|c|c|c|c|c|c|c|c|c|c|c|c|c|c|c|}
\hline Ecosystems & Factor & $\mathrm{Rn}$ & PAR & $\overline{L E}$ & $\mathrm{H}$ & $\mathrm{G}$ & TA & $\mathrm{TS}$ & VPD & SWC & WS & USTAR & $\mathrm{CO}_{2}$ & $N E E$ \\
\hline \multirow{3}{*}{ ENF } & F1 & 0.96 & 0.96 & 0.69 & 0.91 & 0.49 & 0.23 & 0.11 & 0.40 & -0.02 & 0.05 & 0.20 & -0.17 & -0.71 \\
\hline & $\mathrm{F} 2$ & 0.17 & 0.18 & 0.34 & 0.08 & 0.39 & 0.92 & 0.86 & 0.66 & -0.23 & -0.04 & -0.02 & -0.30 & -0.11 \\
\hline & F3 & 0.09 & 0.09 & 0.15 & 0.11 & 0.08 & -0.03 & -0.03 & 0.04 & 0.02 & 0.92 & 0.92 & -0.14 & -0.08 \\
\hline \multirow{3}{*}{$\mathrm{DBF}$} & $\mathrm{F} 1$ & 0.96 & 0.96 & 0.63 & 0.89 & 0.23 & 0.19 & 0.09 & 0.42 & -0.05 & -0.06 & 0.20 & -0.20 & -0.56 \\
\hline & F2 & 0.20 & 0.21 & 0.45 & -0.05 & 0.68 & 0.94 & 0.90 & 0.57 & -0.33 & -0.10 & -0.06 & -0.36 & -0.32 \\
\hline & F3 & 0.05 & 0.05 & 0.01 & 0.15 & 0.06 & -0.08 & -0.15 & 0.03 & 0.13 & 0.90 & 0.95 & -0.03 & 0.06 \\
\hline \multirow{3}{*}{ MF } & $\mathrm{F} 1$ & 0.98 & 0.97 & 0.66 & 0.92 & 0.63 & 0.15 & 0.08 & 0.37 & 0.01 & -0.06 & 0.23 & -0.29 & -0.64 \\
\hline & $\mathrm{F} 2$ & 0.15 & 0.17 & 0.43 & -0.06 & 0.31 & 0.94 & 0.93 & 0.60 & -0.24 & -0.05 & -0.05 & -0.40 & -0.16 \\
\hline & F3 & 0.09 & 0.06 & 0.09 & 0.10 & 0.08 & -0.04 & -0.09 & 0.01 & 0.04 & 0.86 & 0.97 & -0.07 & -0.02 \\
\hline \multirow{3}{*}{$\mathrm{CRO}$} & F1 & 0.91 & 0.91 & 0.60 & 0.83 & 0.74 & 0.23 & 0.12 & 0.37 & 0.01 & 0.06 & 0.17 & -0.19 & -0.42 \\
\hline & $\mathrm{F} 2$ & 0.22 & 0.20 & 0.33 & 0.05 & 0.23 & 0.96 & 0.89 & 0.71 & -0.13 & -0.10 & 0.14 & -0.32 & -0.12 \\
\hline & F3 & 0.20 & 0.18 & 0.38 & 0.02 & 0.08 & 0.07 & 0.12 & 0.08 & -0.01 & 0.60 & 0.64 & -0.31 & -0.24 \\
\hline \multirow{3}{*}{ GRA } & F1 & 0.97 & 0.96 & 0.73 & 0.89 & 0.66 & 0.23 & 0.07 & 0.40 & -0.03 & 0.05 & 0.17 & -0.23 & -0.65 \\
\hline & F2 & 0.18 & 0.16 & 0.36 & 0.03 & 0.30 & 0.94 & 0.91 & 0.68 & -0.29 & -0.06 & 0.04 & -0.26 & -0.09 \\
\hline & F3 & 0.08 & 0.09 & 0.08 & 0.08 & 0.08 & 0.01 & -0.01 & 0.06 & 0.07 & 0.95 & 0.89 & -0.18 & -0.04 \\
\hline \multirow{3}{*}{ WET } & $\mathrm{F} 1$ & 0.97 & 0.96 & 0.64 & 0.78 & 0.14 & 0.28 & -0.04 & 0.47 & 0.04 & 0.12 & 0.14 & -0.16 & -0.66 \\
\hline & $\mathrm{F} 2$ & 0.17 & 0.17 & 0.44 & -0.06 & 0.48 & 0.81 & 0.74 & 0.50 & -0.06 & 0.13 & 0.19 & -0.39 & -0.14 \\
\hline & F3 & 0.13 & 0.13 & 0.33 & 0.13 & -0.01 & 0.18 & 0.14 & 0.21 & -0.06 & 0.76 & 0.77 & -0.27 & -0.12 \\
\hline
\end{tabular}

Notes: F1-3 refer to three different factors. Bold indicates variables having moderate to high loadings on factors. The ecosystem-specific mean loadings were obtained by averaging the site-specific loadings over each ecosystem type. ENF, DBF, MF, CRO, GRA, and WET refer to evergreen needleleaf forests, deciduous broadleaf forests, mixed forests, grasslands, croplands, and wetlands, respectively. 
The loadings were consistently high $(0.74$ to 0.96$)$ for the temperature variables (TA and TS) on F2 that correspondingly exhibited low to moderate loadings (-0.09 to -0.32) of NEE in all the ecosystems. F2 also represented the high factor loadings ( 0.50 to 0.71$)$ of VPD along with the low to moderate loadings $(-0.06$ to -0.33$)$ of SWC. Overall, the loadings of the temperature and hydrologic variables (TA, TS, VPD, and SWC) with respect to the loadings of NEE depicted moderate linkages and the patterns were consistent across the different ecosystems. On F3, the loadings of NEE were low (-0.24 to 0.06$)$ corresponding to the moderate to high loadings of the aerodynamic variables (WS and USTAR; 0.60 to 0.97 ), which were consistent across the six ecosystems. The loadings of ambient atmospheric $\mathrm{CO}_{2}$ concentration was loaded weakly to moderately (-0.03 to -0.40$)$ on all the factors representing moderate linkages with NEE across all the ecosystems.

\subsection{Formation of bio-physical process components}

The results from FA identified similar interrelations and grouping patterns of the climatic and ecohydrological variables towards NEE across the diverse ecosystems. The loadings from FA for the 57 sites (Figure 4.3) along with the ensemble mean loadings at the six ecosystems (Table 4.2) clearly illustrated that the cluster of radiation, energy, and heat flux variables (Rn, PAR, LE, H, and $\mathrm{G}$ ) formed the 'radiation-energy (RE)' component and was the most dominant biophysical process component driving NEE across all the ecosystems. Even though for some ecosystems G was outside the cluster of radiation, energy, and heat flux variables (see DBF and WET in Table 4.2), considering the energy balance closure, G was kept in the RE component. The factor loadings further portrayed the cluster of the temperature and hydrologic variables in the same group consistently across the diverse ecosystems corresponding to their moderate linkages with NEE. Considering the linkages of hydrologic processes and their close associations with the temperature 
variables, the 'temperature-hydrology (TH)' biophysical process component was formed consisting of TA, TS, VPD, and SWC (Table 4.2). The aerodynamic variables (WS and USTAR) clustered in another group separate from the RE and $\mathrm{TH}$ components. Based on this, the 'aerodynamic (AD)' component was defined that showed the weakest linkages with NEE among all the biophysical process components. The atmospheric ambient $\mathrm{CO}_{2}$ concentration exhibited moderate linkages with NEE separately from the other clusters. Based on this, the 'ambient atmospheric $\mathrm{CO}_{2}(\mathrm{AC})^{\prime}$ component was formed. Since the $\beta$ of the variables exhibited both negative and positive values, aggregating them by addition would result in lumped values providing misleading linkages with NEE. Therefore, $\beta$ of the variables under each component were aggregated through vector summations to obtain the magnitude of the component linkages. Aggregated linkages of these components were computed using the following equations:

$$
\begin{aligned}
& \beta_{R E}=\sqrt{\beta_{R n}^{2}+\beta_{P A R}^{2}+\beta_{L E}^{2}+\beta_{H}^{2}+\beta_{G}^{2}} \\
& \beta_{T H}=\sqrt{\beta_{T A}^{2}+\beta_{T S}^{2}+\beta_{V P D}^{2}+\beta_{S W C}^{2}} \\
& \beta_{A D}=\sqrt{\beta_{W S}^{2}+\beta_{U S T A R}^{2}} \\
& \beta_{A C}=\sqrt{\beta_{C O 2}^{2}}
\end{aligned}
$$

\subsection{Estimation of the linkages across diverse ecosystems}

The three-fold criterion based synthesis resulted in 3 to 4 PLS components representing the minimum number of components that demonstrated optimal PLSR models of NEE across the different ecosystems (Figure 4.4). 
(a)

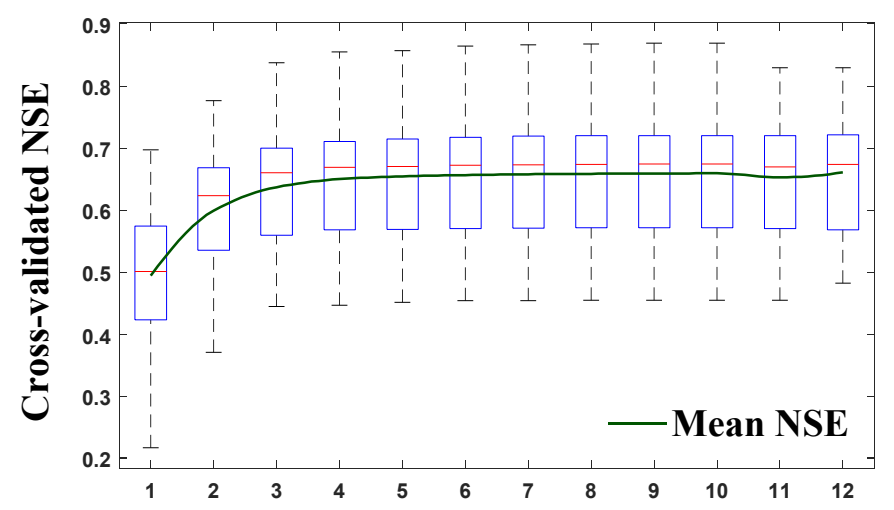

(b)

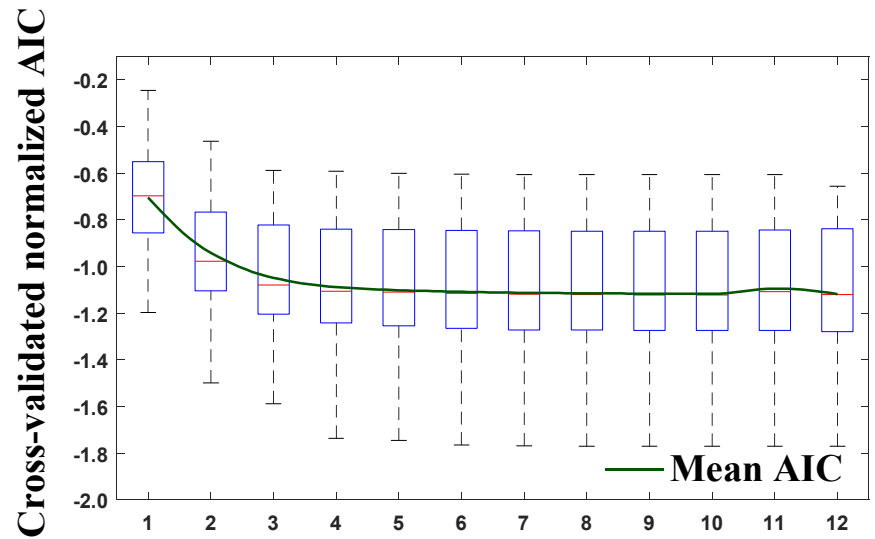

(c)

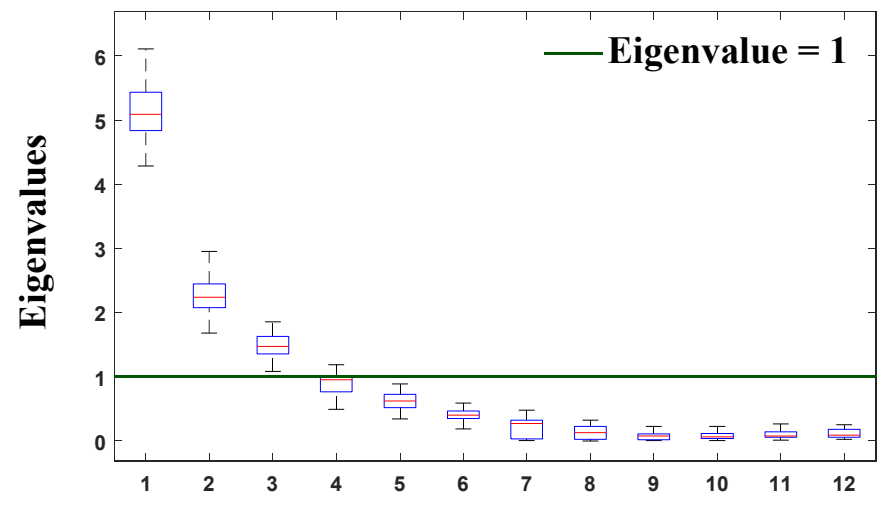

Number of partial least squares (PLS) components

Figure 4.4: Boxplots of cross-validated (a) NSE of NEE, (b) normalized AIC, and (c) eigenvalues of the data matrix with the number of partial least squares (PLS) components across the 57 sites.

LE exhibited the highest estimation of $\beta$ across all the ecosystems except for WET where the estimations of both LE and H were similar (Figure 4.5). 

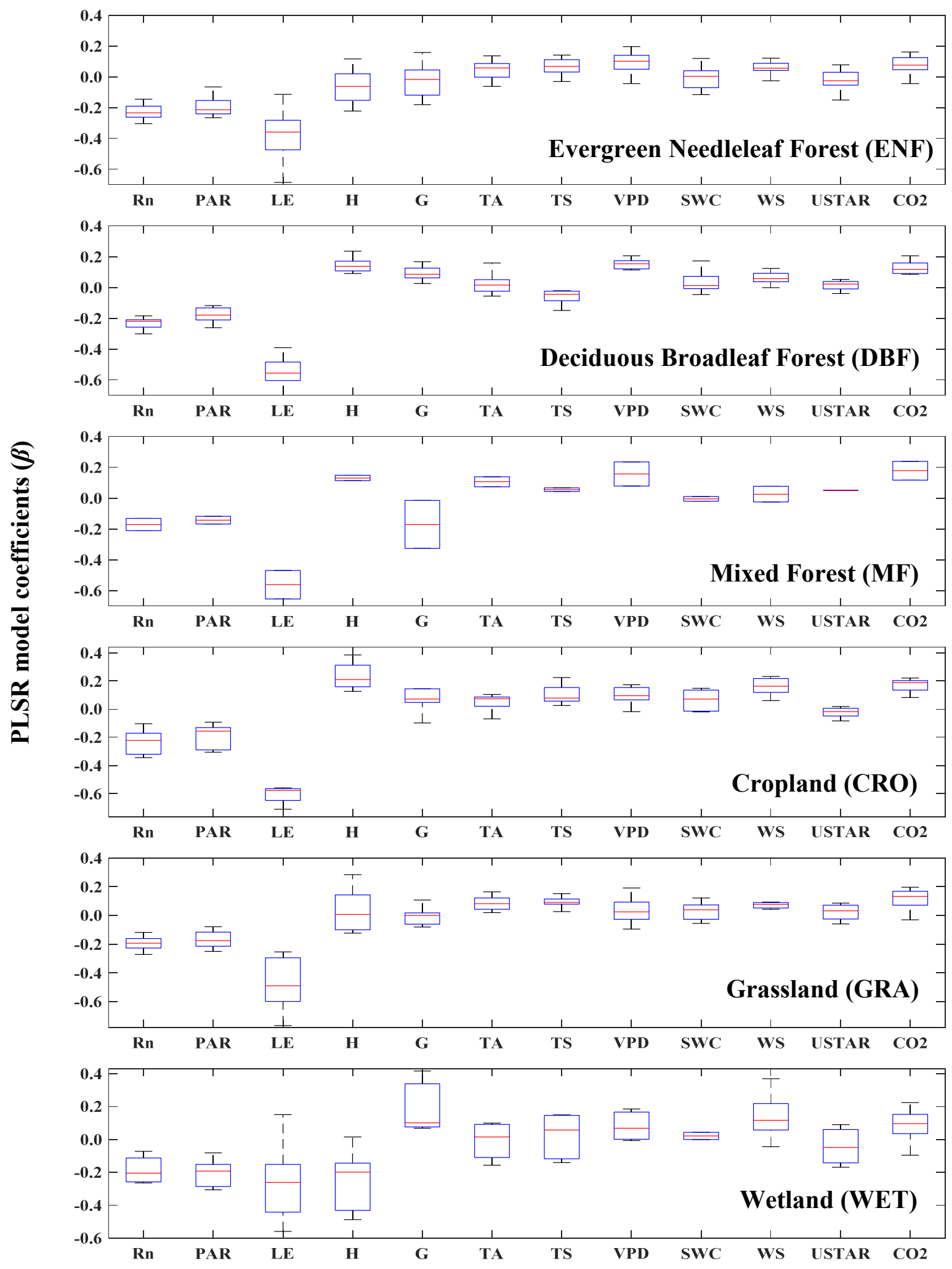

Figure 4.5: Summary of the estimated PLSR model coefficients $(\beta)$ for the diverse ecosystems. 
The ecosystem-specific fitting efficiencies (NSE) and accuracies (RSR) obtained from the 1000 Monte-Carlo estimations of the optimized PLSR models were within acceptable ranges (NSE $=0.60$ to 0.68 and $\mathrm{RSR}=0.56$ to 0.63 ) (Table 4.3). Models developed using $\beta$ from 1000 MonteCarlo estimations exhibited good predictions for all the 57 sites (see Figure A of Appendices).The ecosystem-specific ensemble mean of the estimated $\beta$ determined were similar across the six ecosystems and their respective standard deviations showed low parameter uncertainty (Table 4.3).

All the variables under the 'radiation-energy (RE)' component exhibited higher values of $\beta$ depicting their greater linkages with NEE. Among the variables under the RE component, LE was identified to be the strongest predictor of NEE across all the ecosystems with its highest value of $\beta(-0.25$ to -0.59$)$. Only in the WET, the linkage of LE was moderate (-0.25) compared to the higher values in the other ecosystems and was further similar to the $\beta$ of $\mathrm{H}(-0.24)$. The signs of the $\beta$ for Rn, PAR, and LE in all the ecosystems were negative exhibiting their influence on $\mathrm{CO}_{2}$ uptake. $\mathrm{H}$ and $\mathrm{G}$ on the other hand, showed mixed signs that referred to both $\mathrm{CO}_{2}$ uptake and release. The variables in the 'temperature-hydrology (TH)' component exhibited both low and moderate linkages. Low values of $\beta$ were mostly observed for SWC (-0.01 to 0.06$)$ and TA ( -0.01 to 0.11 ) whereas TS (-0.06 to 0.11$)$ and VPD ( 0.03 to 0.16 ) showed relatively moderate linkages. The signs $\beta$ in the TH component were positive referring to their dominance on driving $\mathrm{CO}_{2}$ release. 
Table 4.3: Ensemble means and standard deviations (in parenthesis) of the Z-score PLSR model coefficients $(\beta)$, representing the relative linkages of NEE under different ecosystem types. The ecosystem type-specific statistics were computed from the corresponding site-specific $\beta$, as obtained from 1000 Monte-Carlo estimations.

\begin{tabular}{|c|c|c|c|c|c|c|}
\hline Variables & ENF & $\mathrm{DBF}$ & $\mathrm{MF}$ & $\mathrm{CRO}$ & GRA & WET \\
\hline $\mathrm{Rn}$ & $-0.23(0.005)$ & $-0.23(0.004)$ & $-0.17(0.006)$ & $-0.23(0.005)$ & $-0.19(0.005)$ & $-0.19(0.005)$ \\
\hline PAR & $-0.20(0.004)$ & $-0.18(0.004)$ & $-0.14(0.005)$ & $-0.19(0.004)$ & $-0.17(0.003)$ & $-0.20(0.005)$ \\
\hline LE & $-0.38(0.004)$ & $-0.55(0.004)$ & $-0.56(0.006)$ & $-0.59(0.004)$ & $-0.47(0.004)$ & $-0.25(0.005)$ \\
\hline $\mathrm{H}$ & $-0.07(0.010)$ & $0.15(0.011)$ & $0.13(0.012)$ & $0.23(0.010)$ & $0.03(0.011)$ & $-0.24(0.010)$ \\
\hline $\mathrm{G}$ & $-0.05(0.004)$ & $0.09(0.005)$ & $-0.17(0.009)$ & $0.10(0.007)$ & $-0.01(0.006)$ & $0.19(0.010)$ \\
\hline TA & $0.04(0.007)$ & $0.02(0.007)$ & $0.11(0.011)$ & $0.05(0.008)$ & $0.08(0.008)$ & $-0.01(0.008)$ \\
\hline $\mathrm{TS}$ & $0.07(0.004)$ & $-0.06(0.004)$ & $0.06(0.005)$ & $0.11(0.004)$ & $0.09(0.005)$ & $0.02(0.006)$ \\
\hline VPD & $0.09(0.006)$ & $0.14(0.005)$ & $0.16(0.007)$ & $0.10(0.005)$ & $0.03(0.005)$ & $0.08(0.007)$ \\
\hline SWC & $-0.01(0.007)$ & $0.04(0.006)$ & $0.01(0.011)$ & $0.06(0.010)$ & $0.03(0.007)$ & $0.02(0.010)$ \\
\hline WS & $0.06(0.007)$ & $0.06(0.007)$ & $0.03(0.009)$ & $0.16(0.007)$ & $0.07(0.007)$ & $0.14(0.007)$ \\
\hline USTAR & $-0.01(0.005)$ & $0.02(0.006)$ & $0.05(0.007)$ & $-0.02(0.006)$ & $0.02(0.005)$ & $-0.04(0.007)$ \\
\hline $\mathrm{CO}_{2}$ & $0.07(0.005)$ & $0.14(0.006)$ & $0.18(0.007)$ & $0.17(0.006)$ & $0.11(0.005)$ & $0.08(0.007)$ \\
\hline $\begin{array}{l}\text { Optimum PLS } \\
\text { components }\end{array}$ & 3 to 4 & 3 & 3 to 4 & 3 to 4 & 3 to 4 & 3 to 4 \\
\hline NSE & 0.64 & 0.68 & 0.66 & 0.63 & 0.60 & 0.63 \\
\hline RSR & 0.59 & 0.56 & 0.58 & 0.60 & 0.63 & 0.60 \\
\hline
\end{tabular}

Notes: NSE refers to the model fitting efficiency, whereas RSR refers to the accuracy of model predictions. The ecosystem-specific mean NSE and RSR are reported here. ENF, DBF, MF, CRO, GRA, and WET refer to evergreen needleleaf forests, deciduous broadleaf forests, mixed forests, grasslands, croplands, and wetlands, respectively. 
In general, both the aerodynamic variables (WS and USTAR) under the 'aerodynamic (AD)' component exhibited low values of $\beta$ across all the ecosystems. The linkages of WS at the CRO and WET ecosystems were comparatively moderate (0.14 to 0.16 ) compared to the other ecosystems that indicated relatively strong influences of aerodynamic processes on driving NEE at these low canopy ecosystems. The $\beta$ of the ambient atmospheric $\mathrm{CO}_{2}$ concentration under the 'ambient atmospheric $\mathrm{CO}_{2}(\mathrm{AC})$ ' component portrayed a rather moderate magnitude ( 0.07 to 0.17$)$ that indicated moderate linkages with NEE. The signs of $\beta$ for $\mathrm{CO}_{2}$ concentration referred to its influence on carbon release across all the ecosystems.

Linkages of the four biophysical process components were quantified from the estimated Z-score PLSR model $\beta$ using the equations 4.1-4.4 (Figure 4.6).

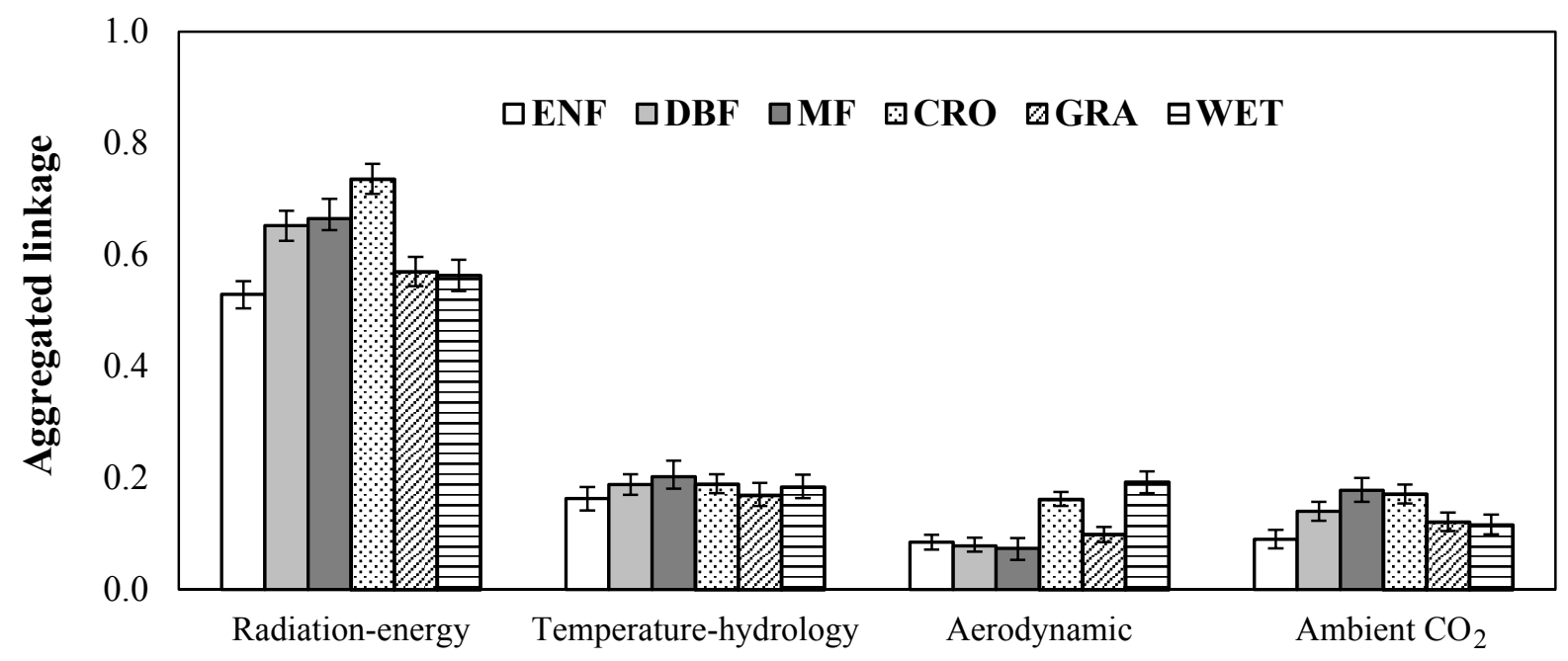

Figure 4.6: Aggregated linkages of NEE with the major biophysical process components across different ecosystems. ENF, DBF, MF, CRO, GRA, and WET refer to evergreen needleleaf forests, deciduous broadleaf forests, mixed forests, croplands, grasslands, and wetlands, respectively. Error bars represent $95 \%$ upper and lower confidence limits of the aggregated linkages. 
The uncertainties of the aggregated linkages were determined by the upper and lower $95 \%$ confidence limits using the standard deviations of $\beta$ obtained from the 1000 Monte-Carlo approximations. From the calculated uncertainty, it was evident that hourly linkages of NEE remained stable across the diverse ecosystems. The quantified aggregated linkages illustrated that the RE component was by far the most dominant process component driving NEE irrespective of ecosystem types. The TH component acted as a moderate process component that was also consistent across the different ecosystems. The $\mathrm{AD}$ component was found to be relatively stronger for the low canopy ecosystem types (i.e., CRO, GRA and WET) compared to the high canopy ecosystems (i.e., ENF, DBF, and MF). The 'ambient $\mathrm{CO}_{2}$ ' also acted as a moderate process component that drove NEE. 


\section{CHAPTER 5: RESULTS OF MULTI-TEMPORAL LINKAGES OF NEE AT THE EVERGLADES SHORT HYDRO-PERIOD SITE}

\subsection{Linear relationships from correlation analysis}

The Pearson's correlation coefficients between NEE and the climatic and ecohydrological variables were investigated for the four temporal scales at the Everglades short hydro-period site. The radiation variables ( $\mathrm{Rn}$ and PAR) showed moderate to strong correlations $(r=-0.50$ to -0.60$)$ with NEE over the different temporal scales (Table 5.1). Among the energy and heat flux variables, $\mathrm{H}$ exhibited the strongest correlations ( $r=-0.70$ to -0.77$)$ with NEE, whereas LE and G showed weak to moderate interrelations $(r=-0.29$ to -0.49$)$. $\mathrm{H}$ also represented the strongest correlations with NEE among all the variables across the temporal scales. Both TA and VPD showed weak to moderate interrelations ( $r=-0.28$ to -0.52$)$ with NEE, TS on the contrary showed weak correlations $(r=-0.08$ to -0.10$)$ with NEE. NEE exhibited weak to moderate correlations with the hydrologic (WL and SWC; $r=0.23$ to 0.51 ) variables. The aerodynamic variables (WS and USTAR) and $\mathrm{CO}_{2}$ also showed weak to moderate interrelations (WS; $r=0.10$ to 0.25 , USTAR; $r=-0.34$ to 0.08 , and $\mathrm{CO}_{2} ; r=-0.14$ to -0.29$)$ with NEE at the different temporal scales.

Table 5.1: Pearson's correlation coefficients $(r)$ between NEE and climatic and ecohydrological variables at the different temporal scales.

\begin{tabular}{llllllllllllll}
\hline $\begin{array}{l}\text { Time } \\
\text { scale }\end{array}$ & Rn & PAR & LE & $\mathrm{H}$ & $\mathrm{G}$ & $\mathrm{TA}$ & $\mathrm{TS}$ & $\mathrm{VPD}$ & WL & SWC & WS & USTAR & $\mathrm{CO}_{2}$ \\
\hline 1-day & -0.51 & -0.50 & -0.49 & -0.70 & -0.29 & -0.28 & -0.10 & -0.35 & 0.33 & 0.23 & 0.25 & 0.08 & -0.14 \\
8-day & -0.53 & -0.51 & -0.43 & -0.72 & -0.33 & -0.29 & $-0.10^{*}$ & -0.39 & 0.43 & 0.32 & 0.25 & $-0.08^{*}$ & -0.29 \\
15-day & -0.57 & -0.55 & -0.39 & -0.75 & -0.36 & -0.30 & $-0.10^{*}$ & -0.45 & 0.46 & 0.34 & $0.15^{*}$ & -0.20 & -0.25 \\
30-day & -0.60 & -0.58 & -0.37 & -0.77 & -0.38 & -0.29 & $-0.08^{*}$ & -0.52 & 0.51 & 0.38 & $0.10^{*}$ & -0.34 & -0.28 \\
\hline
\end{tabular}

Notes: '*' refers to the correlations that were not statistically significant ( $\mathrm{p}$-value $>0.05$ ). 
The Pearson correlation matrix gave information on the multicollinear structure of the participatory variables (Figure 5.1). The radiation variables (Rn and PAR) were strongly interrelated ( $r=0.97$ to 0.99 ) across the four temporal scales.
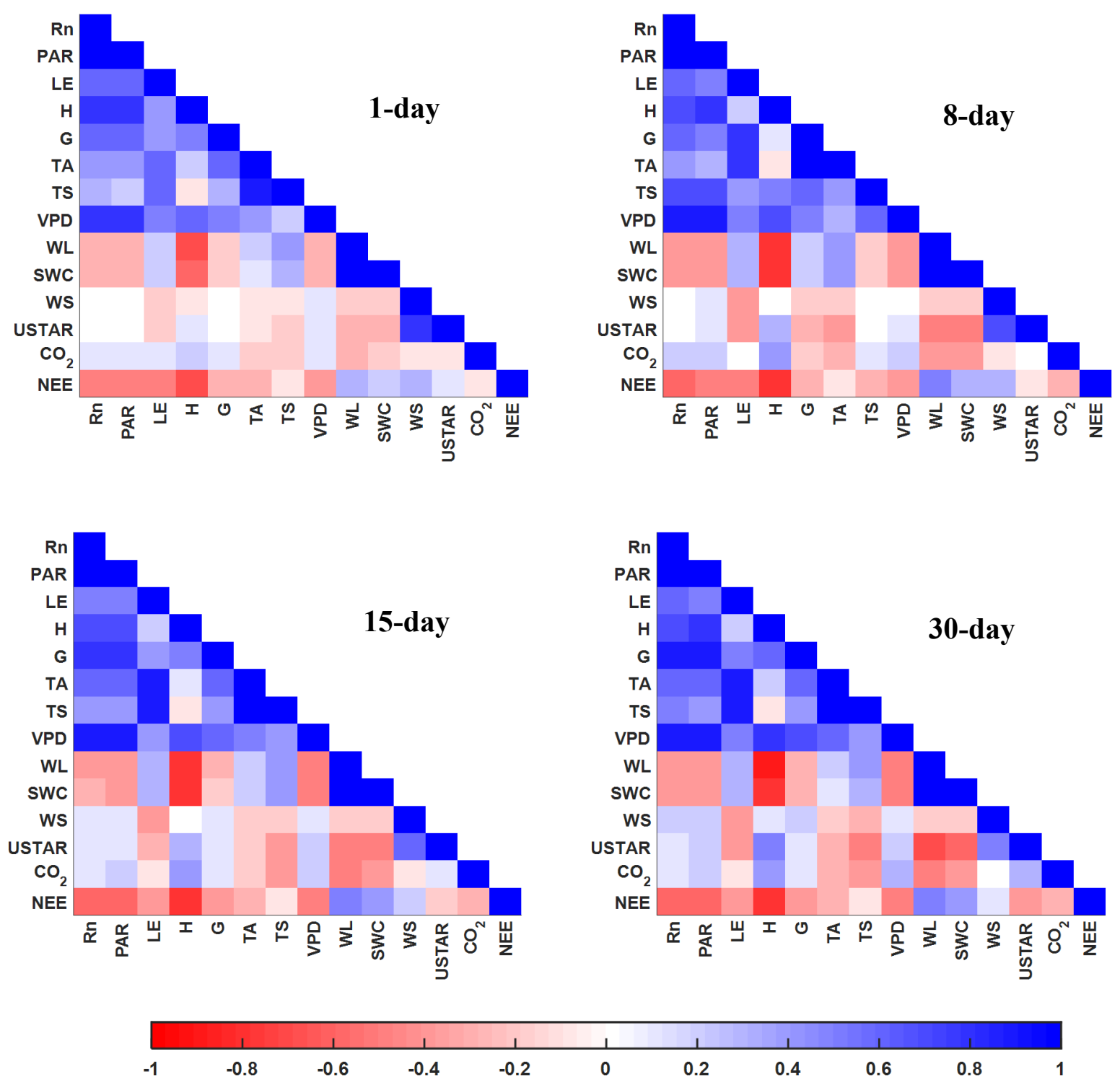

Figure 5.1: Triangular maps representing correlation coefficients of NEE, climatic, and ecohydrological variables for the different temporal scales.

Similar strong interrelations were also exhibited between the hydrologic (WL and SWC; $r$ $=0.93$ to 0.94 ) and the temperature variables (TA and TS; $r=0.93$ to 0.94 ). Although, the energy 
and heat flux variables (LE, $\mathrm{H}$, and $\mathrm{G}$ ) showed moderate to strong interrelations $(r=0.46$ to 0.83 ) with the radiation variables, the correlations among themselves were weak to moderate $(r=0.15$ to 0.54$)$ over the different temporal scales. VPD showed weak to moderate interrelations $(r=0.24$ to 0.55 ) with the temperature variables. The aerodynamic variables (WS and USTAR) showed moderate $(r=0.46$ to 0.79$)$ interrelations across the temporal scales. Other significant interrelations over the different temporal scales were between VPD and the radiation variables $(r$ $=0.80$ to 0.89$), \mathrm{LE}$ and the temperature variables $(r=0.55$ to 0.86$), \mathrm{H}$ and the hydrologic variables $(r=-0.62$ to -0.82$)$.

\subsection{Multivariate interrelations and grouping patterns from FA}

Multivariate interrelations among NEE and the climatic and ecohydrological variables were obtained through FA at each temporal scale. The eigenvalue criteria resulted in three independent latent factors at each temporal scale that optimally explained the total data variance. The three factors explained $73-82 \%$ of the total data variance across the temporal scales (Table 5.2).

Table 5.2: Percent variance explained by each factor at the different temporal scales for the Florida Everglades short hydro-period site.

\begin{tabular}{lllll}
\hline Factor & 1-day & 8 -day & 15-day & 30 -day \\
\hline 1 & 37 & 39 & 40 & 43 \\
2 & 23 & 27 & 28 & 30 \\
3 & 13 & 11 & 11 & 9 \\
4 & 7 & 5 & 5 & 5 \\
5 & 6 & 5 & 5 & 4 \\
6 & 5 & 4 & 4 & 3 \\
7 & 4 & 3 & 3 & 2 \\
8 & 2 & 2 & 2 & 2 \\
9 & 2 & 1 & 1 & 1 \\
10 & 1 & 1 & 1 & 1 \\
11 & 1 & 1 & 0 & 0 \\
12 & 0 & 0 & 0 & 0 \\
13 & 0 & 0 & 0 & 0 \\
\hline
\end{tabular}


Both the radiation variables loaded highly $(0.92$ to 0.97$)$ on factor 1 (F1) that corresponded to moderate linkages of NEE (-0.46 to -0.50$)$ across the different temporal scales (Table 5.3). Although the two energy and heat flux variables, $\mathrm{H}$ and $\mathrm{G}$ loaded between 0.47 to 0.77 on $\mathrm{F} 1, \mathrm{H}$ additionally loaded moderately to highly $(-0.51$ to -0.69$)$ on factor 2 (F2). F2 was further highly loaded $(0.89$ to 0.97$)$ with the hydrologic variables coupled with the weak to moderate $(0.28$ to 0.43) loadings of NEE. VPD consistently loaded highly (0.75 to 0.77 ) on F1 over the four temporal scales. The remaining flux variable LE, loaded moderately (0.56) on F1 at 1-day scale and loaded highly ( 0.67 to 0.76$)$ on factor 3 (F3) on the remaining temporal scales. NEE loaded weakly $(-0.10$ to -0.20$)$ on $\mathrm{F} 3$ accompanied by the high loadings $(0.85$ to 0.97$)$ of the temperature variables in all the temporal scales. 
Table 5.3: Dominant latent factors and optimized loadings of NEE, climatic, and ecohydrological variables at the different temporal scales.

\begin{tabular}{llrrrrrrrrrrrrrr}
\hline $\begin{array}{l}\text { Temporal } \\
\text { scales }\end{array}$ & Variables & Rn & PAR & LE & H & G & TA & TS & VPD & WL & SWC & WS & USTAR & CO 2 & $N E E$ \\
\hline \multirow{4}{*}{ 1-day } & F1 & $\mathbf{0 . 9 5}$ & $\mathbf{0 . 9 7}$ & $\mathbf{0 . 5 6}$ & $\mathbf{0 . 7 0}$ & 0.47 & 0.25 & 0.14 & $\mathbf{0 . 7 7}$ & -0.18 & -0.17 & -0.02 & -0.04 & 0.10 & $\mathbf{- 0 . 4 6}$ \\
& F2 & -0.13 & -0.13 & 0.23 & $\mathbf{- 0 . 5 1}$ & -0.20 & 0.06 & 0.31 & -0.18 & $\mathbf{0 . 9 7}$ & $\mathbf{0 . 9 1}$ & -0.18 & -0.33 & -0.22 & 0.28 \\
& F3 & 0.20 & 0.16 & 0.42 & 0.01 & $\mathbf{0 . 5 0}$ & $\mathbf{0 . 9 7}$ & $\mathbf{0 . 8 5}$ & 0.22 & 0.14 & 0.11 & -0.13 & -0.11 & -0.17 & -0.19 \\
& F1 & $\mathbf{0 . 9 3}$ & $\mathbf{0 . 9 5}$ & 0.42 & $\mathbf{0 . 6 4}$ & $\mathbf{0 . 5 9}$ & 0.30 & 0.19 & $\mathbf{0 . 7 7}$ & -0.26 & -0.23 & 0.08 & 0.04 & 0.17 & $\mathbf{- 0 . 4 1}$ \\
8-day & F2 & -0.19 & -0.20 & 0.24 & $\mathbf{- 0 . 6 3}$ & -0.19 & 0.08 & 0.28 & -0.29 & $\mathbf{0 . 9 4}$ & $\mathbf{0 . 8 9}$ & -0.13 & -0.45 & -0.33 & 0.39 \\
& F3 & 0.28 & 0.22 & $\mathbf{0 . 6 7}$ & -0.03 & 0.41 & $\mathbf{0 . 9 4}$ & $\mathbf{0 . 9 0}$ & 0.26 & 0.22 & 0.17 & -0.23 & -0.29 & -0.23 & -0.20 \\
& F1 & $\mathbf{0 . 9 2}$ & $\mathbf{0 . 9 4}$ & 0.34 & $\mathbf{0 . 5 9}$ & $\mathbf{0 . 7 0}$ & 0.36 & 0.22 & $\mathbf{0 . 7 5}$ & -0.26 & -0.21 & 0.15 & 0.08 & 0.15 & $\mathbf{- 0 . 4 6}$ \\
15-day & F2 & -0.20 & -0.23 & 0.20 & $\mathbf{- 0 . 6 7}$ & -0.15 & 0.08 & 0.27 & -0.36 & $\mathbf{0 . 9 3}$ & $\mathbf{0 . 9 0}$ & -0.09 & -0.47 & -0.36 & 0.40 \\
& F3 & 0.30 & 0.25 & $\mathbf{0 . 7 5}$ & -0.04 & 0.31 & $\mathbf{0 . 9 0}$ & $\mathbf{0 . 9 3}$ & 0.30 & 0.25 & 0.17 & -0.26 & -0.25 & -0.30 & -0.14 \\
& F1 & $\mathbf{0 . 9 2}$ & $\mathbf{0 . 9 3}$ & 0.39 & $\mathbf{0 . 6 2}$ & $\mathbf{0 . 7 7}$ & 0.39 & 0.22 & $\mathbf{0 . 7 5}$ & -0.27 & -0.21 & 0.24 & 0.12 & 0.17 & $\mathbf{- 0 . 5 0}$ \\
30-day & F2 & -0.22 & -0.25 & 0.21 & $\mathbf{- 0 . 6 9}$ & -0.14 & 0.07 & 0.25 & -0.42 & $\mathbf{0 . 9 2}$ & $\mathbf{0 . 9 1}$ & -0.05 & $\mathbf{- 0 . 5 6}$ & -0.39 & 0.43 \\
& F3 & 0.31 & 0.25 & $\mathbf{0 . 7 6}$ & -0.04 & 0.28 & $\mathbf{0 . 9 0}$ & $\mathbf{0 . 9 4}$ & 0.34 & 0.26 & 0.15 & -0.30 & -0.34 & -0.36 & -0.10 \\
\hline
\end{tabular}

Notes: F-1 to 3 refer to the three optimal factors. Bold indicates variables having moderate to high loadings on factors. 


\subsection{Formation of bio-physical process components}

The results obtained from FA provided similar multi-temporal interrelations and grouping patterns for the climatic and ecohydrological variables. A few exceptions were exhibited by LE and H. LE grouped with the radiation variables at 1-day scale and then with the temperature variables at the higher temporal scales. This contribution of LE with the temperature variables at the higher temporal scales was reflected in the correlation matrix (Figure 5.1). H grouped with both the radiation and the hydrologic variables, which was also illustrated in the correlation matrix (Figure 5.1). Although the grouping patterns were relatively consistent across the different temporal scales, they were not entirely similar to the grouping patterns found in the hourly scale analysis. The biophysical process components in the multi-temporal linkage analysis were still formed according to the groups in the hourly scale analysis to compare the linkages between the spatial and temporal scales. Through literature review, it was found that WL was an important driver of $\mathrm{CO}_{2}$ flux in the Everglades short hydro-period site. Therefore, WL was included in the TH component. Aggregated linkages of the components were computed using the following equations:

$$
\begin{aligned}
& \beta_{R E}=\sqrt{\beta_{R n}^{2}+\beta_{P A R}^{2}+\beta_{L E}^{2}+\beta_{H}^{2}+\beta_{G}^{2}} \\
& \beta_{T H}=\sqrt{\beta_{T A}^{2}+\beta_{T S}^{2}+\beta_{V P D}^{2}+\beta_{S W C}^{2}+\beta_{W L}^{2}} \\
& \beta_{A D}=\sqrt{\beta_{W S}^{2}+\beta_{U S T A R}^{2}} \\
& \beta_{A C}=\sqrt{\beta_{C O 2}^{2}}
\end{aligned}
$$

\subsection{Estimation of the multi-temporal linkages at the Florida Everglades short hydro-period}

Optimum Z-score PLSR models of NEE for the 13 climatic and ecohydrological drivers were attained using three PLS components in all the temporal scales based on the eigenvalue criteria 
(Table 5.4). Although the three-fold criterion based synthesis was successful in identifying the minimum number of PLS components to obtain optimal PLSR model in hourly linkage analysis, for the multi-temporal linkage analysis only the eigenvalue $\geq 1$ criteria succeeded in this purpose. During the analysis it was observed that at the aggregated temporal scales, eigenvalue $\geq 1$ criteria was more successful to capture the total system variance of the data matrix and therefore resulted in meaningful linkages compared to the maximum NSE and minimum AIC criterion. Therefore, only eigenvalue $\geq 1$ criteria was used in the multi-temporal linkage analysis at the Everglades short hydro-period marsh site to find the number of PLS components for optimal PLSR models at each of the temporal scales. The performances of the PLSR models were good for all the four temporal scales $(\mathrm{NSE}=0.62$ to 0.73 and $\mathrm{RSR}=0.51$ to 0.61$)$. According to Moriasi, et al., 2007, the estimated PLSR models could be characterized as good to satisfactory. The models developed using estimated $\beta$ exhibited good predictions in all the temporal scales (Figure 5.2). 

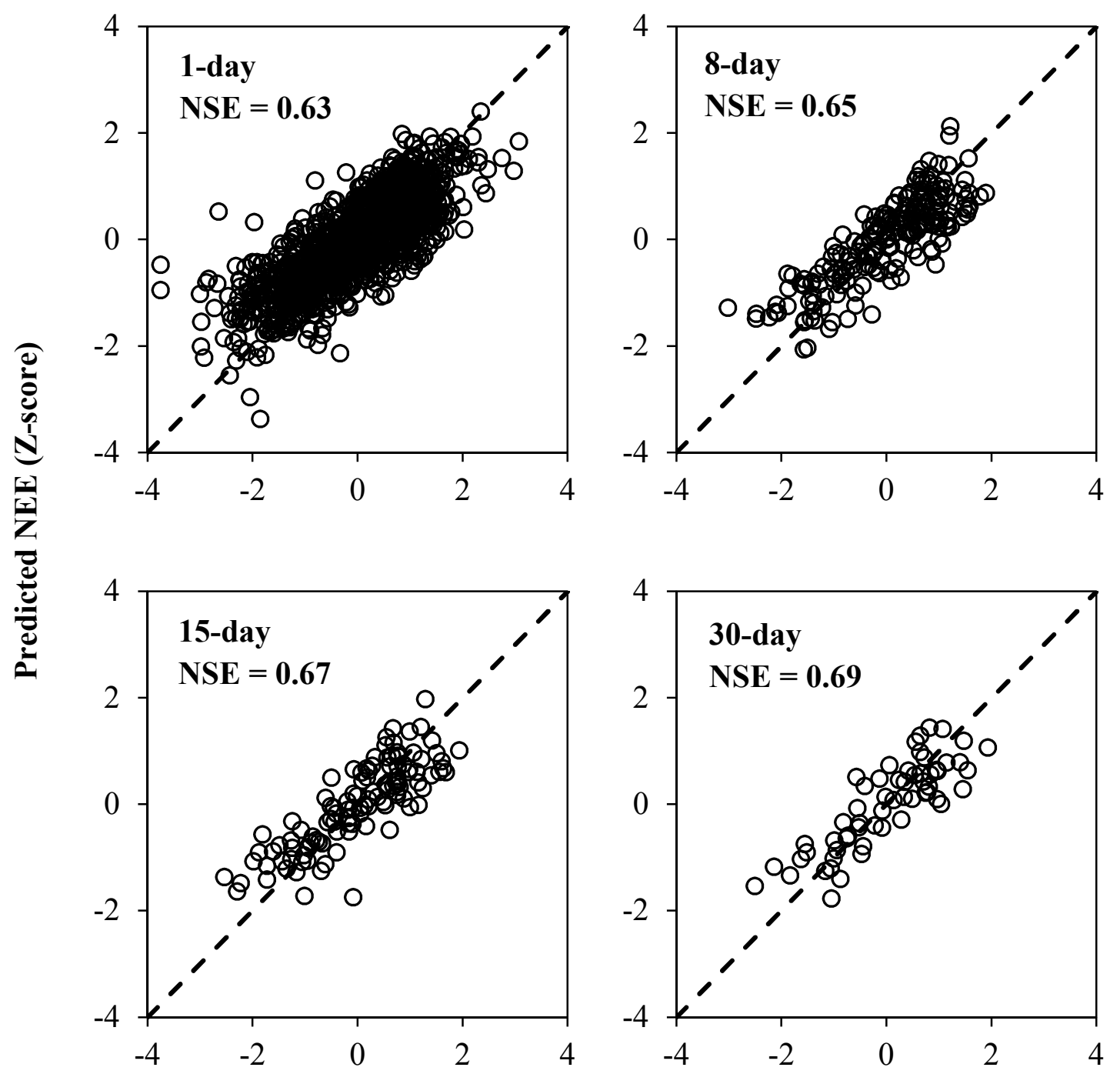

Observed NEE (Z-score)

Figure 5.2: Observed vs. predicted Z-scores of NEE at the different temporal scales. NEE was predicted using the Z-score PLSR model parameters $(\beta)$ obtained from 1000 MonteCarlo estimations. The dashed line represents the 1:1 line. 
Table 5.4: Z-score PLSR model parameters $(\beta)$ of NEE and standard deviations (in parenthesis) generated from 1000 Monte-Carlo estimations at the four temporal scales.

\begin{tabular}{lllll}
\hline Variables & 1-day & 8-day & 15-day & 30-day \\
\hline $\mathrm{Rn}$ & $-0.04(0.016)$ & $-0.07(0.041)$ & $-0.13(0.057)$ & $-0.16(0.081)$ \\
$\mathrm{PAR}$ & $-0.01(0.018)$ & $-0.03(0.031)$ & $-0.07(0.050)$ & $-0.09(0.066)$ \\
$\mathrm{LE}$ & $-0.37(0.019)$ & $-0.36(0.032)$ & $-0.34(0.044)$ & $-0.31(0.054)$ \\
$\mathrm{H}$ & $-0.55(0.030)$ & $-0.51(0.070)$ & $-0.52(0.083)$ & $-0.46(0.097)$ \\
$\mathrm{G}$ & $0.12(0.033)$ & $0.10(0.079)$ & $0.12(0.093)$ & $0.15(0.092)$ \\
$\mathrm{TA}$ & $-0.15(0.021)$ & $-0.11(0.053)$ & $-0.09(0.079)$ & $-0.08(0.092)$ \\
$\mathrm{TS}$ & $0.03(0.021)$ & $0.05(0.045)$ & $0.09(0.052)$ & $0.09(0.070)$ \\
$\mathrm{VPD}$ & $0.21(0.024)$ & $0.20(0.057)$ & $0.15(0.077)$ & $0.09(0.111)$ \\
$\mathrm{WL}$ & $0.18(0.031)$ & $0.18(0.078)$ & $0.15(0.096)$ & $0.14(0.088)$ \\
$\mathrm{SWC}$ & $-0.06(0.022)$ & $-0.05(0.044)$ & $-0.08(0.046)$ & $-0.10(0.049)$ \\
$\mathrm{WS}$ & $0.26(0.035)$ & $0.31(0.084)$ & $0.25(0.106)$ & $0.24(0.134)$ \\
$\mathrm{USTAR}$ & $-0.07(0.020)$ & $-0.15(0.048)$ & $-0.18(0.059)$ & $-0.24(0.065)$ \\
$\mathrm{CO} 2$ & $0.04(0.021)$ & $-0.07(0.045)$ & $-0.04(0.060)$ & $-0.07(0.078)$ \\
\hline Optimum PLS & 3 & 3 & 3 & 3 \\
$\mathrm{Components}$ & & 0.65 & 0.67 & 0.69 \\
\hline $\mathrm{NSE}$ & 0.63 & 0.59 & 0.57 & 0.55 \\
$\mathrm{RSR}$ & 0.61 & & & \\
\hline
\end{tabular}

Notes: NSE refers to the model fitting efficiency, whereas RSR refers to the accuracy of model predictions.

The standard deviations of the estimated $\beta$ obtained from the 1000 Monte-Carlo estimations indicated the uncertainty of the model parameters (Table 5.4). However, the standard deviations increased with the aggregation of time (i.e., for $\mathrm{Rn}$ standard deviation increased from 0.016 at 1-day to 0.081 at 30-day; Table 5.4), which indicated that the uncertainty of the model coefficients increased at higher temporal scales. For Rn, the linkages became weak to relatively moderate $(-0.04$ to -0.16$)$ whereas for PAR the linkages increased relatively weakly (-0.01 to 0.09). Among the flux variables, $\mathrm{H}$ showed the strongest linkages with NEE, although the linkages decreased (-0.55 to -0.46$)$ with increasing temporal scales. LE also showed similarly decreasing but less strong linkages $(-0.37$ to -0.31$)$ with NEE than $\mathrm{H}$. All of the variables of the 'radiationenergy (RE)' component exerted dominance towards $\mathrm{CO}_{2}$ uptake except for $\mathrm{G}$, which moderately (0.10 to 0.15$)$ dominated $\mathrm{CO}_{2}$ release and remained relatively stable over the different temporal scales. The variables from the 'temperature-hydrology (TH)' component depicted weak to 
moderate linkages towards NEE with mixed dominance over both $\mathrm{CO}_{2}$ uptake and release. Both TA and SWC indicated influence on $\mathrm{CO}_{2}$ uptake, where linkages of TA decreased (-0.15 to -0.08$)$ and SWC increased (-0.06 to -0.10$)$ along the aggregated temporal scales. On the contrary, VPD and WL decreased ( 0.21 to 0.09 and 0.18 to 0.14 respectively) at higher temporal scales exerting control over $\mathrm{CO}_{2}$ release.

TS also showed weak control over $\mathrm{CO}_{2}$ release, where the linkages increased (0.03 to 0.09$)$ from 1-day to 30-day scale. In the 'aerodynamic (AD)' component, USTAR dominated its influence over $\mathrm{CO}_{2}$ uptake, where the linkages increased from weak to moderate ( -0.07 to -0.24$)$ at higher temporal scales. WS showed moderate linkages (0.26) at 1-day scale, which slightly increased (0.31) at 8-day scale and then started decreasing $(0.31$ to 0.24$)$ at the higher scales of temporal aggregation, influencing $\mathrm{CO}_{2}$ release. The ambient $\mathrm{CO}_{2}$ concentration exhibited weak linkages with NEE, regulating $\mathrm{CO}_{2}$ release (0.04) at 1-day scale and shifting its influence on $\mathrm{CO}_{2}$ uptake (-0.04 to -0.07$)$ at higher temporal scales.

The $95 \%$ confidence limits of the estimated $\beta$ from 1000 Monte-Carlo estimations were used to determine the $95 \%$ confidence limits of the aggregated linkages that clearly showed increasing uncertainty towards higher temporal scales (Figure 5.3). 


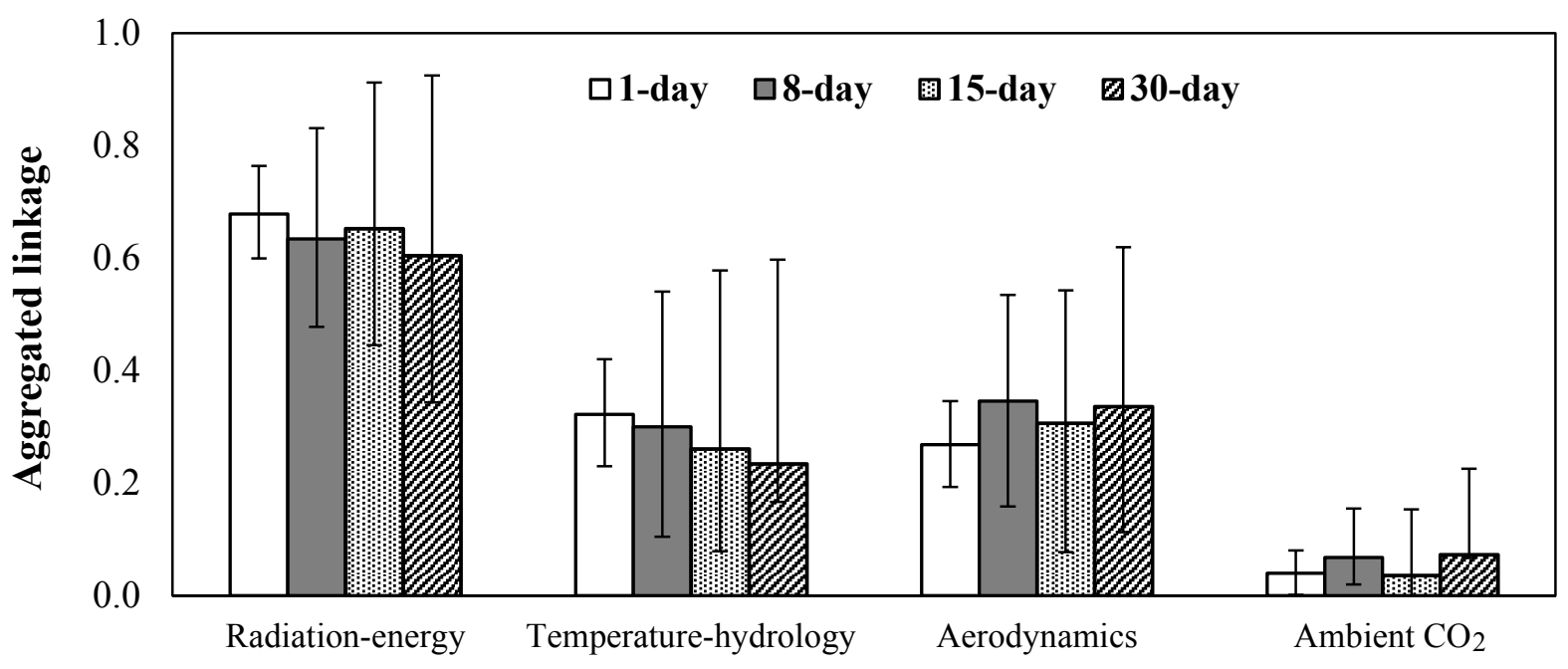

Figure 5.3: Aggregated linkages of NEE with the major biophysical process components at the different temporal scales. Error bars represent $95 \%$ confidence limits of the aggregated linkages.

In general, the RE component represented the strongest linkages with NEE followed by the moderate linkages of the $\mathrm{TH}$ and $\mathrm{AD}$ components. The $\mathrm{AC}$ component showed the weakest linkages with NEE. The linkages of both RE and TH components decreased at higher temporal scales whereas the linkages of $\mathrm{AD}$ and $\mathrm{AC}$ slightly increased from 1-day to 8-day scales and then remained stable at the remaining scales of temporal aggregation (Figure 5.3). 


\section{CHAPTER 6: DISCUSSION}

\subsection{On the hourly linkages across diverse ecosystems}

The climatic and ecohydrologic linkages - computed from the three-step data analytics methodology — were comparable across the six diverse ecosystems. The results were consistent with the study conducted by Ishtiaq and Abdul-Aziz (2015) that reported similar grouping patterns and linkages of climatic and ecohydrological drivers of $\mathrm{CO}_{2}$ fluxes across eight deciduous forests. The 'radiation-energy (RE)' component exhibited the strongest linkages with NEE across all the ecosystem types, which is in accordance with the earlier studies (Schmidt et al., 2011, Jung et al., 2011, Fang and Michalak, 2015). Among all the variables, LE showed the strongest linkages with NEE across all the ecosystems, although similar linkages of $\mathrm{H}$ and LE in WET depicted a strong coupling among the carbon, energy, and water fluxes for that ecosystem. Mechanistically, VPD regulates stomatal closure of plants, which in concert represents the decrease of ecosystem $\mathrm{CO}_{2}$ uptake (Heber et al., 1986). This is caused by the lowering of LE to preserve water for resuming plant's metabolic activities. WET showed higher availability of water (see table 3.2; SWC $\approx 79 \%$ ) compared to the other ecosystems (see table $3.2 ; \mathrm{SWC} \approx 17$ to $31 \%$ ). Due to this high water availability in WET, the sensitivity of VPD to stomatal closure decreases causing the relatively lower linkages of LE with NEE compared to the higher linkages of LE in all the other ecosystems. Lack of energy balance closure is another important issue that could affect the estimated linkages. The linkages of the energy and heat flux variables, particularly G might be affected by this issue for WET (Mackay et al., 2007, Schedlbauer et al, 2011, Hossen et al., 2012). According to recent studies conducted in WET, the lacking energy balance $(\sim 70-80 \%)$ is typically closed by means of an extra energy storage term found within the water column. Although the energy balance closure 
attempted by this approach might not always be effective as the closing of the energy balance is entirely dependent on including the fluxes at the greater heterogenous landscape (Foken, 2008).

The 'temperature-hydrology (TH)' component was identified as the second most dominant biophysical process component exhibiting moderate linkages with NEE. The signs of $\beta$ for the temperature variables indicated influence on ecosystem respiration (Mahecha et al., 2010, YvonDurocher et al., 2012). The variations of temperature and soil water availability could have a substantial effect on the changes in energy and water fluxes of plants (Loescher et al., 2003). The influence of soil temperature on soil respiration, in particular, could be defined by the classical Arrhenius-type exponential model (Lloyd and Taylor, 1994). The linkages of SWC were NEE was weak across the diverse ecosystems, which could be attributed by the low hourly variations of SWC. A study conducted by Albert et al. (2017) presented for ENF reported an entangled relationship between the effects of temperature and hydrological variables on NEE. This supports the formation of the 'temperature-hydrology' group combining temperature variables along with SWC. Weak correlation was observed between soil water and photosynthesis at short temporal scales by Desai (2014).

Previous studies supported the findings of the weak hourly linkages of NEE with the 'aerodynamic (AD)' component (Schmidt et al., 2011). Using the half-hourly data in a tropical wet forest in Central America, Loescher et al. (2003) found weak relationship between USTAR and NEE. A significant finding of this study was the relatively stronger linkages of the AD component for the low canopy ecosystems (i.e., CRO, GRA and WET). Such linkages represented the greater effect of shear-driven processes initiated by wind on NEE. The smaller canopy height along with the eddy covariance sensors resulted in a stronger coupling of NEE with turbulence. 
The data analytics indicated moderate linkages of the 'ambient atmospheric $\mathrm{CO}_{2}(\mathrm{AC})$ ' exhibiting its influence on ecosystem respiration as the signs of $\beta$ were consistently positive across the ecosystems. The $\mathrm{CO}_{2}$ fertilization effect, which is reflected over decades was not effective in the quantified linkages due to the short term records of the datasets.

\subsection{On the multi-temporal linkages at the Everglades short hydro-period site}

The strong linkages of the 'radiation-energy (RE)' component indicated radiation, energy, and heat flux variables as the primary drivers of NEE at the Everglades short hydro-period site for the different temporal scales (1-day, 8-day, 15-day, and 30-day). Both $\mathrm{H}$ and LE exhibited superior linkages with NEE across the temporal scales; however, the linkages of $\mathrm{H}$ were relatively higher than LE linkages. In the Everglades, the sources of latent heat are less compared to the sources of sensible heat due to the presence of standing water and aquatic vegetation (German, 2000). This might be the reason why linkages of LE were relatively smaller than H. LE indicates an important link with NEE, as photosynthesis releases water vapor while storing carbon (indicating $\mathrm{CO}_{2}$ uptake) (Shoemaker et al. 2015). H on the other hand, impacts ET by influencing the transport of water vapor towards the atmosphere. The strong linkages of $\mathrm{H}$ and LE indicated a coupling among the carbon, energy, and water fluxes, which was also observed in the hourly linkages for WET ecosystems. However, the linkages of both H and LE decreased with increasing temporal scales, indicating a potential decoupling of the fluxes at higher temporal scales. The decoupling of NEE and ET at higher temporal scales (e.g., weekly) could be linked with the warm and humid summer climate of the study site (Wagle et al. 2016). As ET decoupled from NEE at higher temporal scales, the linkages of LE and H decreased from 1-day to 30-day scale. The signs of $\beta$ for Rn and PAR exhibited influence on the $\mathrm{CO}_{2}$ uptake signal of NEE. Solar radiation drives photosynthesis that contributes to the uptake of $\mathrm{CO}_{2}$ by plants (Jimenez et al., 2012, Zhong et al. 2016). G depends on 
the temperature difference between the surface and the underlying soil (Bonan, 2015). In the analysis, $\mathrm{G}$ exhibited dominance on ecosystem respiration, which is understandable as the temperature gradient might induce soil respiration. The linkages of $G$ with NEE remained relatively stable through 1-day to 15 -day scale but increased at the 30 -day scale. The linkage patterns indicated a weak variation of $G$ up to the 15-day scale where the variation got relatively stronger after that.

The linkages of the 'temperature-hydrology $(\mathrm{TH})$ ' component at the different temporal scales characterized the influence of the temperature and hydrologic variables as the second most important drivers of NEE at the Everglades short hydro-period site. The temperature variables control both photosynthesis and respiration (Ryan and Law, 2005). TA yields favorable environmental conditions that allow photosynthesis to occur (Bonan, 2015) whereas TS regulates soil respiration by stimulating microbial reactions in the soil (Karhu et al., 2014). The linkages of both TA and TS with NEE found in the analysis were therefore consistent with the current literature. The variability of NEE is influenced by the biological responses to climatic variability at longer temporal scales (Dietze et al., 2011). The increasing linkages of TS towards stronger respiration from 1-day to 30-day scale complied with this mechanism. Therefore, at higher temporal scales biological processes are strongly influenced by TS resulting in greater respiration. Overall, the linkages of hydrologic variables represented their decreasing influence on respiration with increasing temporal scales. The aerobic respiration of soil is hindered as water fills up the pores in the soil. The additional water in the soil renders direct impediment to gas diffusion that suppresses soil respiration (Guo et al. 2009). In addition, the linkages of SWC with NEE increased slightly with the higher temporal scales that might be because uptake of water by plants from the soil in the Everglades short hydro-period site follows a seasonal pattern (Wei et al. 2013). 
Increasing VPD results in less uptake of $\mathrm{CO}_{2}$ as increased atmospheric moisture demand accelerates stomatal closure (Loescher et al. 2003, Jimenez et al., 2012). Similar to the RE component, linkages of the TH component also decreased with increasing temporal scales.

The aerodynamic variables act as transport mechanisms for the $\mathrm{CO}_{2}$ exchanges between the ecosystem and the atmosphere. Vegetation absorbs $\mathrm{CO}_{2}$ during photosynthesis whereas wind mixes the air and restores the absorbed $\mathrm{CO}_{2}$ in the atmosphere (Bonan, 2015). The aerodynamic variables showed moderate linkages with NEE for the different temporal scales. In the case of aquatic ecosystems, wind plays an important role in the $\mathrm{CO}_{2}$ exchanges across the air-water interface (Cole and Caraco, 1998). Overall, the moderate linkages of the 'aerodynamic (AD)' were similar to that of the TH component. The comparatively stronger linkages of the AD component might be because of the low canopy nature of the Everglades short hydro-period site. The low canopy nature could induce greater shear driven effects. The relatively invariable nature of the AD component across temporal scales (8-day to 30-day) indicated the weak influence of seasonality.

The linkages of the 'ambient atmospheric $\mathrm{CO}_{2}$ (AC)' component with NEE at 1-day temporal scale exerted weak control over respiratory fluxes whereas at higher temporal scales (8day to 30-day) it showed weak dominance on photosynthetic fixation. At the higher temporal scales, the $\mathrm{CO}_{2}$ fertilization effect might kick in that could be the reason why the sign of $\beta$ for the ambient concentration of $\mathrm{CO}_{2}$ changed at higher temporal scales. The linkages were weak across the temporal scales that might be because of almost equal linkages of atmospheric $\mathrm{CO}_{2}$ concentration influencing $\mathrm{CO}_{2}$ uptake and respiration signal of NEE (Malone et al., 2015).

\subsection{Implications of estimated linkages}

The study determined the climatic and ecohydrologic linkages of NEE estimated from optimal Zscore PLSR models. These linkages represented the global sensitivities of NEE towards its drivers 
across spatiotemporal scales (Haan, 2002; Saltelli et al., 2008). The study reiterated the importance of the 'radiation-energy' component in modeling terrestrial $\mathrm{CO}_{2}$ fluxes. Therefore, the radiation and energy flux variables are required to be better defined when developing mechanistic terrestrialbiosphere models (Fang and Michalak, 2015). The study showed comparable linkages of the 'radiation-energy' and 'temperature-hydrology' components across spatiotemporal scales and further identified the most important drivers of NEE across space and time. Therefore, the findings of this study will help in the development of spatiotemporally robust low-dimensional models of NEE. Furthermore, empirical models can be developed with these important drivers (PAR, LE, TA, VPD, and WS) using non-standardized data, which can be utilized to diagnose and investigate the reliability and accuracy of mechanistic models of NEE (Beer et al., 2010; Keenan et al., 2012).

The robustness of the estimated climatic and ecohydrologic linkages of NEE is an indication of an equilibrium state in the ecosystem metabolic activities. The equilibrium state fundamentally refers that under the gradually changing climate conditions, the biophysical process components would adapt through the ecological resilience mechanisms at an ecosystem (Scheffer et al., 2001). Although, in the case of rapidly changing climate conditions, the optimum adaptation capacity of an ecosystem might be surpassed and impede the equilibrium state of ecosystem metabolism. Therefore, future studies should conduct experiments regarding the stability of the climatic and ecohydrologic linkages due to shift of ecosystem metabolic equilibrium caused by the rapidly changing climatic conditions.

An important finding of the study is the highest linkage of LE with NEE across all the ecosystems. The greater linkages of LE with NEE refer to the negative impact on ecosystem metabolism due to the changing climate. A continental-scale drying of the atmosphere with higher VPD is expected to increase the atmospheric demand, resulting in greater stomatal closure of the 
plants (Novick et al., 2016, Ficklin and Novick, 2017). The effect of greater linkages of LE with NEE coupled with the moderate linkages of the temperature and hydrological variables refered to similar negative impacts on ecosystem metabolism because of heat waves and draughts (van der Molen et al., 2011, Zhang et al., 2016). Such negative impact on ecosystem metabolism will reduce the $\mathrm{CO}_{2}$ uptake by the plants and therefore diminish the potential for sequestering carbon by the terrestrial ecosystems. 


\section{CHAPTER 7: CONCLUSIONS}

The climatic and ecohydrologic linkages of hourly NEE were investigated by determining their linkages utilizing a systematic data analytics approach for six diverse ecosystems (evergreen

forest, deciduous forest, mixed forest, cropland, grassland, and wetland) comprising of 57 eddy covariance flux tower sites under the AmeriFlux network. This study further investigated the climatic and ecohydrologic linkages of NEE across four temporal scales (1-day, 8-day, 15-day, and 30-day) for a case study site at Florida Everglades short hydro-period marsh. The analytics identified four major biophysical process components ('radiation-energy', 'temperaturehydrology', 'aerodynamic', and 'atmospheric $\mathrm{CO}_{2}$ ') that mainly drive NEE. The linkages of these components were comparable across the spatiotemporal scales.

The important findings of this study can be highlighted as follows:

- The 'radiation-energy' component exhibited the highest linkages with NEE indicating the strongest dominance of solar radiation and energy fluxes on ecosystem $\mathrm{CO}_{2}$ fluxes.

- The 'temperature-hydrology' component showed moderate linkages with NEE illustrating that the temperature and hydrologic variables are the second most important drivers of terrestrial $\mathrm{CO}_{2}$ fluxes.

- The dominating linkage patterns of both the 'radiation-energy' and 'temperaturehydrology' components were consistent across the different temporal scales for the Florida short hydroperiod marsh site (1-day, 8-day, 15-day, and 30-day).

- Latent heat fluxes (LE) exhibited the strongest linkages with NEE among all the variables across spatiotemporal scales except in the case of wetlands where sensible heat flux $(H)$ and LE showed similar linkages at the 1-hour scale. 
- The 'aerodynamic' component in general exhibited weak linkages with NEE. However, relatively stronger linkages of this component were observed for the smaller canopy ecosystems (i.e., cropland, grassland and wetland) illustrating the shear-driven effect of aerodynamic variables on NEE compared to ecosystems with higher canopy.

- The 'atmospheric $\mathrm{CO}_{2}$ ' component showed moderate linkages with NEE across different ecosystems in the 1-hour scale. However, the linkages decreased with temporal scale for the Florida Everglades case study sites.

The linkages provided valuable insights into the biophysical process variables of ecosystem $\mathrm{CO}_{2}$ fluxes across spatial and temporal scales. The findings would help to develop spatiotemporally robust, low-dimensional models to reliably predict NEE under a variable climate and environment. 


\section{REFERENCES}

Abdul-Aziz, O. I., and Ahmed S. (2017). Relative linkages of stream water quality and environmental health with the land use and hydrologic drivers in the coastal-urban watersheds of southeast Florida. GeoHealth, 1, 180-195, doi:10.1002/2017GH000058.

Abdul-Aziz, O. I., and Ishtiaq K. S. (2014). Robust empirical modelling of dissolved oxygen in small rivers and streams: scaling by a single reference observation. Journal of Hydrology, 511, 648-657, doi:10.1016/j.jhydrol.2014.02.022.

Akaike, H. (1974). A new look at the statistical model identification. IEEE Transactions on Automatic Control, 19(6), 716-723.

Albert, L. P., Keenan, T. F., Burns, S. P., Huxman, T. E., and Monson, R. K. (2017). Climate controls over ecosystem metabolism: insights from a fifteen-year inductive artificial neural network synthesis for a subalpine forest. Oecologia, 184(1), 25-41.

AmeriFlux. (2016). AmeriFlux site and data exploration system. Retrieved from http://ameriflux.lbl.gov/data/download-data/ (Accessed on September 29, 2016)

Amiro, B. D., Barr, A. G., Black, T. A., Iwashita, H., Kljun, N., McCaughey, J. H., ... and Saigusa, N. (2006). Carbon, energy and water fluxes at mature and disturbed forest sites, Saskatchewan, Canada. Agricultural and Forest Meteorology, 136(3), 237-251, doi: 10.1016/j.agrformet.2004.11.012.

Arain, M. A., and Restrepo-Coupe, N. (2005). Net ecosystem production in a temperate pine plantation in southeastern Canada. Agricultural and Forest Meteorology, 128(3), 223241, doi:10.1016/j.agrformet.2004.10.003. 
Aubinet, M., Vesala, T., and Papale, D. (Eds.). (2012). Eddy Covariance: A Practical Guide to Measurement and Data Analysis. London: Springer Science and Business Media.

Baldocchi, D., Chu, H., and Reichstein, M. (2017). Inter-annual variability of net and gross ecosystem carbon fluxes: A review. Agricultural and Forest Meteorology. doi: 10.1016/j.agrformet.2017.05.015.

Baldocchi, D., Falge, E., Gu, L., Olson, R., Hollinger, D., Running, S., ... and Fuentes, J. (2001). FLUXNET: A new tool to study the temporal and spatial variability of ecosystem-scale carbon dioxide, water vapor, and energy flux densities. Bulletin of the American Meteorological Society, 82(11), 2415-2434.

Barr, J. G., Engel, V., Fuentes, J. D., Zieman, J. C., O'Halloran, T. L., Smith, T. J., and Anderson, G. H. (2010). Controls on mangrove forest-atmosphere carbon dioxide exchanges in western Everglades National Park. Journal of Geophysical Research: Biogeosciences, 115, G02020. doi: 10.1029/2009JG001186.

Beer, C., Reichstein, M., Tomelleri, E., Ciais, P., Jung, M., Carvalhais, N., ... and Bondeau, A. (2010). Terrestrial gross carbon dioxide uptake: global distribution and covariation with climate. Science, 329(5993), 834-838.

Bergeron, O., Margolis, H. A., Black, T. A., Coursolle, C., Dunn, A. L., Barr, A. G., and Wofsy, S. C. (2007). Comparison of carbon dioxide fluxes over three boreal black spruce forests in Canada. Global Change Biology, 13(1), 89-107.

Bonan, G. (2015). Ecological Climatology: Concepts and Applications. New York, NY: Cambridge University Press. 
Chu, H., Chen, J., Gottgens, J. F., Ouyang, Z., John, R., Czajkowski, K., and Becker, R. (2014). Net ecosystem methane and carbon dioxide exchanges in a Lake Erie coastal marsh and a nearby cropland. Journal of Geophysical Research: Biogeosciences, 119(5), 722-740, doi: $10.1002 / 2013 J G 002520$.

Chu, H., Gottgens, J. F., Chen, J., Sun, G., Desai, A. R., Ouyang, Z., ... and Czajkowski, K. (2015). Climatic variability, hydrologic anomaly, and methane emission can turn productive freshwater marshes into net carbon sources. Global Change Biology, 21(3), 1165-1181, doi: $10.1111 / \mathrm{gcb} .12760$.

Clark, K. L., Skowronski, N. S., Gallagher, M. R., Renninger, H., and Schäfer, K. V. R. (2014). Contrasting effects of invasive insects and fire on ecosystem water use efficiency. Biogeosciences, 11(23), 6509-6523.

Cole, J. J., and Caraco, N. F. (1998). Atmospheric exchange of carbon dioxide in a low-wind oligotrophic lake measured by the addition of $\mathrm{SF}_{6}$. Limnology and Oceanography, 43(4), 647-656.

Coursolle, C., Giasson, M. A., Margolis, H. A., and Bernier, P. Y. (2012). Moving towards carbon neutrality: $\mathrm{CO}_{2}$ exchange of a black spruce forest ecosystem during the first 10 years of recovery after harvest. Canadian Journal of Forest Research, 42(11), 19081918.

Danner, B. T., and Knapp, A. K. (2003). Abiotic constraints on the establishment of Quercus seedlings in grassland. Global Change Biology, 9(2), 266-275. 
Davidson, E. A., Richardson, A. D., Savage, K. E., and Hollinger, D. Y. (2006). A distinct seasonal pattern of the ratio of soil respiration to total ecosystem respiration in a sprucedominated forest. Global Change Biology, 12(2), 230-239.

De Jong, S. (1993). SIMPLS: an alternative approach to partial least squares regression. Chemometrics and Intelligent Laboratory Systems, 18(3), 251-263.

Desai, A. R. (2010). Climatic and phenological controls on coherent regional interannual variability of carbon dioxide flux in a heterogeneous landscape. Journal of Geophysical Research: Biogeosciences, 115, 1-13, doi: 10.1029/2010JG001423.

Desai, A. R. (2014), Influence and predictive capacity of climate anomalies on daily to decadal extremes in canopy photosynthesis. Photosynthesis Research, 119(1-2), 31-47, doi: 10.1007/s11120-013-9925-Z.

Desai, A. R., Moore, D. J., Ahue, W. K., Wilkes, P. T., De Wekker, S. F., Brooks, B. G., ... and Quaife, T. (2011). Seasonal pattern of regional carbon balance in the central Rocky Mountains from surface and airborne measurements. Journal of Geophysical Research: Biogeosciences, 116, G04009, doi: 10.1029/2011JG001655.

Dietze, M. C., Vargas, R., Richardson, A. D., Stoy, P. C., Barr, A. G., Anderson, R. S., ... and Ciais, P. (2011). Characterizing the performance of ecosystem models across time scales: A spectral analysis of the North American Carbon Program site-level synthesis. Journal of Geophysical Research: Biogeosciences, 116, G04029, doi: 10.1029/2011JG001661.

Dore, S., Montes-Helu, M., Hart, S. C., Hungate, B. A., Koch, G. W., Moon, J. B., ... and Kolb, T. E. (2012). Recovery of ponderosa pine ecosystem carbon and water fluxes from thinning and stand-replacing fire. Global Change Biology, 18(10), 3171-3185. 
Dragon, K. (2006). Application of factor analysis to study contamination of a semi-confined aquifer (Wielkopolska Buried Valley aquifer, Poland). Journal of Hydrology, 331(1), 272-279, doi: 10.1016/j.jhydrol.2006.05.032.

Dragoni, D., Schmid, H. P., Wayson, C. A., Potter, H., Grimmond, C. S. B., and Randolph, J. C. (2011). Evidence of increased net ecosystem productivity associated with a longer vegetated season in a deciduous forest in south-central Indiana, USA. Global Change Biology, 17(2), 886-897.

EDEN. (2016). Everglades Depth Estimation Network (EDEN) for Support of Biological and Ecological Assessments. Retrieved from https://sofia.usgs.gov/eden/ (Accessed on September 29, 2016)

Fang, Y., and Michalak, A. M. (2015). Atmospheric observations inform $\mathrm{CO}_{2}$ flux responses to enviroclimatic drivers. Global Biogeochemical Cycles, 29(5), 555-566, doi:10.1002/2014GB005034.

Ficklin, D., and K. Novick (2017). Historic and projected changes in evaporative demand suggest a continental-scale drying of the U.S. atmosphere, Journal of Geophysical Research: Atmospheres, 122(4), 2061-2079, doi: 10.1002/2016JD025855.

Foken, T. (2008). The energy balance closure problem: an overview. Ecological Applications, 18(6), 1351-1367, doi:10.1890/06-0922.1.

Frank, J. M., Massman, W. J., Ewers, B. E., Huckaby, L. S., and Negrón, J. F. (2014). Ecosystem $\mathrm{CO}_{2} / \mathrm{H}_{2} \mathrm{O}$ fluxes are explained by hydraulically limited gas exchange during tree mortality from spruce bark beetles. Journal of Geophysical Research: Biogeosciences, 119(6), 1195-1215, doi:10.1002/2013JG002597. 
German, E.R. (2000) Regional Evaluation of Evapotranspiration in the Everglades: USGS Water-Resources Investigations Report (00-4217). Washington, DC: US Geological Survey.

Gough, C. M., Hardiman, B. S., Nave, L. E., Bohrer, G., Maurer, K. D., Vogel, C. S., ... and Curtis, P. S. (2013). Sustained carbon uptake and storage following moderate disturbance in a Great Lakes forest. Ecological Applications, 23(5), 1202-1215.

Gu, L., Meyers, T., Pallardy, S. G., Hanson, P. J., Yang, B., Heuer, M., ... and Wullschleger, S. D. (2006). Direct and indirect effects of atmospheric conditions and soil moisture on surface energy partitioning revealed by a prolonged drought at a temperate forest site. Journal of Geophysical Research: Atmospheres, 111, D16102, doi:10.1029/2006JD007161.

Guo, H., Noormets, A., Zhao, B., Chen, J., Sun, G., Gu, Y., ... and Chen, J. (2009). Tidal effects on net ecosystem exchange of carbon in an estuarine wetland. Agricultural and Forest Meteorology, 149(11), 1820-1828.

Haan, C. T. (2002). Statistical Methods in Hydrology. Ames, IA: The Iowa State University Press.

Hatala, J. A., Detto, M., Sonnentag, O., Deverel, S. J., Verfaillie, J., and Baldocchi, D. D. (2012). Greenhouse gas $\left(\mathrm{CO}_{2}, \mathrm{CH}_{4}, \mathrm{H}_{2} \mathrm{O}\right)$ fluxes from drained and flooded agricultural peatlands in the Sacramento-San Joaquin Delta. Agriculture, Ecosystems and Environment, 150, 118.

Heber, U., Neimanis, S., and Lange, O. L. (1986). Stomatal aperture, photosythesis and water fluxes in mesophyll cells as affected by the abscission of leaves. Simultaneous 
measurements of gas exchange, light scattering and chlorphyll fluorescence. Planta, 167(4), 554-562.

Heskel, M. A., Atkin, O. K., Turnbull, M. H., and Griffin, K. L. (2013). Bringing the Kok effect to light: a review on the integration of daytime respiration and net ecosystem exchange. Ecosphere, 4(8), 1-14.

Hiller, R. V., McFadden, J. P., and Kljun, N. (2011). Interpreting $\mathrm{CO}_{2}$ fluxes over a suburban lawn: the influence of traffic emissions. Boundary Layer Meteorology, 138(2), 215-230, doi:10.1007/s10546-010-9558-0.

Hossen, M., Mano, M., Miyata, A., Baten, M. and Hiyama, T. (2012). Surface energy partitioning and evapotranspiration over a double-cropping paddy field in Bangladesh. Hydrological Processes, 26(9), 1311-1320.

Huxman, T.E., Turnipseed, A.A., Sparks, J.P., Harley, P.C. and Monson, R.K. (2003). Temperature as a control over ecosystem $\mathrm{CO}_{2}$ fluxes in a high-elevation, subalpine forest. Oecologia, 134(4), 537-546.

Ishtiaq, K. S., and Abdul-Aziz, O. I. (2015). Relative Linkages of Canopy-Level $\mathrm{CO}_{2}$ Fluxes with the Climatic and Environmental Variables for US Deciduous Forests. Environmental Management, 55(4), 943-960.

Irvine, J., Law, B. E., Kurpius, M. R., Anthoni, P. M., Moore, D., and Schwarz, P. A. (2004). Age-related changes in ecosystem structure and function and effects on water and carbon exchange in ponderosa pine. Tree Physiology, 24(7), 753-763. 
Janssen, H. (2013). Monte-Carlo based uncertainty analysis: Sampling efficiency and sampling convergence. Reliability Engineering and System Safety, 109, 123-132.

Jimenez, K. L., Starr, G., Staudhammer, C. L., Schedlbauer, J. L., Loescher, H. W., Malone, S. L., and Oberbauer, S. F. (2012). Carbon dioxide exchange rates from short-and longhydroperiod Everglades freshwater marsh. Journal of Geophysical Research: Biogeosciences, 117, G04009, doi:10.1029/2012JG002117.

Jolliffe, I. T. (2002). Principal Component Analysis. New York, NY: Springer-Verlag.

Jung, M., Reichstein, M., Margolis, H. A., Cescatti, A., Richardson, A. D., Arain, M. A., ... and Gianelle, D. (2011). Global patterns of land-atmosphere fluxes of carbon dioxide, latent heat, and sensible heat derived from eddy covariance, satellite, and meteorological observations. Journal of Geophysical Research: Biogeosciences, 116, G00J07. doi: 10.1029/2010jg001566.

Karhu, K., Auffret, M. D., Dungait, J. A., Hopkins, D. W., Prosser, J. I., Singh, B. K., ... and Gouriveau, F. (2014). Temperature sensitivity of soil respiration rates enhanced by microbial community response. Nature, 513(7516), 81-84, doi:10.1038/nature13604.

Keenan, T. F., Davidson, E., Moffat, A. M., Munger, W., and Richardson, A. D. (2012). Using model-data fusion to interpret past trends, and quantify uncertainties in future projections, of terrestrial ecosystem carbon cycling. Global Change Biology, 18(8), 2555-2569.

Kim, S. (2013). Ancillary Data Report: Landcover Classification. Pasadena, CA: Jet Propulsion Laboratory, California Institute of Technology. 
Knox, S. H., Matthes, J. H., Sturtevant, C., Oikawa, P. Y., Verfaillie, J., and Baldocchi, D. (2016). Biophysical controls on interannual variability in ecosystem-scale $\mathrm{CO}_{2}$ and $\mathrm{CH}_{4}$ exchange in a California rice paddy. Journal of Geophysical Research: Biogeosciences, 121(3), 978-1001.

Knox, S. H., Sturtevant, C., Matthes, J. H., Koteen, L., Verfaillie, J., and Baldocchi, D. (2015). Agricultural peatland restoration: effects of land-use change on greenhouse gas $\left(\mathrm{CO}_{2}\right.$ and $\mathrm{CH}_{4}$ ) fluxes in the Sacramento-San Joaquin Delta. Global Change Biology, 21(2), 750765.

Kolb, T., Dore, S., and Montes-Helu, M. (2013). Extreme late-summer drought causes neutral annual carbon balance in southwestern ponderosa pine forests and grasslands. Environmental Research Letters, 8(1), doi:10.1088/1748-9326/8/1/015015.

Kuhn, M., and Johnson, K. (2013). Applied Predictive Modeling (Vol. 810). New York, NY: Springer.

Law, B. E., Falge, E., Gu, L. V., Baldocchi, D. D., Bakwin, P., Berbigier, P., ... and Goldstein, A. (2002). Environmental controls over carbon dioxide and water vapor exchange of terrestrial vegetation. Agricultural and Forest Meteorology, 113(1), 97-120.

Lloyd, J., and Taylor, J. A. (1994). On the temperature dependence of soil respiration. Functional Ecology, 8, 315-323.

Loescher, H. W., Oberbauer, S. F., Gholz, H. L., and Clark, D. B. (2003). Environmental controls on net ecosystem-level carbon exchange and productivity in a Central American tropical wet forest. Global Change Biology, 9(3), 396-412. 
Mackay, D. S., Ewers, B. E., Cook, B. D., and Davis, K. J. (2007). Environmental drivers of evapotranspiration in a shrub wetland and an upland forest in northern Wisconsin. Water Resources Research, 43(3), W03442, doi:10.1029/2006WR005149.

Mahbub, P., Ayoko, G. A., Goonetilleke, A., Egodawatta, P., and Kokot, S. (2010). Impacts of traffic and rainfall characteristics on heavy metals build-up and wash-off from urban roads. Environmental Science and Technology, 44(23), 8904-8910.

Mahecha, M. D., Reichstein, M., Carvalhais, N., Lasslop, G., Lange, H., Seneviratne, S. I., ... and Janssens, I. A. (2010). Global convergence in the temperature sensitivity of respiration at ecosystem level. Science, 329(5993), 838-840, doi:10.1126/science.1189587.

Malone, S. L., Keough, C., Staudhammer, C. L., Ryan, M. G., Parton, W. J., Olivas, P., ... and Starr, G. (2015). Ecosystem resistance in the face of climate change: a case study from the freshwater marshes of the Florida Everglades. Ecosphere, 6(4), 1-23, doi: 10.1890/ES14-00404.1.

Matthes, J. H., Knox, S. H., Sturtevant, C., Sonnentag, O., Verfaillie, J., and Baldocchi, D. (2015). Predicting landscape-scale $\mathrm{CO}_{2}$ flux at a pasture and rice paddy with long-term hyperspectral canopy reflectance measurements. Biogeosciences, 12(15), 4577-4594.

Matamala, R., Jastrow, J. D., Miller, R. M., and Garten, C. T. (2008). Temporal changes in C and $\mathrm{N}$ stocks of restored prairie: implications for $\mathrm{C}$ sequestration strategies. Ecological Applications, 18(6), 1470-1488.

McCaughey, J. H., Pejam, M. R., Arain, M. A., and Cameron, D. A. (2006). Carbon dioxide and energy fluxes from a boreal mixedwood forest ecosystem in Ontario, Canada. 
Agricultural and Forest Meteorology, 140(1), 79-96, doi:10.1016/j.agrformet.2006.08.010.

Miao, S. L., and Sklar, F. H. (1997). Biomass and nutrient allocation of sawgrass and cattail along a nutrient gradient in the Florida Everglades. Wetlands Ecology and Management, $5(4), 245-263$.

Miller, R. L., and Fujii, R. (2010). Plant community, primary productivity, and environmental conditions following wetland re-establishment in the Sacramento-San Joaquin Delta, California. Wetlands Ecology and Management, 18(1), 1-16.

Mitsch, W. J., Bernal, B., Nahlik, A. M., Mander, Ü., Zhang, L., Anderson, C. J., ... and Brix, H. (2013). Wetlands, carbon, and climate change. Landscape Ecology, 28(4), 583-597.

Moriasi, D. N., Arnold, J. G., Van Liew, M. W., Bingner, R. L., Harmel, R. D., and Veith, T. L. (2007). Model evaluation guidelines for systematic quantification of accuracy in watershed simulations. Transactions of the ASABE, 50(3), 885-900.

Morin, T. H., Bohrer, G., Stefanik, K. C., Rey-Sanchez, A. C., Matheny, A. M., and Mitsch, W. J. (2017). Combining eddy-covariance and chamber measurements to determine the methane budget from a small, heterogeneous urban floodplain wetland park. Agricultural and Forest Meteorology, 237, 160-170, doi:10.1016/j.agrformet.2017.01.022.

Noormets, A., McNulty, S. G., Domec, J. C., Gavazzi, M., Sun, G., and King, J. S. (2012). The role of harvest residue in rotation cycle carbon balance in loblolly pine plantations. Respiration partitioning approach. Global Change Biology, 18(10), 3186-3201. 
Noormets, A., Gavazzi, M. J., McNulty, S. G., Domec, J. C., Sun, G. E., King, J. S., and Chen, J. (2010). Response of carbon fluxes to drought in a coastal plain loblolly pine forest. Global Change Biology, 16(1), 272-287.

Novick, K. A., Ficklin, D. L., Stoy, P. C., Williams, C. A., Bohrer, G., Oishi, A. C., ... and Scott, R. L. (2016). The increasing importance of atmospheric demand for ecosystem water and carbon fluxes. Nature Climate Change, 6(11), 1023-1027, doi:10.1038/nclimate3114.

Novick, K. A., Oren, R., Stoy, P. C., Siqueira, M. B. S., and Katul, G. G. (2009). Nocturnal evapotranspiration in eddy-covariance records from three co-located ecosystems in the Southeastern US: implications for annual fluxes. Agricultural and Forest Meteorology, 149(9), 1491-1504, doi:10.1016/j.agrformet.2009.04.005.

Novick, K. A., Stoy, P. C., Katul, G. G., Ellsworth, D. S., Siqueira, M. B. S., Juang, J., and Oren, R. (2004). Carbon dioxide and water vapor exchange in a warm temperate grassland. Oecologia, 138(2), 259-274.

Obeysekera, J., Browder, J., Hornung, L., and Harwell, M. A. (1999). The natural South Florida system I: Climate, geology, and hydrology. Urban Ecosystems, 3(3), 223-244.

Panda, U. C., Sundaray, S. K., Rath, P., Nayak, B. B., and Bhatta, D. (2006). Application of factor and cluster analysis for characterization of river and estuarine water systems - a case study: Mahanadi River (India). Journal of Hydrology, 331(3), 434-445.

Peichl, M., and Arain, M. A. (2007). Allometry and partitioning of above-and belowground tree biomass in an age-sequence of white pine forests. Forest Ecology and Management, 253(1), 68-80, doi:10.1016/j.foreco.2007.07.003. 
Peichl, M., Arain, M. A., and Brodeur, J. J. (2010). Age effects on carbon fluxes in temperate pine forests. Agricultural and Forest Meteorology, 150(7), 1090-1101, doi:10.1016/j.agrformet.2010.04.008.

Raz-Yaseef, N., Billesbach, D. P., Fischer, M. L., Biraud, S. C., Gunter, S. A., Bradford, J. A., and Torn, M. S. (2015). Vulnerability of crops and native grasses to summer drying in the US Southern Great Plains. Agriculture, Ecosystems and Environment, 213, 209-218, doi: 10.1061/j.agee.2015.07.021.

Ruehr, N. K., Martin, J. G., and Law, B. E. (2012). Effects of water availability on carbon and water exchange in a young ponderosa pine forest: Above-and belowground responses. Agricultural and Forest Meteorology, 164, 136-148.

Ryan, G. D., Sylvester, E. V., Shelp, B. J., and Newman, J. A. (2015). Towards an understanding of how phloem amino acid composition shapes elevated $\mathrm{CO}_{2}$-induced changes in aphid population dynamics. Ecological Entomology, 40(3), 247-257.

Saltelli, A., Ratto, M., Andres, T., Campolongo, F., Cariboni, J., Gatelli, D., ... and Tarantola, S. (2008). Global sensitivity analysis: the primer. New York, NY: John Wiley and Sons.

Schedlbauer, J. L., Oberbauer, S. F., Starr, G., and Jimenez, K. L. (2010). Seasonal differences in the $\mathrm{CO}_{2}$ exchange of a short-hydroperiod Florida Everglades marsh. Agricultural and Forest Meteorology, 150(7), 994-1006, doi:10.1016/j.agrformet.2010.03.005.

Schedlbauer, J. L., Munyon, J. W., Oberbauer, S. F., Gaiser, E. E., and Starr, G. (2012). Controls on ecosystem carbon dioxide exchange in short-and long-hydroperiod Florida Everglades freshwater marshes. Wetlands, 32(5), 801-812, doi:10.1007/s13157-012-0311-y. 
Schedlbauer, J. L., Oberbauer, S. F., Starr, G., and Jimenez, K. L. (2011). Controls on sensible heat and latent energy fluxes from a short-hydroperiod Florida Everglades marsh. Journal of Hydrology, 411(3), 331-341, doi: 10.1016/j.jhydrol.2011.10.014.

Scheffer, M., Carpenter, S., Foley, J. A., Folke, C., and Walker, B. (2001). Catastrophic shifts in ecosystems. Science, 413(6856), 591-596.

Schmidt, A., Hanson, C., Kathilankal, J., and Law, B. E. (2011). Classification and assessment of turbulent fluxes above ecosystems in North America with self-organizing feature map networks. Agricultural and Forest Meteorology, 151(4), 508-520, doi:10.1016/j.agrformet.2010.12.009.

Scott, R. L., Biederman, J. A., Hamerlynck, E. P., and Barron-Gafford, G. A. (2015). The carbon balance pivot point of southwestern US semiarid ecosystems: Insights from the 21st century drought. Journal of Geophysical Research: Biogeosciences, 120(12), 2612-2624.

Shi, H., Li, L., Eamus, D., Huete, A., Cleverly, J., Tian, X., ... and Rotenberg, E. (2017). Assessing the ability of MODIS EVI to estimate terrestrial ecosystem gross primary production of multiple land cover types. Ecological Indicators, 72, 153-164.

Shoemaker, W. B., Barr, J. G., Graham, S. L., and Botkin, D. B. (2015). Carbon exchange between the atmosphere and subtropical forested cypress and pine wetlands. Biogeosciences, 12(8), 2285-2300, doi:10.5194/bg-12-2285-2015.

Stoy, P. C., Mauder, M., Foken, T., Marcolla, B., Boegh, E., Ibrom, A., ... and Cescatti, A. (2013). A data-driven analysis of energy balance closure across FLUXNET research sites: The role of landscape scale heterogeneity. Agricultural and Forest Meteorology, 171, 137-152. 
Stoy, P. C., Richardson, A. D., Baldocchi, D. D., Katul, G. G., Stanovick, J., Mahecha, M. D., ... and Arriga, N. (2009). Biosphere-atmosphere exchange of $\mathrm{CO}_{2}$ in relation to climate: A cross-biome analysis across multiple time scales, Biogeosciences, 6, 2297-2312, doi:10.5194/bg-6-2297-2009.

Stoy, P. C., Katul, G. G., Siqueira, M. B., Juang, J. Y., McCarthy, H. R., Kim, H. S., ... and Oren, R. (2005). Variability in net ecosystem exchange from hourly to inter-annual time scales at adjacent pine and hardwood forests: a wavelet analysis. Tree Physiology, 25(7), 887902.

Suyker, A. E., Verma, S. B., Burba, G. G., and Arkebauer, T. J. (2005). Gross primary production and ecosystem respiration of irrigated maize and irrigated soybean during a growing season. Agricultural and Forest Meteorology, 131(3), 180-190.

Urbanski, S., Barford, C., Wofsy, S., Kucharik, C., Pyle, E., Budney, J., ... and Munger, J. W. (2007). Factors controlling $\mathrm{CO}_{2}$ exchange on timescales from hourly to decadal at Harvard Forest. Journal of Geophysical Research: Biogeosciences, 112, G02020, doi:02010.01029/02006JG000293.

Van der Molen, M. K., Dolman, A. J., Ciais, P., Eglin, T., Gobron, N., Law, B. E., ... and Chen, T. (2011). Drought and ecosystem carbon cycling. Agricultural and Forest Meteorology, 151(7), 765-773.

Vickers, D., Thomas, C., and Law, B. E. (2009). Random and systematic $\mathrm{CO}_{2}$ flux sampling errors for tower measurements over forests in the convective boundary layer. Agricultural and Forest Meteorology, 149(1), 73-83, doi:10.1016/j.agrformet.2008.07.005. 
Vickers, D., Thomas, C., Pettijohn, C., Martin, J. G., and Law, B. (2012). Five years of carbon fluxes and inherent water-use efficiency at two semi-arid pine forests with different disturbance histories. Tellus Series B: Chemical and Physical Meteorology, 64(1), 17159, doi: 10.3402/tellusb.v64i0.17159.

Wagle, P., Xiao, X., Gowda, P., Basara, J., Brunsell, N., Steiner, J., and Anup, K. C. (2017). Analysis and estimation of tallgrass prairie evapotranspiration in the central United States. Agricultural and Forest Meteorology, 232, 35-47, doi:10.1016/j.agrformet.2016.08.005.

Wagle, P., Xiao, X., Kolb, T. E., Law, B. E., Wharton, S., Monson, R. K., ... and Gowda, P. (2016). Differential responses of carbon and water vapor fluxes to climate among evergreen needleleaf forests in the USA. Ecological Processes, 5(1), 1-17.

Wei, L., Lockington, D. A., Poh, S. C., Gasparon, M., and Lovelock, C. E. (2013). Water use patterns of estuarine vegetation in a tidal creek system. Oecologia, 172(2), 485-494.

Wold, S., Sjöström, M., and Eriksson, L. (2001). PLS-regression: a basic tool of chemometrics. Chemometrics and Intelligent Laboratory Systems, 58(2), 109-130.

Wylie, B. K., Fosnight, E. A., Gilmanov, T. G., Frank, A. B., Morgan, J. A., Haferkamp, M. R., and Meyers, T. P. (2007). Adaptive data-driven models for estimating carbon fluxes in the Northern Great Plains. Remote Sensing of Environment, 106(4), 399-413.

Xiao, J., Chen, J., Davis, K. J., and Reichstein, M. (2012). Advances in upscaling of eddy covariance measurements of carbon and water fluxes. Journal of Geophysical Research: Biogeosciences, 117, G00J01. 
Xiao, J., Ollinger, S. V., Frolking, S., Hurtt, G. C., Hollinger, D. Y., Davis, K. J., ... and Baldocchi, D. D. (2014). Data-driven diagnostics of terrestrial carbon dynamics over North America. Agricultural and Forest Meteorology, 197, 142-157, doi:10.1016/j.agrformet.2014.06.013.

Xiao, J., Zhuang, Q., Baldocchi, D. D., Law, B. E., Richardson, A. D., Chen, J., ... and Verma, S. B. (2008). Estimation of net ecosystem carbon exchange for the conterminous United States by combining MODIS and AmeriFlux data. Agricultural and Forest Meteorology, 148(11), 1827-1847, doi:10.1016/j.agrformet.2008.06.015.

Xie, J., Chen, J., Sun, G., Chu, H., Noormets, A., Ouyang, Z., ... and Guan, W. (2014). Longterm variability and environmental control of the carbon cycle in an oak-dominated temperate forest. Forest Ecology and Management, 313, 319-328, doi: 10.1016/j.foreco.2013.10.032.

Yvon-Durocher, G., Caffrey, J. M., Cescatti, A., Dossena, M., del Giorgio, P., Gasol, J. M., ... and Woodward, G. (2012). Reconciling the temperature dependence of respiration across timescales and ecosystem types. Nature, 487(7408), 472.

Zhang, Y., Xiao, X., Zhou, S., Ciais, P., McCarthy, H., and Luo, Y. (2016). Canopy and physiological controls of GPP during drought and heat wave. Geophysical Research Letters, 43(7), 3325-3333.

Zhong, Q., Wang, K., Lai, Q., Zhang, C., Zheng, L., and Wang, J. (2016). Carbon dioxide fluxes and their environmental control in a reclaimed coastal wetland in the Yangtze Estuary. Estuaries and Coasts, 39(2), 344-362. 


\section{APPENDICES}

Figure A: Observed vs. predicted Z-scores of NEE for the 57 study sites
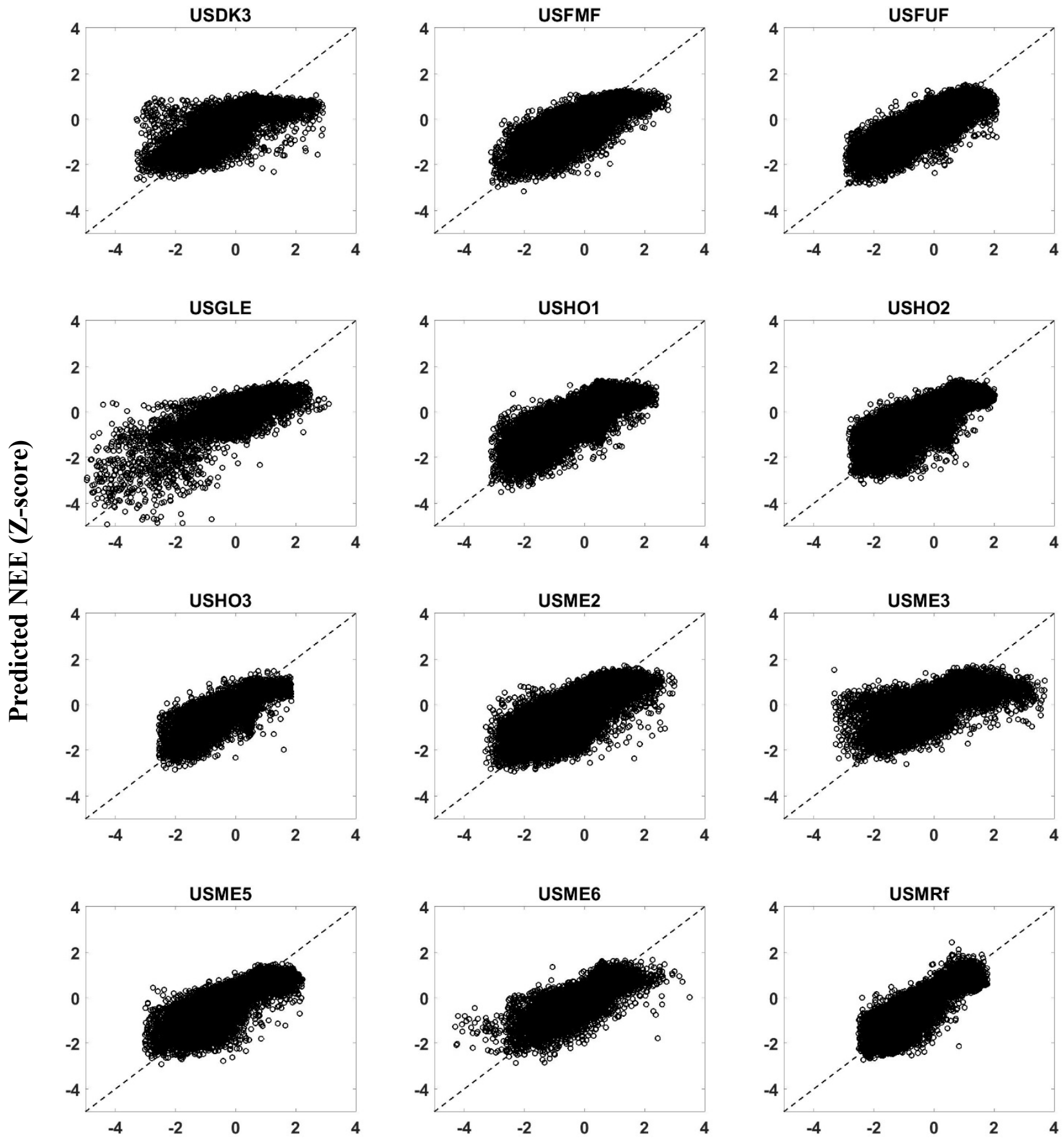

Observed NEE (Z-score) 
Figure A: continued
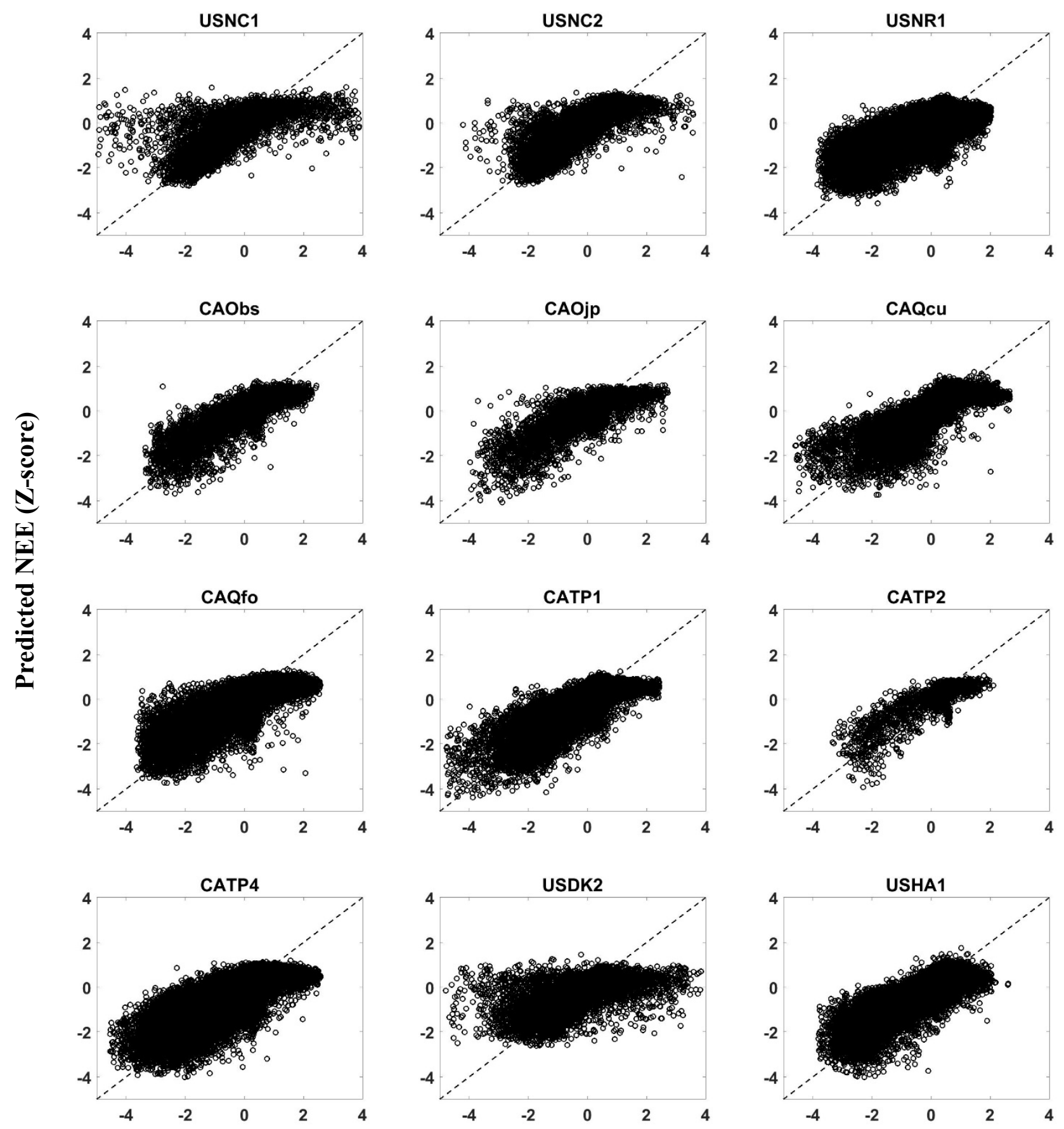

Observed NEE (Z-score) 
Figure A: continued
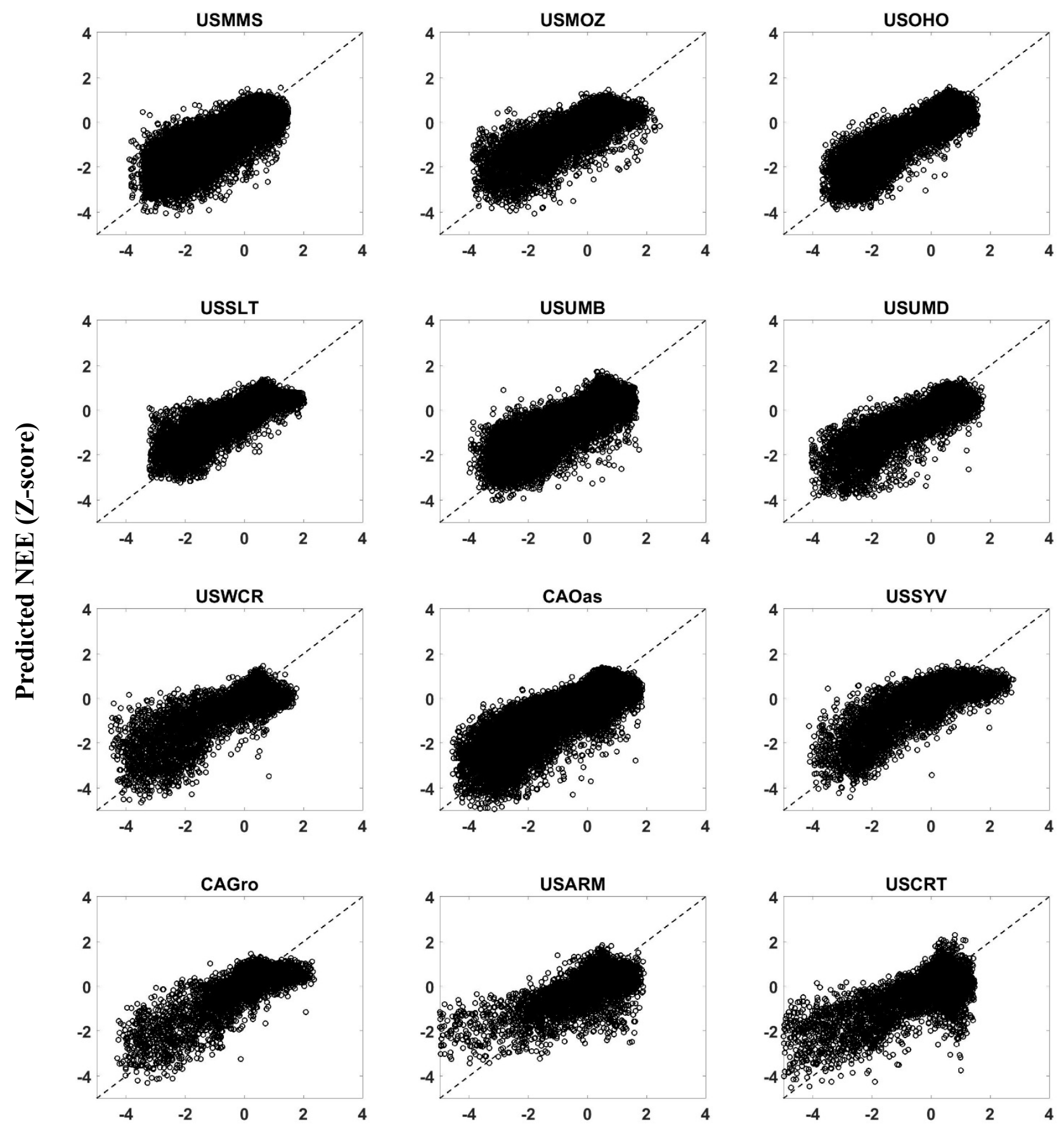

Observed NEE (Z-score) 
Figure A: continued
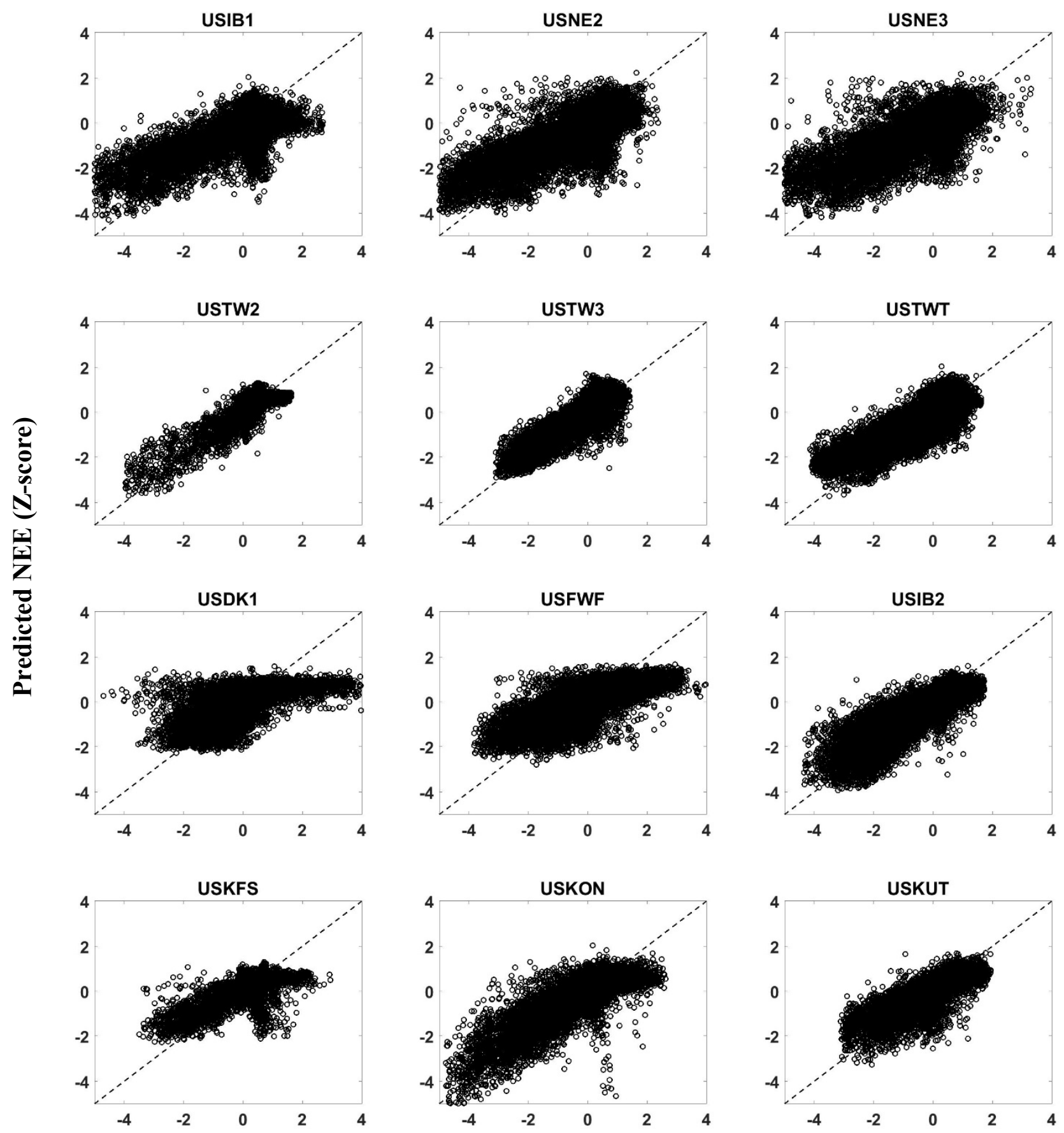

Observed NEE (Z-score) 
Figure A: continued
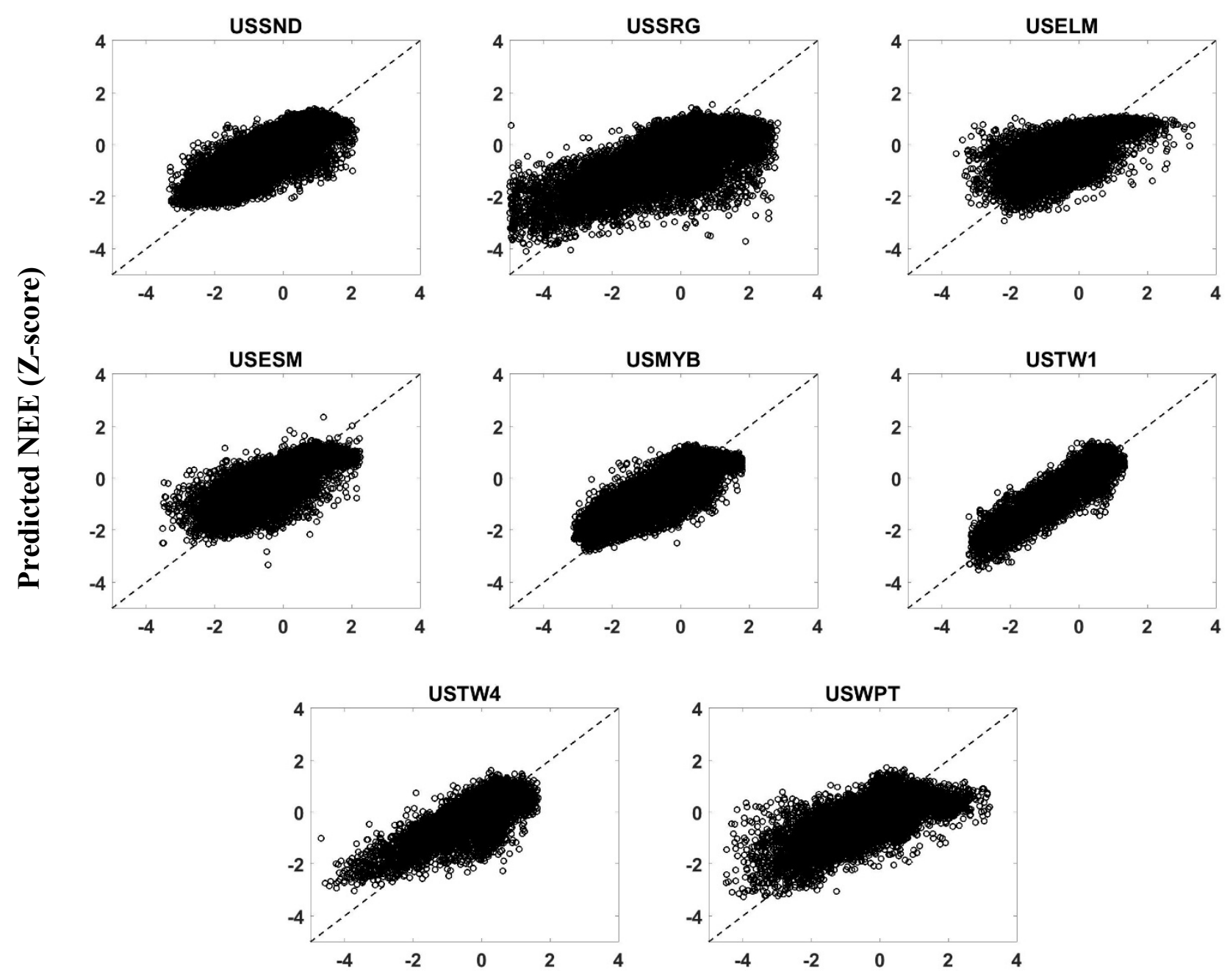

Observed NEE (Z-score) 